\title{
Constraints on Dark Matter interactions from structure formation: damping lengths ${ }^{\star}$
}

\author{
C. Boehm ${ }^{1,2}$ and R. Schaeffer ${ }^{3}$ \\ 1 Department of Physics, Theory division, CERN, 1211 Geneva 23, Switzerland \\ 2 LAPTH, 9 chemin de Bellevue, BP 110, 74941 Annecy-Le-Vieux Cedex, France \\ e-mail: Celine.Boehm@cern.ch \\ 3 SPhT, CEA Saclay, 91191 Gif-sur-Yvette, France \\ e-mail: Richard.Schaeffer@cea.fr
}

Received 22 October 2004 / Accepted 21 February 2005

\begin{abstract}
Weakly Interacting Massive Particles are often said to be the best Dark Matter candidates. Studies have shown that large Dark Matter-photon or Dark Matter-baryon interactions could be allowed by cosmology. Here we address the question of the role of the Dark Matter interactions in more detail to determine at which extent Dark Matter has to be necessarily weakly interacting. To this purpose, we compute the collisional damping (and free-streaming) scales of generic interacting Dark Matter candidates and investigate the effects on structure formation. Our calculations are valid provided the Dark Matter particles have experienced a phase of statistical equilibrium at some stage during their evolution. By comparing these damping lengths to the scale of the smallest primordial structures known to exist in the Universe, we obtain necessary conditions that any candidate must satisfy. These conditions are expressed in terms of the Dark Matter particles' mass and either the total Dark Matter interaction rate or the interaction rate of Dark Matter with a specific species. The case of Dark Matter interacting with neutrinos or photons is considered in full detail. Our results are valid even for energy dependent cross-sections and for any possible initial fluctuations spectrum. We point out the existence of new Dark Matter scenarios and exhibit new damping regimes. For example, an interacting candidate may bear a similar damping than that of collisionless Warm Dark Matter particles. The main difference is due to the Dark Matter coupling to interacting (or even freely-propagating) species. Our approach yields a general classification of Dark Matter candidates which extends the definitions of the usual Cold, Warm and Hot Dark Matter scenarios when interactions, weak or strong, are considered.
\end{abstract}

Key words. elementary particles - dark matter - large-scale structure of Universe

\section{Introduction}

The damping of primordial fluctuations has been considered more than 30 years ago (Silk 1968a,b; Misner 1968) when baryonic matter was popular. In the early eighties, when the existence of Dark Matter became a serious possibility, it was quickly understood that fluctuations made of Weakly Interacting Massive Particles will render galaxy formation much easier (Gunn et al. 1978; Peebles 1982; Bond \& Szalay 1983). If the Dark Matter interactions are weak enough and the Dark Matter mass heavier than a few $\mathrm{keV}$, one indeed expects no collisional damping effects, and no free-streaming on scales relevant for galaxy formation. Such particles (e.g. neutralinos) are now embedded in the well-known Cold Dark Matter (CDM) scenario. As a limiting case, particles which marginally comply with the free-streaming constraints are usually referred to as Warm Dark Matter (WDM) candidates, the archetype being (Davis et al. 1981; Blumenthal et al. 1982) particles with a mass of $\sim 1 \mathrm{keV}$ whose damping length is about $100 \mathrm{kpc}$.

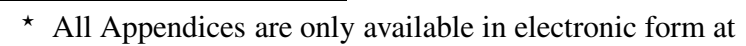
http://www. edpsciences.org
The CDM initial conditions reproduce rather well the mass distribution of the observed large scale structures. This scenario however is in danger to yield an excess of small galaxies (Schaeffer \& Silk 1985; Moore et al. 1998; Moore 1994). It also has difficulties to reproduce the shape of the galaxy rotation curves at the smaller scales (Moore et al. 1999).

The WDM model, known to reproduce better the observed faint end of the galaxy multiplicity function (Schaeffer \& Silk 1988), was revived (e.g. Sommer-Larsen \& Dolgov 1999) as a possible solution to the discrepancy between observations and CDM numerical simulations. However, the collisionless WDM scenario does not seem to be the solution to all discrepancies (Knebe et al. 2002; Barkana et al. 2001), especially if one considers the constraints from the late reionization signal claimed to be found by WMAP (Spergel et al. 2003; Yoshida et al. 2003).

Some of the potential problems of the CDM scenario may possibly be alleviated by invoking astrophysical processes (e.g. Chiu et al. 2001; Stoehr et al. 2002; Dekel \& Devor 2003) or perhaps by redoing data analysis of the dwarf and Low Surface Brightness rotation curves (e.g. van den Bosch \& Swaters 2001; van den Bosch et al. 1999). Also, the Dark halo profile of 
the Milky Way may (Prada et al. 2004) be more cuspy than previously found (Binney \& Evans 2001). Should the still remaining discrepancy between numerical simulations and observations be due to the nature of Dark Matter or not, a thorough investigation of the Dark Matter properties seems worthwhile.

In particular, one may wonder whether taking into account the Dark Matter interactions - generally considered to be negligible - would help to solve the Dark Matter crisis at small scales.

This is discussed by Spergel \& Steinhardt (2000) who consider strongly self-interacting Dark Matter to comply with the shape of the galaxy rotation curves (Moore et al. 1999). However, according to further investigations (Yoshida et al. 2000; Bautz et al. 2002), this suggestion, at least in its original form, seems not to be the answer.

One can then address a more general question to determine which kind of interaction is allowed for Dark Matter, with which particles, and with which strength. The physical process that we will consider in this paper is the damping of primordial fluctuations. At late stages of their evolution, due to the gravitational instability, the fluctuations evolve from the linear to the non-linear regime. Up to the present epoch, there is an amplification of about 5 orders of magnitude. We know from the microwave background that the primordial amplitudes barely exceeds the $10^{-5}$ level. Any fluctuation of smaller amplitude will remain in the linear regime. In the primordial universe, the fluctuations are generically stable and oscillating but their original amplitude may be drastically reduced by viscous effects such as shear, heat and bulk viscosity, or simply by the mixing of fluids. The associated damping affects drastically the initial amplitude below a well-defined spatial scale, the damping scale. Damping must be avoided at the scales where condensed primordial objects are known to exist. This provides an observational limit for the damping scale and, in turn, for the particles' physical properties (velocities, interaction rates) which determine the efficiency of this damping.

The original move, which led to the introduction of weakly interacting Dark Matter particles (that are now embedded in the Cold Dark Matter scenario), was to avoid Silk damping as well as prohibitive free-streaming. In the present paper, we extend this approach to other Dark Matter interactions, say interactions of Dark Matter with any other species, including itself and seek constraints on the allowed Dark Matter particles' parameter space. First insights were given in Boehm et al. (2001, 2002); Chen et al. (2002). We undertake an exhaustive and extensive analysis by considering a classification which allows to discuss the possible interactions in the medium whatever the nature of the Dark Matter provided the latter underwent a phase in which it was in thermodynamical equilibrium. To make contact with the usual classification of Dark Matter scenarios, we discuss also additional aspects, in particular the way the Dark Matter particles achieve their relic density.

We start by relaxing the assumption of collisionless Dark Matter to estimate its collisional and free-streaming damping effects. By imposing that these two damping mechanisms do not prevent the formation of observed structures, we can define the range of Dark Matter mass and interaction rates that are compatible with observations. This is independent of any particle physics. We then classify all generic Dark Matter candidates in a 2-dimensional parameter space (interaction rate versus mass) and determine what regions of this parameter space are allowed by the present observations.

Our calculations are valid for massive neutrinos, provided their mass is within the known experimental and observational limits. For the sake of comparison with the general case we treat here, we use at various places a "reference", the cosmology in which i) the radiation has two components (namely photons and massless neutrinos, both having the standard temperature); and ii) the matter is made of two species, the baryons and the Dark Matter.

As pointed out in Boehm et al. (2001), the collisional damping scale of the Dark Matter fluctuations can be expressed in terms of two separate contributions, namely the self-damping and induced-damping contributions.

The self-damping is related to the collisional propagation of the Dark Matter particles. It may be directly compared with their free-streaming scale.

The induced-damping corresponds to the (collisional or free-streaming) damping acquired by a species $i$ and transmitted to the Dark Matter fluctuations as long as the coupling between Dark Matter and this species is large enough. The induced-damping effect can be seen as a generalization of the Silk damping (of baryons coupled to photons), here applied to the case of Dark Matter particles coupled to any possible species $i$. We especially focus our attention on the coupling to neutrinos and photons, seen to provide a quite large damping and yield rather stringent constraints.

Our results are given in two separate papers. The present one (referred to as Paper I) is dedicated to the calculation of the free-streaming and collisional damping scales associated with any interacting Dark Matter particles. We then derive the mass and interaction rates of the Dark Matter candidates allowed by structure formation. This is a rather technical paper. The second one (Paper II), deals with the physical and astrophysical relevance of the candidates whose damping scales, calculated here, are not prohibitive. These two papers have been written in a self-contained way and can be read independently.

The present paper is organized as follows: in Sect. 2, we recall the expressions of the different transport coefficients for an imperfect fluid made of several species (relativistic or not), and take this opportunity to discuss issues for which there is still some confusion in the litterature. In Sect. 3, we derive the expressions of the self-damping and induced-damping lengths. We also point out the existence of a new damping effect, that we call mixed-damping. The latter is a special case of induced damping. It describes the damping acquired by Dark Matter particles that would be coupled to a species which is free-streaming. In Sect. 4, we derive the constraints on the Dark Matter properties from the calculation of the freestreaming and self-damping scales. For the specific case of Dark Matter coupled to neutrinos or photons, the calculation of the induced-damping lengths and the derivation of the associated constraints on interaction rates and cross-sections are done in Sects. 5 and 6 respectively.

The definitions of the quantities used in this paper are given in Appendix A. We derive in Appendix B expressions 
for the evolution of the Dark Matter density, slightly simplified to be systematically used in our analytical calculations. The interaction rates appearing in the present work are given in the same appendix. The regions of the Dark Matter parameter space (Dark Matter particles' mass and interaction rates) where the various damping lengths take different forms are explicitly given in Appendix C. Finally, in Appendix D, we display analytical expressions for the damping scales and for the associated limits on the Dark Matter interaction rates and crosssections.

\section{Transport coefficients}

To study the collisional damping of Dark Matter fluctuations, we first need to determine the transport coefficients of an imperfect fluid in statistical ${ }^{1}$ equilibrium. The relevant coefficients are the shear viscosity, the heat conduction and the bulk viscosity. They appear in the expression of the energy-momentum tensor of an imperfect fluid, see e.g. Weinberg (1971).

Typically, we are lead to evaluate the transport coefficients of a mixture made of several species $i$ (including Dark Matter). The key which will enable us to obtain constraints resulting from these transport coefficient on any kind of Dark Matter will be to write these transport coefficients common to all species in the mixture as a sum over specific contributions. The latter may be viewed as the share of each of the species, contributing to the viscosity of the whole fluid ${ }^{2}$. The requirement, in a second stage, that each of the contribution in the sum, separately, must not be the source of prohibitive damping is then much simpler and will yield necessary conditions.

Let us now derive the transport coefficients. The procedure for the shear viscosity was outlined in detail by Chapman $\&$ Cowling (1970) for a mixture of non-relativistic particles. Here, we shall develop this approach further to describe a mixture made of both relativistic and non-relativistic particles. This problem has not been addressed in the desired form in the literature. Similarly, we will also need to derive the suitable form for the heat conduction and bulk viscosity coefficients.

\subsection{Shear viscosity}

\subsubsection{Shear viscosity for a mixture of relativistic and non-relativistic species}

Let us start with the expression given by Chapman \& Cowling (1970) which is valid for a composite fluid made of nonrelativistic species $i$ in statistical equilibrium. We rewrite it here

\footnotetext{
${ }^{1}$ We use the word thermal equilibrium for particles whose temperature and velocities equilibrate at least locally, but which are not in chemical equilibrium. Thermodynamical equilibrium is meant for particles in both thermal as well as chemical equilibrium. Statistical equilibrium stands for particles either in thermal or in thermodynamical equilibrium.

${ }^{2}$ It is worth emphasizing that the statistical equilibrium of the fluid ensures that each of the species experiences the same transport coefficients, given by the sum of all contributions.
}

in a more convenient way to make the generalization to the relativistic case easier:

$\eta=\sum_{i} \eta_{i}$

whith an individual contribution $\eta_{i}$ of each species $i$

$\eta_{i}=p_{i} / \Gamma_{i}$

where

$\Gamma_{i}=\sum_{j} \Gamma_{i j}$

represents the total interaction rate of species $i$. The specific interaction rate of species $i$ with species $j$ will be written as

$\Gamma_{i j}=\langle\overline{\sigma v}\rangle_{i j} n_{j}$

where $n_{j}$ is the number density of the species $j$ and $\langle\overline{\sigma v}\rangle_{i j}$ is the suitably weighted statistical average of the interaction crosssection between $i$ and $j$. Under this form, it seems natural to expect that this expression can be extended to the case of relativistic particles. Therefore one can write

$\eta=\sum_{i} \frac{p_{i}}{\Gamma_{i}}$

where the sum over $i$ runs over all the species in statistical equilibrium with the fluid. This form depends only on the pressure and on the interaction rate. Therefore, it may be expected to hold also for a fluid of relativistic particles. We thus expect the shear viscosity coefficient of a fluid made of relativistic and non-relativistic species in statistical equilibrium to be given by (5). The pressure $p_{i}$ may be written as $p_{i}=\frac{1}{3} v_{i}^{2} \rho_{i}$, where $v_{i}$ is the particle's rms velocity in units of $c$, so Eq. (5) equivalently reads

$\eta=\sum_{i} \frac{\rho_{i} v_{i}^{2}}{3 \Gamma_{i}}$

\subsubsection{Derivation}

The simplest way to convince oneself that (5) or (6) are the relevant expressions is to use kinetic theory.

The transport of momentum is induced by a gradient in the collective fluid velocity $\boldsymbol{V}$ carried by all species of the fluid. The momentum flux induced by this transport of momentum may be written $\eta \nabla \boldsymbol{V}$, and corresponds to a term $\eta\left(\partial V^{a} / \partial x_{b}+\right.$ $\left.\partial V^{b} / \partial x_{a}-\frac{2}{3} \delta_{a b} \operatorname{div} \boldsymbol{V}\right)$ in the spatial part $T^{a b}$ of the energymomentum tensor.

Each species $i$ is expected to contribute its own share to the total transport of momentum, which we denote as $\eta_{i} \nabla \boldsymbol{V}$. We may derive the quantity $\eta_{i}$ by considering the momentum flux carried by each of the microscopic particles of species $i$. This momentum flux is $\boldsymbol{v} \boldsymbol{V}$ where $\boldsymbol{v}=\boldsymbol{q} / e, \boldsymbol{q}$ is the particle's velocity, $\boldsymbol{q}$ the particle's momentum and $e$ its energy: each particle of species $i$ hence carries the collective momentum $\boldsymbol{e} \boldsymbol{V}$. It is transported over a distance of the order of the particle's mean-free-path $\mathrm{v} / \Gamma_{i}$, in the direction $v$, inducing a net transport $\boldsymbol{v} \boldsymbol{V}(\boldsymbol{x})-\boldsymbol{v} e \boldsymbol{V}\left(\boldsymbol{x}-\boldsymbol{v} / \Gamma_{i}\right)$. The latter may be rewritten as 
$\frac{q^{2}}{3 e} / \Gamma_{i} \nabla \boldsymbol{V}$, taking advantage of the smallness of the mean-freepath and the isotropy of the momenta. After summing over all momenta of particles of a species $i$, the flux due to a gradient in $\boldsymbol{V}$ is then seen to be $p_{i} / \Gamma_{i} \nabla \boldsymbol{V}$, which translates into $p_{i} / \Gamma_{i}\left(\partial V^{a} / \partial x_{b}+\partial V^{b} / \partial x_{a}-\frac{2}{3} \delta_{a b} \operatorname{div} V\right)$ in $T^{a b}$. The coefficient $\eta_{i}$ associated with species $i$ is thus given by $\eta_{i}=p_{i} / \Gamma_{i}$. This is similar to the usual result for the shear viscosity of a single species $i$. The interaction rate $\Gamma_{i}$, however, is the total interaction rate, i.e. it includes all possible interactions with other species present in the composite fluid. Since the latter arguments do not depend on whether species $i$ is relativistic or not, the result holds for both relativistic and non-relativistic species.

The total momentum transported by all species in the composite fluid is the sum of the amount of momentum transported by each species. Therefore, the shear viscosity coefficient of a composite fluid is given by $\eta=\sum_{i} \eta_{i}$, which justifies Eqs. (5) and (6).

\subsection{Heat conduction}

One can also derive the expression of the heat conduction coefficient for a composite fluid (including relativistic and nonrelativistic species) by using kinetic theory. The expression of this coefficient for non-relativistic particles has not been given in the appropriate form by Chapman and Cowling. However, they mentioned that it should be similar to the one obtained for the shear viscosity coefficient.

A given species $i$ contributes to the transport of energy induced by a gradient of temperature through its own conduction coefficient $\lambda_{i}$. The latter is proportional to the particle's meanfree-path $v_{i} / \Gamma_{i}$, times a flux factor $v_{i} \partial \rho_{i} / \partial T$. The difference with the previous case (2.1) is that, here, we consider the energy flux due to a change of temperature. Specifically, we get $^{3}$

$\lambda_{i} T=\frac{\rho_{i} v_{i}^{2}}{3 \Gamma_{i}} \frac{\partial \ln \rho_{i}}{\partial \ln T}$,

which holds whether the fluid is relativistic or not. The total energy transported by all species is the sum of the individual contributions. Yet, the heat conduction coefficient of a composite fluid is given by $\lambda=\sum_{i} \lambda_{i}$. This finally yields

$\lambda T=\sum_{i} \frac{\rho_{i} v_{i}^{2}}{3 \Gamma_{i}} \frac{\partial \ln \rho_{i}}{\partial \ln T}$.

\subsection{Bulk viscosity}

The bulk viscosity is related to departures from equilibrium induced by a non-zero divergence of the velocity field. The latter induces a compression or expansion of the fluid elements which may result in local conditions differing from the global ones. The former have to be compensated to recover the general statistical equilibrium conditions. This required adjustment provides for another source of viscosity.

Departures from chemical equilibrium also induce bulk viscosity contributions. The latter have already been applied to

\footnotetext{
3 The derivatives with respect of the temperature are to be taken at fixed volume.
}

other, specific, problems (e.g. Haensel \& Schaeffer 1992), and may be included by using similar techniques. They allow to constrain the chemical reaction rates, in addition to the collision rates we consider in the present paper.

We consider in detail below the departures from thermal equilibrium, specifically. All the conditions that we obtain in this paper on the collision rates thus are necessary conditions. They must be satisfied separately, keeping in mind that there may exist additional constraints on the chemical reaction rates.

It is worth to note, at this stage, that our calculation of the bulk viscosity due to departures from thermal equilibrium turns out to go much beyond what may be found in the existing literature.

\subsubsection{Single fluid}

A straightforward resolution of the transport equations, as outlined in Bernstein (1988), yields for a single species $i$

$$
\zeta_{i}^{(\text {single })}=\frac{\partial \rho_{i} / \partial \ln T}{\Gamma_{i}}\left[\omega_{i}-\left(\frac{\partial p_{i} / \partial \ln T}{\partial \rho_{i} / \partial \ln T}\right)^{2}\right]
$$

The rate $\Gamma_{i}$ is the collision rate for particles of species $i$ with themselves. The coefficient $\omega_{i}$ is a statistical average over momentum-dependent functions, describing how the stretching of the momenta by the expansion (or compression) directly affects pressure and density while the ratio $\frac{\partial p_{i} / \partial \ln T}{\partial \rho_{i} / \partial \ln T}$ describes how the final equilibrium distribution (i.e. the temperature) is affected. For a single fluid, the source of bulk viscosity is therefore the offset between the expansion (or compression) which affects the momenta producing a non-equilibrium distribution and statistical equilibrium which tries to cope with this change by readjusting the temperature. The Boltzmann equilibrium distribution turns out to be preserved for an ultrarelativistic fluid (the momentum stretching $q \rightarrow q / a$ inducing exactly $T \rightarrow T / a)$ as well as for a non relativistic fluid of conserved particles $\left(q \rightarrow q / a\right.$ inducing exactly $\left.T \rightarrow T / a^{2}\right)$, so the bulk viscosity vanishes in both cases. In intermediate situations, this is not so: the deviations of $\omega_{i}$ from $\frac{\partial p_{i} / \partial \ln T}{\partial \rho_{i} / \partial \ln T}$ however are quite small. A numerical estimate shows that $\zeta_{i}^{\text {(single) }} / \eta_{i}$ in general barely exceeds the $1 \%$ level. This ratio however is sizable for the case of zero chemical potential annihilating massive particles, for instance $\zeta_{i}^{\text {(single) }} / \eta_{i} \sim 0.5$ at $T \sim m / 20$, reaching asymptotically $2 / 3$ for the (unrealistic: it corresponds to a number density $n_{i} \propto \mathrm{e}^{-m / T} \rightarrow 0$ ) case $m / T \rightarrow \infty$.

The result (9) for a single fluid is consistent with the result of Bernstein (1988). However, the latter author considered only massive particles in the limit $m / T \ll 1$, that is ultra relativistic particles. The contribution (9) does not exist in the approach of Weinberg (1971) who considered either strictly massless or massive non relativistic, stable $(m / T \gg 1)$ particles. In the latter two cases, indeed, the contribution (9) vanishes. As we shall see in the next section, there is additional bulk viscosity generated in a composite fluid, which is the question addressed by Weinberg. Despite the comparison made by Bernstein (1988) between his result and the one obtained by Weinberg (1971), exhibiting some alleged agreement between the two, these 
authors turn out to describe different physical phenomena, with no overlap of their results.

\subsubsection{Composite fluid}

For a composite fluid, the total bulk viscosity is not simply the sum of Eq. (9) over all species $i$ which are coupled together. There is an additional source of inelasticity (compared to the single fluid case) which originates from the constraint that each of the components $i$ should acquire the general temperature of the medium rather than the one it would acquire were it isolated. This implies a rearrangement of the equilibrium distribution directly related to exchanges among the various species of the fluid. This is in contrast with the transport of collective motion or temperature, for which each species contributes independently.

This calculation was first done by Weinberg (1971). The latter is enlightened by an interesting discussion due to Zimdahl (1996).

With the assumption that the species $i$ are in statistical equilibrium, one gets, for a composite fluid:

$\zeta=\delta \rho\left[\omega-\left(\frac{\delta p}{\delta \rho}\right)^{2}\right]$

with

$\delta \rho=\sum_{i} \frac{\partial \rho_{i} / \partial \ln T}{\Gamma_{i}}$

$\delta p=\sum_{i} \frac{\partial p_{i} / \partial \ln T}{\Gamma_{i}}$

and

$\omega=\sum_{i} \frac{\partial \rho_{i} / \partial \ln T}{\Gamma_{i}} \omega_{i} / \sum_{i} \frac{\partial \rho_{i} / \partial \ln T}{\Gamma_{i}}$.

The weighting by the inverse of the interaction rates (i.e. by the collision time of each species $i$ interacting with all species within the fluid) is new. It arises from the actual resolution of the transport equations in the relaxation time approximation, the key being to use different collision times for each species (this is the very same approximation as in the previous cases for the shear viscosity and heat conduction) and to account for energy conservation.

The expression (10) may be rearranged as

$\zeta=\sum_{i} \zeta_{i}$

with

$$
\begin{aligned}
\zeta_{i}= & \frac{\partial \rho_{i} / \partial \ln T}{\Gamma_{i}}\left[\omega_{i}-\left(\frac{\partial p_{i} / \partial \ln T}{\partial \rho_{i} / \partial \ln T}\right)^{2}\right] \\
& +\frac{\partial \rho_{i} / \partial \ln T}{\Gamma_{i}}\left(\frac{\partial p_{i} / \partial \ln T}{\partial \rho_{i} / \partial \ln T}-\frac{\delta p}{\delta \rho}\right)^{2} .
\end{aligned}
$$

The first term corresponds to the bulk viscosity of a single fluid due to the fact the temperature of species $i$ has to adjust to any expansion/compression, which is a constraint when particles are neither fully ultra-relativistic nor purely non relativistic. It differs however from (9) by the interaction rate $\Gamma_{i}$ which is now the collision rate of particles of species $i$ with all the species in the composite fluid (and not only with themselves). Indeed, all collisions contribute to the adjustment of the temperature.

The second term of Eq. (12) is a contribution specific to a composite fluid:

$\zeta_{i}^{\text {(composite) }} \sim \frac{\partial \rho_{i} / \partial \ln T}{\Gamma_{i}}\left(\frac{\partial p_{i} / \partial \ln T}{\partial \rho_{i} / \partial \ln T}-\frac{\delta p}{\delta \rho}\right)^{2}$

It is due to the fact that the temperature of species $i$ also has to adjust to the change of the general temperature of the medium under the expansion/compression. Such a change represents another constraint when the equation of state of the whole medium and the one of species $i$ are not the same, more specifically when $\frac{\partial p_{i} / \partial \ln T}{\partial \rho_{i} / \partial \ln T} \neq \frac{\delta p}{\delta \rho}$. Indeed, the contribution (13) may be viewed as the pressure offset due to a departure from equilibrium, by $\Delta f_{i}=\Delta T_{i} \partial f_{i} / \partial T$, of the Boltzmann distribution $f_{i}$ of species $i$, in-between two collisions, with $\Delta T_{i}=\frac{1}{\Gamma_{i}}\left(\frac{\partial p_{i} / \partial \ln T}{\partial p_{i} / \partial \ln T}-\frac{\delta p}{\delta \rho}\right) \operatorname{div} \boldsymbol{V}$. Note that this form induces no offset in the total energy-density, as required by energy conservation ${ }^{4}$.

The form (13) is in agreement with the expression derived by Zimdahl (1996). However, our result is obtained under the simple and unique assumption that all species are coupled and in statistical equilibrium. We also take into account, in accordance with the style of the present paper but to contrast with previous work, that the coupling of each species $i$ with the whole medium is, as a rule, different. On the other hand, Zimdahl (1996) makes much more restrictive assumptions, since they are sufficient to make his point. He considers species $i$ which are already in equilibrium with themselves at different internal temperatures $T_{i}$, under processes he does not show explicitly, and uses for his actual calculation a unique interaction rate for the various species just to equilibrate their temperature.

In his paper, Zimdahl (1996) noted that his more elaborate expression differs from the result derived from Weinberg (1971) under the same assumptions, but did not pin down explicitly which step in the latter approach was at the origin of the difference.

Our form (13) readily shows that the contribution of the photons $(i=\gamma)$ to the sum (11) specifically is the (sole) term retained by Weinberg (1971). It is implicit in the latter work that there is a second contribution due to the matter component $(i=m)$ which however was omitted on grounds the matter interaction rate ( $\Gamma_{\mathrm{m}}$ in our notation $)$ is assumed to be very large. But due to cancellations in the square of Eq. (13), the pre-factor does not determine the size of the contribution. Indeed, this omitted second contribution is in most cases the dominant one. This is the explanation for the difference between these two authors, and will be discussed in Sect. 2.3.3 using the same composite fluid these authors have considered.

${ }^{4}$ This can be explicitly checked: $\Delta \rho=\sum_{i} \int \mathrm{d}^{3} p_{i} e_{i} \Delta f_{i}=$ $\sum_{i} \Delta T_{i} / T \partial \rho_{i} / \partial \ln T$ indeed vanishes due to the weighting, originally required to this purpose, by the factors $1 / \Gamma_{i}$. 
The contribution (13) to the bulk viscosity vanishes for a single fluid. So, as a rule, in Eq. (13), when the coefficient $\frac{\partial \rho_{i} / \partial \ln T}{\Gamma_{i}}$ in front of the bracket is large, the two terms within the bracket nearly cancel each other since the composite fluid is dominated by the single component $i$. Conversely, when the bracket is of order unity, it is necessarily for a subdominant species for which there is no cancellation. So, $\zeta$ in general never gets very large as compared to $\eta$. This is discussed in Sect. 2.3.3 for the generic example of a composite fluid made of matter and radiation. In all cases, we find $\zeta \leq \frac{4}{3} \eta$, if not $\zeta \ll \frac{4}{3} \eta$. As we will see below, in Sect. 3 , the viscosity terms enter the damping expression with a weight $\zeta+\frac{4}{3} \eta$. For the sake of the present study, we simply conclude that the bulk viscosity will not induce any new constraint as compared to the shear viscosity, a property which has been already used in Boehm et al. (2001). So, we do not need to consider the bulk viscosity for the limits on the interaction rates we seek.

These results are seen to be far more general than what is needed in the present paper and more accurate than the ones which may be found in the literature. We give indeed the expression of the bulk viscosity for a composite fluid as a sum of several well-identified contributions, due to different physical phenomena. This sets into perspective the various calculations done by the different authors. Also, we take into account the difference between the collision rates of various species, a move that is required by our aim to establish limits on these rates. We point out several inaccuracies in the literature. Assuming an average interaction rate, as done in previous work, leaves the latter conclusions unchanged. Needless to say, our findings are in agreement with the statements made in Boehm et al. (2001).

\subsubsection{Mixture of matter and radiation}

As an illustration of the above results, we discuss here explicitly the bulk viscosity of a composite fluid made of radiation (massless, relativistic, particles with zero chemical potential and a unique collision rate $\Gamma_{\mathrm{r}}$ ) and matter (non relativistic particles with a unique rate $\Gamma_{\mathrm{m}}$ ), assumed to form a unique fluid in statistical equilibrium. We discuss the various relevant cases, and provide the explanation for the different, and contradictory, results found in the literature. We hope this will clear out the matters.

The bulk viscosity for the composite fluid is the sum

$$
\zeta=\zeta_{\mathrm{r}}+\zeta_{\mathrm{m}}
$$

and has the same value for the matter as well as the radiation. Note that - although $\zeta_{\mathrm{r}}$ is the contribution to the bulk viscosity from the relativistic species - the bulk viscosity coefficient of the relativistic species is not $\zeta_{\mathrm{r}}$ but $\zeta$. Similarly, the contribution of the non-relativistic species to the bulk viscosity is $\zeta_{\mathrm{m}}$, but its bulk viscosity of course also is $\zeta$, the two species being part of the same fluid.

This bulk viscosity may be usefully compared to the shear viscosity of the composite fluid:

$\eta=\eta_{\mathrm{r}}+\eta_{\mathrm{m}}$, related to the pressure $p_{\mathrm{r}}$ of the radiation and $p_{\mathrm{m}}$ of the matter by $\eta_{\mathrm{r}}=p_{\mathrm{r}} / \Gamma_{\mathrm{r}}$ and $\eta_{\mathrm{m}}=p_{\mathrm{m}} / \Gamma_{\mathrm{m}}$.

The case of conserved matter particles. We first consider the case of stable matter particles, with a conserved particle number. There is then a negligible contribution of the form (9), which we omit. From Eq. (13), we get

$$
\begin{aligned}
\zeta_{\mathrm{r}} & =\frac{\partial \rho_{\mathrm{r}} / \partial \ln T}{\Gamma_{\mathrm{r}}}\left(\frac{1}{3}-\frac{\delta p}{\delta \rho}\right)^{2} \\
& =\frac{4}{3} \eta_{\mathrm{r}}\left(\frac{\eta_{\mathrm{m}} / 8}{\eta_{\mathrm{r}}+\eta_{\mathrm{m}} / 8}\right)^{2}
\end{aligned}
$$

and

$$
\begin{aligned}
\zeta_{\mathrm{m}} & =\frac{\partial \rho_{\mathrm{m}} / \partial \ln T}{\Gamma_{\mathrm{m}}}\left(\frac{2}{3}-\frac{\delta p}{\delta \rho}\right)^{2} \\
& =\frac{4}{3} \eta_{\mathrm{m}} / 8\left(\frac{\eta_{\mathrm{r}}}{\eta_{\mathrm{r}}+\eta_{\mathrm{m}} / 8}\right)^{2}
\end{aligned}
$$

since

$\delta \rho=12 \eta_{\mathrm{r}}+\frac{3}{2} \eta_{\mathrm{m}}$,

$\delta p=4 \eta_{\mathrm{r}}+\eta_{\mathrm{m}}$.

This yields

$\zeta=\frac{4}{3} \frac{\eta_{\mathrm{r}} \eta_{\mathrm{m}} / 8}{\eta_{\mathrm{r}}+\eta_{\mathrm{m}} / 8}$

For $\eta_{\mathrm{r}} \gg \eta_{\mathrm{m}}$ we have $\zeta_{\mathrm{r}} \sim \eta_{\mathrm{m}}^{2} / 48 \eta_{\mathrm{r}}\left(\ll \eta_{\mathrm{m}}\right) \ll \eta_{\mathrm{r}}$ and $\zeta_{\mathrm{m}} \sim$ $\eta_{\mathrm{m}} / 6<\eta_{\mathrm{m}}$. One can see in this example that, when the bound $\zeta_{i} \sim \eta_{i}$ is close to be reached, it is for a subdominant species. Indeed, we have $\zeta \propto \eta_{\mathrm{m}} \ll \eta \sim \eta_{\mathrm{r}}$. For the converse, somewhat less realistic assumption $\eta_{\mathrm{r}} \ll \eta_{\mathrm{m}}$, we would get $\zeta \sim \zeta_{\mathrm{r}} \propto \eta_{\mathrm{r}} \ll$ $\eta \sim \eta_{\mathrm{m}}$. This implies in particular $\zeta \ll \frac{4}{3} \eta$. The bulk viscosity is, as a rule, much smaller than the shear viscosity.

Annihilating matter. Here we consider the case of annihilating matter, when the chemical potential of the massive particles also vanishes. More specifically we consider the limit of large $m / T$ (having in mind $m / T \sim 20$ ) where $m$ is the mass of the annihilating particles and $T$ their temperature.

There is in this case a sizeable contribution of the form (9) to $\zeta_{\mathrm{m}}$ as discussed in the corresponding section:

$\zeta_{\mathrm{m}} \leq \frac{2}{3} \eta_{\mathrm{m}}$

Were the matter not coupled to radiation, this would be the only contribution to the bulk viscosity, with then $\zeta<\frac{4}{3} \eta$ since $\zeta=\zeta_{\mathrm{m}}$ and $\eta=\eta_{\mathrm{m}}$ in this case.

For matter coupled to radiation, there is also a contribution of the form (13) specific to a composite fluid. We get, with $b=\frac{m^{2}}{12 T^{2}}$,

$$
\begin{aligned}
\zeta_{\mathrm{r}} & =\frac{\partial \rho_{\mathrm{r}} / \partial \ln T}{\Gamma_{\mathrm{r}}}\left(\frac{1}{3}-\frac{\delta p}{\delta \rho}\right)^{2} \\
& =\frac{4}{3} \eta_{\mathrm{r}}\left(\frac{b \eta_{\mathrm{m}}}{\eta_{\mathrm{r}}+b \eta_{\mathrm{m}}}\right)^{2}
\end{aligned}
$$


and

$$
\begin{aligned}
\zeta_{\mathrm{m}} & =\frac{\partial \rho_{\mathrm{m}} / \partial \ln T}{\Gamma_{\mathrm{m}}}\left(\frac{T}{m}-\frac{\delta p}{\delta \rho}\right)^{2} \\
& =\frac{4}{3} b \eta_{\mathrm{m}}\left(\frac{\eta_{\mathrm{r}}}{\eta_{\mathrm{r}}+b \eta_{\mathrm{m}}}\right)^{2}
\end{aligned}
$$

since

$\delta \rho=12 \eta_{\mathrm{r}}+\frac{m^{2}}{T^{2}} \eta_{\mathrm{m}}$

$\delta p=4 \eta_{\mathrm{r}}+\frac{m}{T} \eta_{\mathrm{m}}$

This yields for the contribution (13) to (12):

$\zeta \sim \frac{4}{3} \frac{\eta_{\mathrm{r}} b \eta_{\mathrm{m}}}{\eta_{\mathrm{r}}+b \eta_{\mathrm{m}}}$.

It is readily seen, for the contributions (13) to $\zeta$, that $\zeta_{\mathrm{r}} \leq \frac{4}{3} \eta_{\mathrm{r}}$. One has also $\zeta_{\mathrm{m}} \leq \frac{4}{3} b \eta_{\mathrm{m}}$, but this is not a useful relation since $b \gg 1$. A better bound can be obtained by noting that, whatever the value of $b \eta_{\mathrm{m}}$, one has $\zeta_{\mathrm{m}} \leq \frac{1}{3} \eta_{\mathrm{r}}$, so the damping constraints obtained from $\zeta_{\mathrm{m}}$ do not lead to any new constraint once the damping due to $\eta_{\mathrm{r}}$ is taken into account. This is natural since Eq. (22) implies $\zeta<\frac{4}{3} \eta$ : so, when the constraints obtained from $\eta$ are satisfies, there are no new ones which appear due to $\zeta$.

The bound $\zeta<\frac{4}{3} \eta$ still holds when the contribution (19) is added to $\zeta$ : the bulk viscosity is not expected to bring in any stronger constraint than the shear viscosity.

\subsection{Decoupling and interaction rates}

In Sect. 2, we gave the transport coefficients as a sum over all species $i$ to which Dark Matter is coupled. The coupling condition may be written as $\Gamma_{\mathrm{dm}-i}>H$. More generally, a species $j$ is coupled to a species $i$ when $\Gamma_{j i}>H$. This condition ensures that the fluctuations of the particles $j$ follow those of particles $i$. More specifically this ensures that the fluctuations of particles $j$ will be erased at the same scale as those of species $i$.

The interaction rates $\Gamma_{\mathrm{dm}-i}$ and $\Gamma_{i-\mathrm{dm}}$ are in principle not equal. There are several reasons for this. First, the number of Dark Matter particles and of particles belonging to species $i$ may be different. Also, the relevant cross-section $\langle\sigma v\rangle_{\mathrm{dm}-i}$ is weighted by the momentum which is exchanged during the collision. This transfer may be quite different, depending on whether one considers the momentum transfer to a Dark Matter particle hitting a particle of species $i$ or whether it is the converse.

From the conservation of momentum, the relation between those two interaction rates can be written as

$\phi_{i} \Gamma_{i j}=\phi_{j} \Gamma_{j i}$

with $\phi_{i}=\rho_{i}+p_{i}$. So, the interaction rates $\Gamma_{i j}$ and $\Gamma_{j i}$ are quite naturally seen to be different. The condition $\Gamma_{\mathrm{dm}-i}>H$, for instance, does not necessarily imply $\Gamma_{i-\mathrm{dm}}>H$. This leaves also the possibility for $\Gamma_{i-\mathrm{dm}}<H$ and $\Gamma_{\mathrm{dm}-i}>H$, or more specifically $\Gamma_{i-\mathrm{dm}}<\Gamma_{i}<H<\Gamma_{\mathrm{dm}-i}$. In this case, when the coupling between Dark Matter and species $i$ is efficient enough to modify the properties of the Dark Matter fluid but not that of species $i$, one expects the damping of the freely-propagating species $i$ to be transmitted to the Dark Matter fluctuations. This, of course, occurs only in some range of parameters.

The relation (23) is due to the internal weightings within the statistical averages $\langle\overline{\sigma v}\rangle$ defined by Eq. (4) since the interaction probability is weighted by the momentum transferred. This is to contrast with what we denote as the $\langle\sigma v\rangle$ cross-section which is the usual cross-section which counts the number of interactions. The averages $\langle\overline{\sigma v}\rangle_{i j}$ and $\langle\overline{\sigma v}\rangle_{j i}$ in general are not equal:

$\phi_{i} n_{j}\langle\overline{\sigma v}\rangle_{i j}=\phi_{j} n_{i}\langle\overline{\sigma v}\rangle_{j i}$

so that $E_{i}\langle\overline{\sigma v}\rangle_{i j} \sim E_{j}\langle\overline{\sigma v}\rangle_{j i}$ where $E_{i}$ is the average energy per particle. Whether species $i$ is relativistic or not, one can write $E \sim 3 T c^{2} / v^{2}$, so for species $i$ and $j$ at the same temperature, there is the rule $v_{j}^{2}\langle\overline{\sigma v}\rangle_{i j} \sim v_{i}^{2}\langle\overline{\sigma v}\rangle_{j i}$. In the rest frame of the universe (where the blackbody radiation is isotropic), light particles hitting a much heavier particle will undergo substantial scattering, so each collision is associated to a transfer of momentum, and $\langle\overline{\sigma v}\rangle$ is of the order of $\langle\sigma v\rangle$. On the other hand, heavy particles hitting the light ones are barely scattered and there is little momentum transfer, so $\langle\overline{\sigma v}\rangle$ is, as compared to $\langle\sigma v\rangle$, down by a factor $v^{2} / c^{2}$, much lower than unity.

The same phase-space arguments obviously hold for the interaction rates governing the transport coefficients. This is illustrated by a well-known result for non relativistic particles (see e.g. Balian 1992). The rate $\left.\langle\overline{\sigma v}\rangle_{i j} \sim\langle\sigma v\rangle\right|_{i j} \equiv\left\langle\left.\sigma v\right|_{j i}\right.$ appears in the Lorentz approximation for light particles $i$ hitting heavy ones $j$. The rate to be used for brownian motion, when one considers heavy particles $j$ moving in the medium of the light ones $i$, is $\langle\overline{\sigma v}\rangle_{j i} \sim m_{i} /\left.m_{j}\langle\sigma v\rangle\right|_{i j}$. Indeed, since $m_{i} v_{i}^{2} \sim m_{j} v_{j}^{2}$ for species at the same temperature, the latter cross-section is down by $v_{j}^{2} / v_{i}^{2}$, as above.

\subsection{Time scales, linear response and the onset of transparency}

Transport theory, as outlined above, relies on several assumptions which are somewhat entangled.

First, the time-scale between the microscopic collisions (which for the present discussion we schematise by a unique rate $\Gamma$ ) that is $\tau=1 / \Gamma$, must be much shorter than the typical time $t=1 / H$ (where $H$ is the Hubble rate) during which the system evolves:

$\tau H \ll 1$.

The existence of a short time scale is a thermodynamical requirement based on two grounds: it insures local equilibrium which is the basis for the existence of transport coefficients, but also it allows the resolution of the Boltzmann equation to first order with respect to $\tau$ to calculate these coefficients.

Second, in order to avoid correction from General Relativity (the generalization to relativistic fluids in the previous sections incorporates only Special Relativity), both the mean-free-path $\sim v / \Gamma$ and the distance $l \sim v /(\Gamma H)^{1 / 2}$ traveled by a particle must be smaller than the horizon scale, $l_{\text {hor }} \sim c / H$. This condition is satisfied if Eq. (25) holds, but is required on totally different grounds. 
A third requirement is that the transport coefficients are small enough. Indeed, a modification of the global equilibrium generates a spatial gradient in the local thermodynamical variables. This in turn induces a transport of the corresponding thermodynamical quantity. The very definition of the transport coefficients stems from the assumption of a linear response to the deviation, i.e. that the associated flux is a linear function of the spatial gradient. The coefficient of this linear response being the relevant viscosity coefficient, the latter, measured ${ }^{5}$ in units of $\kappa \sim v^{2} / \Gamma$, must be small enough (by comparing this expression with the natural scale $c^{2} / H$, we also find the criterion (25)).

Clearly, for interaction rates small enough, one or the other - if not all - of the previous assumptions breaks down. However, this can be cured all at once by using the full Boltzmann equation, (Peebles \& Yu 1970; for recent references see e.g. Ma \& Bertschinger 1995; Hu \& White 1997; or Hu 1995), with the relevant collision terms. This approach provides the adequate continuation from the collisional regime to the regime of free propagation. The outcome of such numerical calculations is simple to predict: the transport, and thus the damping, which occurs over the distance traveled by the particles, should change gradually from $l \sim v /(\Gamma H)^{1 / 2}$ for $\Gamma \gg H$ to $l \sim v / H$ for $\Gamma \ll H$, with a transition around $\Gamma \sim H$. The actual investigation shows that the transport coefficients approach, based on the linear response theory and the general relativity (the latter being used to calculate the effect of the viscous terms but not to describe the corresponding internal physical processes which yield these terms), is rather accurate - within factors of order unity - up to $\Gamma \geq H$. The damping up to $\Gamma=H$ may be calculated along the lines outlined in Weinberg (1971), using the collisional result $l \sim v /(\Gamma H)^{1 / 2}$ (Peebles 1980) while for $\Gamma<H$ the damping is due (Davis et al. 1981) to the mixing over the distance the particles travel while freely propagating, $l \sim v / H$.

Rather than the truly accurate, but numerical, Boltzmann approach, we adopt the simplification described above to deal with the change in regimes. This will be sufficient for our purpose. Therefore, in this paper, we shall i) assume a sudden transition between the collisional and free streaming regimes; ii) use the collisional results up to the transition set at $\Gamma=$ $H$; and iii) assume a sudden onset of free streaming beyond the transition point. Expressions derived along this line will be given in Sect. 3 and applied to astrophysical processes in Sects. 4-6.

Another way to deal with the above restrictions when $\Gamma$ gets too small, i.e. when the transport coefficients get too large, is to consider that the response to a perturbation is still linear in the perturbation, but not in the transport coefficients. It may be achieved, requiring full relativistic invariance of the equations and preserving the Second Law of thermodynamics, i.e. the monotonic behaviour of the entropy (Israel \& Stewart 1979; a recent review may be found in Jou et al. 2001), by adding terms proportional to the time derivative (roughly $\propto H$ )

\footnotetext{
5 As an anticipation of the results obtained in Sect. 3, we note that the length $l$ also provides the measure of the scale $\propto(\kappa H)^{1 / 2}$ over which all inhomogeneities are smeared out, that is of their damping scale.
}

of the response multiplied by the collision time $\tau$. These terms obviously are negligible when the above conditions, schematised by Eq. (25), are satisfied. They turn out to be such as to provide the needed limitation when the interaction time and hence the transport coefficients become too large. This approach is called "Extended Thermodynamics" or also "Causal Thermodynamics". Applied to the present case, it corrects for the second and third above problems, but not for the first one $^{6}$. It is suited to the case where one remains clearly on the collisional side, with local equilibrium. Only the Boltzmann equation provides the full continuation across the decoupling regime into the free streaming domain. An excellent introduction to the matters is provided by Maartens (1996).

In the simple, but generic, example that is given in Maartens (1996), the analogue of our interaction rate $\Gamma$ turns out to be replaced by an effective quantity which in our case is $\Gamma^{*} \sim \Gamma(1+\tau H)$, arising from the terms which are nonlinear in the transport coefficient. This yields obviously an interpolation between the collisional and the free streaming estimates made above. The effective collision time is then given by $\tau^{*} \sim v /(\Gamma+H)$ while the analogue of our measure $\kappa$ (the magnitude of the transport coefficients) is changed into $\kappa^{*} \sim v^{2} /(\Gamma+H)$. In addition, the distance over which the particles travel, and hence the damping lengths, are then measured in units of $l^{*} \sim v /[(\Gamma+H) H]^{1 / 2}$. Despite the caveat given above, these estimates are seen to provide a nice continuation from the collisional towards the free-streaming regime when $\Gamma$ approaches $H$.

The difference with our sudden approximation is seen to be of order unity. Taking into account that we will have to deal with interaction rates and particle masses (velocities) which vary over tens of orders of magnitude (for any kind of Dark Matter), our approximation - albeit rather simple appears more than enough. The findings of the "Extended Thermodynamics" however provide a comforting confirmation for the relevance of the procedure that we have chosen to adopt.

\section{Damping scales associated with Dark Matter fluctuations}

The transport coefficients defined previously are associated with dissipative effects. The latter wash out all the Dark Matter primordial fluctuations having a size smaller than a wellspecified scale, that we aim to calculate in this section.

\footnotetext{
${ }^{6}$ One has also to redefine the concept of transport coefficients if one is willing to deal with the case where the collision time is not infinitesimally small as compared to the time evolution of the system, since the local equilibrium as well as the resolution of the Boltzmann equation to first order in the collision time to get these transport coefficients are then questioned. Note this can be in principle handled using a method outlined in Sect. 5.7 of Jou et al. (2001). The latter method amounts to consider the original move which led to the "Extended Thermodynamics" as the first order of systematic corrections and to repeat the procedure, up to infinite order. The latter procedure - which seems rather heavy - is specifically needed in order to restore (as is compulsory in the case of free propagation) the memory of the system which in this approximation scheme is lost at any finite order.
} 


\subsection{The collisional damping length}

Our basic (and sole assumption) is that there exists an epoch where the Dark Matter particles are in statistical equilibrium; this implies that $\Gamma_{\mathrm{dm}}=\sum_{i} \Gamma_{\mathrm{dm}-i}>H$ is, at some time, satisfied. Due to the evolution of the Universe, one expects $\Gamma_{\mathrm{dm}}$ to decrease and eventually to get smaller than the Hubble rate at a time $t_{\mathrm{dec}(\mathrm{dm})}$.

During all the period where the condition $\Gamma_{\mathrm{dm}}>H$ is satisfied, Dark Matter is collisional and all the associated primordial fluctuations with a size smaller than the collisional damping length are expected to be erased.

This length may be obtained explicitly in terms of the transport coefficients (Weinberg 1971). We write it as

$l_{\mathrm{cd}}^{2}=\pi^{2} \int^{t_{\mathrm{dec}(\mathrm{dm})}} \frac{\zeta+\frac{4}{3} \eta+\lambda T \frac{\rho_{\mathrm{m}}^{2}}{4 \phi \rho_{\mathrm{r}}}}{\not a^{2}} \mathrm{~d} t$.

Here $\phi=\sum_{i} \phi_{i}$. We have adopted the normalization of Efstathiou \& Silk (1983) so that the mass-scale associated with the length $l_{\mathrm{cd}}$ is given by $M_{\mathrm{cd}}=(4 \pi / 3) \rho_{\mathrm{m}} l_{\mathrm{cd}}^{3}$. The integral runs over all the period during which Dark Matter is collisional. For any reasonable energy-dependence of the cross-sections (see discussion in Paper II), the integral (26) is dominated by late times, so that the value of $l_{\mathrm{cd}}$ is determined by the Dark Matter decoupling.

Each transport coefficients $\zeta, \eta, \lambda T$ is given by a sum over $i$. Here $i$ denotes the species to which Dark Matter is coupled including Dark Matter itself. A given species drops out of the sum at a decoupling time $t_{\mathrm{dec}(\mathrm{dm}-i)}\left(\leq t_{\mathrm{dec}(\mathrm{dm})}\right)$. This time corresponds to the epoch at which Dark Matter ceases to be coupled to $i$. The damping scale then reads:

$l_{\mathrm{cd}}^{2}=\pi^{2} \sum_{i} \int^{t_{\mathrm{dec}(\mathrm{dm}-i)}} \frac{\zeta_{i}+\frac{4}{3} \eta_{i}+\lambda_{i} T \frac{\rho_{\mathrm{m}}^{2}}{4 \phi \rho_{\mathrm{r}}}}{\not a^{2}} \mathrm{~d} t$,

where $i$ runs over all the species to which Dark Matter is coupled (note that for $i=\mathrm{dm}$ one has to replace $t_{\mathrm{dec}(\mathrm{dm}-i)}$ by $\left.t_{\mathrm{dec}(\mathrm{dm})}\right)$.

As discussed in Sect. 2.3, we can omit the bulk viscosity coefficient which is either negligible or at most of the same efficiency than the shear viscosity coefficient. Therefore, it will not provide any new constraints.

Three different physical mechanisms can be at the origin of damping due to interactions, as outlined in Boehm et al. (2001):

1. the damping due to the interaction and displacement of the Dark Matter particles within the fluid. This is the selfdamping;

2. the damping due to a coupling between Dark Matter and a collisional species $i$ which experiences its own collisional damping. The Dark Matter then, within conditions we will discuss, acquires the damping of this species. This is the induced-damping;

3. the damping due to a coupling between Dark Matter and a freely-propagating species $i$, as discussed in Sect. 2.4. The origin of the damping of species $i$ in this case is the usual free-streaming damping. This damping is in the present case expected to be transmitted to Dark Matter. We will call such a damping the mixed-damping. It can be interpreted as a part (or a particular case) of the induced-damping.

\subsection{Self-damping and induced-damping}

To get the corresponding damping length, it is convenient to separate the sum over all species $i$ into a sum over the Dark Matter contribution dm and over $i \neq \mathrm{dm}$, that is over all species except Dark Matter. Equation (26) can then be split into two parts. One which only depends on the Dark Matter properties (corresponding to the self-damping length) while the other one depends on the characteristics of species $i \neq d m$ (corresponding to the induced-damping length). This yields

$l_{\mathrm{cd}}^{2}=l_{\mathrm{sd}}^{2}+\sum_{i \neq \mathrm{dm}} l_{\mathrm{id}}^{2}$

noting that the damping contributions combine quadratically. Using the explicit expressions of $\eta_{i}$ and $\lambda_{i} T$ given by Eqs. (6) and (8), one finds

$l_{\mathrm{sd}}^{2}=\frac{2 \pi^{2}}{3} \int_{0}^{t_{\mathrm{dec}(\mathrm{dm})}} \frac{\rho_{\mathrm{dm}} v_{\mathrm{dm}}^{2} t}{\not \phi a^{2} \Gamma_{\mathrm{dm}}}\left(1+\Theta_{\mathrm{dm}}\right) \frac{\mathrm{d} t}{t}$,

and for each species $i \neq \mathrm{dm}$ :

$l_{i \mathrm{~d}}^{2}=\frac{2 \pi^{2}}{3} \int_{0}^{t_{\mathrm{dec}(\mathrm{dm}-i)}} \frac{\rho_{i} v_{i}^{2} t}{\not a^{2} \Gamma_{i}}\left(1+\Theta_{i}\right) \frac{\mathrm{d} t}{t}$.

The factor

$\Theta_{i}=\frac{\rho_{\mathrm{m}}^{2}}{4 \phi \rho_{\mathrm{r}}} \frac{\partial \ln \rho_{i}}{\partial \ln T_{i}}$,

obtained by using Eqs. (8) and (26) is associated with the heat conduction coefficient. Here, it has been calculated neglecting the pressure of non-relativistic particles. As already seen in (Boehm et al. 2001), this yields $\lambda_{j} \propto \frac{\partial \ln \rho_{j}}{\partial \ln T_{j}}=\frac{\partial \ln m_{j} n_{j}}{\partial \ln T_{j}}=0$ for a non-relativistic species $j$. The heat conduction coefficient is then non-zero only for ${ }^{7}$ a relativistic species $i\left(\Theta_{i} \propto \frac{\rho_{\mathrm{m}}^{2}}{\phi \rho_{\mathrm{r}}}\right)$ and is important (i.e. $\Theta_{i}$ larger than unity) only in the matterdominated era where we have for any relativistic species $i$ the relation $\Theta_{i} \sim \frac{\rho_{\mathrm{m}}}{\rho_{\mathrm{r}}} \gg 1$ and $\frac{\rho_{i}}{\rho_{\mathrm{m}}} \Theta_{i} \sim \frac{\rho_{i}}{\rho_{r}} \leq 1$.

It turns out to be quite useful - and sufficient within the accuracy of our calculations - to approximate the integrals (29) and (30), written as $\int K \frac{\mathrm{d} t}{t}$ by the maximum the factor $K$ reaches within the integration interval:

$\int K \frac{\mathrm{d} t}{t} \simeq \operatorname{Max}[K]$

It is then straightforward to see that these integrals are dominated by the late times. Indeed, the decoupling corresponds to an epoch where the system passes from a coupled to an uncoupled regime while the Universe is expanding. This implies that $K$ is a growing function of time, as can be readily inferred from a short calculation counting the powers of $t$.

\footnotetext{
7 The complete expression including the pressure of the matter component can be derived along the lines of Weinberg (1971) but it is not needed in the present paper.
} 
The approximation (32), which aims to compare processes that differ by orders of magnitude, is good within factors of order unity. It considerably simplifies, at a very low cost, the overall classification of all kinds of Dark Matter we undertake in this paper.

As a result, the self-damping scale may be written as

$\left.l_{\mathrm{sd}}^{2} \sim \frac{2 \pi^{2}}{3} \frac{\rho_{\mathrm{dm}} v_{\mathrm{dm}}^{2} t}{\not \phi a^{2} \Gamma_{\mathrm{dm}}}\left(1+\Theta_{\mathrm{dm}}\right)\right|_{\mathrm{dec}(\mathrm{dm})}$,

while the induced-damping due to species $i$ reads

$\left.l_{i \mathrm{~d}}^{2} \sim \frac{2 \pi^{2}}{3} \frac{\rho_{i} v_{i}^{2} t}{\not a^{2} \Gamma_{i}}\left(1+\Theta_{i}\right)\right|_{\operatorname{dec}(\mathrm{dm}-i)}$.

It is convenient to rewrite these approximate expressions by introducing explicitly the Hubble rate (written as $H=\dot{a} / a=$ $\alpha / t$, with $\alpha=1 / 2$ or $2 / 3$ depending on whether the Universe is in its radiation or matter-dominated era). This yields

$\left.l_{\mathrm{sd}} \sim \pi r_{\mathrm{dm}}\left(\frac{H}{\Gamma_{\mathrm{dm}}}\right)^{\frac{1}{2}} \frac{v_{\mathrm{dm}} t}{a}\right|_{\mathrm{dec}(\mathrm{dm})}$,

and

$\left.l_{i \mathrm{~d}} \sim \pi r_{i}\left(\frac{H}{\Gamma_{i}}\right)^{\frac{1}{2}} \frac{v_{i} t}{a}\right|_{\operatorname{dec}(\mathrm{dm}-i)}$

where we have kept the factor $H / \Gamma$ (although it is unity at the Dark Matter decoupling) in order to extend it to the specific situation discussed in Sect. 4.2.3. Here, we have introduced the dimensionless ratios

$r_{\mathrm{dm}}=\left[\frac{2 \rho_{\mathrm{dm}}}{3 \alpha \phi}\left(1+\Theta_{\mathrm{dm}}\right)\right]^{\frac{1}{2}}$,

and

$r_{i}=\left[\frac{2 \rho_{i}}{3 \alpha \phi}\left(1+\Theta_{i}\right)\right]^{\frac{1}{2}}$.

From the relation $\rho_{i} \leq \not \rho$ and the discussion in Sect. 3.1 of the possible values that the factors $\Theta_{\mathrm{dm}}, \Theta_{i}$ may take, one can readily see that $r_{\mathrm{dm}}$ and $r_{i}$ are smaller or equal to unity.

Note that our formulas are valid for energy-dependent cross-sections, that is for temperature-dependent averages $\langle\overline{\sigma v}\rangle$.

\subsection{Free-streaming}

After its decoupling at time $t_{\operatorname{dec}(i)}$, species $i$ is freelypropagating. Its primordial fluctuations experience the socalled free-streaming damping. The damping acquired at a given time $t$ is proportional to the average distance traveled by a particle of the species, that is in comoving units:

$l_{\mathrm{fs}(i)} \propto \int_{t_{\mathrm{dec}(i)}}^{t} \frac{v_{i} \mathrm{~d} t}{a}$.

The free-streaming damping length is in some cases by convention taken as being exactly given by Eq. (39). It has also been argued, and checked numerically, that an interesting approximation to this damping length is given by (Bond \& Szalay 1983):

$l_{\mathrm{fs}(i)} \simeq \pi \operatorname{Max}\left[\frac{v_{i} t}{a}\right]$

where Max denotes the maximum value of the free-streaming scale within the integration interval $\left[t_{\operatorname{dec}(i)}, t\right]$ of Eq. (39). In the regime where we write our constraints, the two approximations are not dramatically different, and one might as well use the simpler one (40). With the normalization (40), the freestreaming length $l_{\mathrm{fs}}$ is associated (Bond \& Szalay 1983) with the damping of all the fluctuations of mass scale

$M=(4 \pi / 3) \rho_{\mathrm{m}} l_{\mathrm{fs}}^{3}$.

The quite simple approximation (40) is well-adapted for the comparison with the collisional damping length which is also approximately evaluated by means of a similar simplification (32).

As a rule, close to the parameter values (mass and interaction rate) adapted to our constraints, Eq. (39) yields length scales measured in the same dimensioned factors as the ones obtained from Eq. (40), but with numerical coefficients larger by a few units due to logarithmic contributions (but then it is not clear whether the mass-scale/length-scale relation is still Eq. (41) or the one where $l_{\mathrm{fs}}$ is to be divided by 2). For the sake of comparison, we give in Appendix D.1 the expression of the free-streaming lengths calculated by means of the approximation (39). The latter might be more suited than Eq. (40) far from the parameter values we handle in this work.

\subsection{Mixed-damping}

A freely-propagating species may transfer its own damping to the Dark Matter fluctuations if the condition $\Gamma_{\mathrm{dm}-i}>H>\Gamma_{i}>$ $\Gamma_{i-\mathrm{dm}}$ is satisfied. This in particular requires that $\phi_{i}>\phi_{\mathrm{dm}}$, due to the symmetries of $\Gamma_{i-j}$ under the permutation of $i$ and $j$, as can be inferred from Eq. (23). One can then decompose the induced-damping scale into two parts.

$$
\begin{aligned}
l_{i \mathrm{~d}}^{2}= & \frac{2 \pi^{2}}{3} \int^{t_{\mathrm{dec}(i)}} \frac{\rho_{i} v_{i}^{2}}{\not \rho a^{2} \Gamma_{i}}\left(1+\Theta_{i}\right) \mathrm{d} t \\
& +\frac{2 \pi^{2}}{3} \int_{t_{\mathrm{dec}(i)}}^{t_{\mathrm{dec}(\mathrm{dm}-i)}} \frac{\rho_{i} v_{i}^{2}}{\phi a^{2} H}\left(1+\Theta_{i}\right) \mathrm{d} t .
\end{aligned}
$$

The first term, valid as long as $\Gamma_{i}>H$, up to the time at which $i$ ceases to be collisional, is the (collisional) damping acquired by $i$ which is communicated to the Dark Matter. The second one is an estimate of the damping acquired by $i$ during the period where Dark Matter remains coupled to this species, now freelypropagating since $\Gamma_{i}<H$. It is obtained by simply replacing $\Gamma_{i}$ by $H$ to get an expression where the distance covered by a particle of species $i$ while it was collisional is replaced by the distance it covers when being free-streaming (this may be usefully linked to the discussion in Sect. 2.5). The mixed-damping length may finally be written as:

$l_{\mathrm{md}}^{2}=\frac{2 \pi^{2}}{3} \int_{t_{\mathrm{dec}(i)}}^{t_{\mathrm{dec}(\mathrm{dm}-i)}} \frac{\rho_{i} v_{i}^{2}}{\not a^{2} H}\left(1+\Theta_{i}\right) \mathrm{d} t$. 
With the same approximation as in Eqs. (36) or (40), we get:

$\left.l_{\mathrm{md}} \sim \pi r_{i} \frac{v_{i} t}{a}\right|_{\mathrm{dec}(\mathrm{dm}-i)}$.

Omitting the factor $r_{i}$, one easily recognizes the free-streaming scale of the species $i$ indicating that the damping acquired by $i$ is indeed transferred to the Dark Matter. In fact, because we are considering a composite fluid, it is not the free-streaming of species $i$ which is communicated to the Dark Matter fluctuations but the free-streaming weighted by the factor $r_{i}$. This length finally contributes quadratically to the total damping scale $l_{\text {cd }}$.

One may however consider that Eq. (44) is a too naive estimate. It is then worth to note that a lower bound to the damping length may be obtained by only considering the damping acquired by the Dark Matter fluctuations during the collisional regime of species $i$. This contribution corresponds to the first term in Eq. (42) and represents a lower bound to the true damping length $l_{i \mathrm{~d}}$ since

$l_{i \mathrm{~d}}^{2}>\frac{2 \pi^{2}}{3} \int^{t_{\mathrm{dec}(i)}} \frac{\rho_{i} v_{i}^{2}}{\not \phi a^{2} \Gamma_{i}}\left(1+\Theta_{i}\right) \mathrm{d} t$.

This integral may be written, with the same approximation as in Eq. (3.1):

$l_{\mathrm{id}}>\left.\pi r_{i} \frac{v_{i} t}{a}\right|_{\operatorname{dec}(i)}$.

Such a result solely relies on the standard physics involved in the collisional regime. It differs from the mixed-damping estimate, Eq. (44), just by the time at which the factors are evaluated. The collisional bound is estimated at $t_{\operatorname{dec}(i)}$ at which the species $i$ enters the free-streaming regime while the mixeddamping is estimated at $t_{\operatorname{dec}(\mathrm{dm}-i)}$ the true epoch at which the Dark Matter is no longer influenced by $i$. This collisional lower bound may be much smaller than the actual contribution, that we expect to be closer to the mixed-damping estimate. The latter will provide substantially stronger constraints. So, for our estimates we will use only this length, although it relies on less conventional physics.

\subsection{Dark Matter damping due to its own free-streaming}

After its total decoupling, Dark Matter freely propagates erasing all fluctuations having a size smaller than its own freestreaming length. From Eq. (40), this is at time $t>t_{\mathrm{dec}(\mathrm{dm})}$

$l_{\mathrm{fs}(\mathrm{dm})} \simeq \pi \operatorname{Max}\left[\frac{v_{\mathrm{dm}} t}{a}\right]$,

where the maximum is to be taken within the time interval $\left[t_{\mathrm{dec}(\mathrm{dm})}, t\right]$ where Dark Matter is freely-propagating.

\subsection{Comparison of the free-streaming scales with the self-damping and induced-damping scales}

There is a striking similarity between the expressions of the free-streaming scale (40) and the self-damping scale (35).
This is simple to understand. Since the collisional damping scale is based on diffusion processes, the associated damping length is proportional to the distance traveled by collisional particles during their random walk. In a non expanding Universe, this distance is usually much smaller than the distance traveled during the free-propagation. However in the present case, due to the expansion of the Universe, it gets increasingly large up to the decoupling time. The collisional damping length is hence dominated by the late times, where the random walk marginally corresponds to the free propagation.

The self-damping length (35) is however still somewhat smaller than the Dark Matter free-streaming length (40) since the transport of heat or momentum is done with an efficiency translated in terms of the factor $r_{\mathrm{dm}}$ (smaller or equal to unity). We therefore have the relation

$l_{\mathrm{sd}} \leq l_{\mathrm{fs}(\mathrm{dm})}$

The factor $r_{i}$ being also smaller or equal to unity, we infer that the induced collisional damping due to species $i$ satisfies

$l_{i \mathrm{~d}} \leq l_{\mathrm{fs}}(i)$

where $l_{\mathrm{fs}}(i)$ is the free-streaming length (40) of the species $i$. Therefore, the induced-damping scale of Dark Matter fluctuations is not bounded by the distance traveled by the Dark Matter particles but rather by the distance covered by the most rapid particles to which Dark Matter is coupled. This is very important for massive Dark Matter particles in statistical equilibrium with relativistic species since, in this case, the Dark Matter induced-damping scale is bounded by $c t$ and not by $v_{\mathrm{dm}} t$. In this case, although the thermal velocity of such Dark Matter particles is relatively small, their coupling to relativistic species allows them to undergo substantial damping.

\section{Constraining the Dark Matter properties from free-streaming and self-damping}

The self-damping and free-streaming lengths happen to depend only on two physical Dark Matter parameters: the Dark Matter particles' mass $m_{\mathrm{dm}}$ and the Dark Matter interaction rate with the medium $\Gamma_{\mathrm{dm}}$. The dependence of the free-streaming damping length on the Dark Matter particles' mass is well-known, its dependence on the interaction rate is less obvious and is in some sense the new feature examined in this section.

The constraints we get in this section are obtained (there is one exception discussed later) at the time $t_{\mathrm{dec}(\mathrm{dm})}$ where Dark Matter decouples to any species $i$, including itself. The rate $\Gamma_{\mathrm{dm}}$ depends among others on the number density of the particles in the medium. Due to the expansion, this density, and whence the rate, decreases with increasing decoupling time, that is with increasing interaction strength. It turns out to be convenient to work with an interaction rate which, taken at this epoch of reference, is an increasing function of the corresponding crosssection. Therefore we will use (see also Sect. A.2.6) the "reduced" interaction rate $\widetilde{\Gamma}_{\mathrm{dm}}=\Gamma_{\mathrm{dm}} a^{3}$, where the most obvious time-dependence due to the number density evolution is removed. 


\subsection{Classification of the Dark Matter self-damping processes}

We subdivide the plane $\left(m_{\mathrm{dm}}, \widetilde{\Gamma}_{\mathrm{dm}}\right)$ into regions within which the free-streaming and self-damping lengths have different expressions. The relevant time-scales which determine the damping lengths of the Dark Matter primordial fluctuations are

- the Dark Matter decoupling time $t_{\mathrm{dec}(\mathrm{dm})}$ (at which Dark Matter has decoupled from all species);

- the epoch at which Dark Matter becomes nonrelativistic $t_{\mathrm{nr}}$;

- the usual epoch of equality $t_{\mathrm{eq}(\gamma+v)}$.

These times may be translated in terms of scale-factors $a_{\mathrm{dec}(\mathrm{dm})}$, $a_{\mathrm{nr}}$ and $a_{\mathrm{eq}(\gamma+v)}=\rho_{\gamma+v}\left(T_{0}\right) / \rho_{\mathrm{m}}\left(T_{0}\right)$. Their relative ordering defines six regions in the Dark Matter [mass/interaction rate] parameter space:

\begin{tabular}{|ll||}
\hline Region I & $a_{\mathrm{dec}(\mathrm{dm})}<a_{\mathrm{nr}}<a_{\mathrm{eq}(\gamma+v)}$ \\
Region II & $a_{\mathrm{nr}}<a_{\mathrm{dec}(\mathrm{dm})}<a_{\mathrm{eq}(\gamma+v)}$ \\
Region III & $a_{\mathrm{nr}}<a_{\mathrm{eq}(\gamma+v)}<a_{\mathrm{dec}(\mathrm{dm})}$ \\
& \\
\hline & \\
Region IV & $a_{\mathrm{dec}(\mathrm{dm})}<a_{\mathrm{eq}(\gamma+v)}<a_{\mathrm{nr}}$ \\
Region V & $a_{\mathrm{eq}(\gamma+v)}<a_{\mathrm{dec}(\mathrm{dm})}<a_{\mathrm{nr}}$ \\
Region VI & $a_{\mathrm{eq}(\gamma+v)}<a_{\mathrm{nr}}<a_{\mathrm{dec}(\mathrm{dm})}$ \\
\hline \hline
\end{tabular}

This classification provides a natural way to separate heavy from light, late from early decoupling Dark Matter particles. This also allows one to distinguish particles which are able to annihilate and those which are not. Region I includes in particular the standard Hot Dark Matter (HDM) scenario (like for instance neutrino Dark Matter). Region II, includes, among others, annihilating particles (e.g. Weakly Interacting Massive Particles). Region III is devoted to strongly interacting particles. The last three regions, for which $a_{\mathrm{eq}(\gamma+\gamma)}<a_{\mathrm{nr}}$, correspond to the less likely very light Dark Matter particles, with masses below a few eV.

These Regions are shown in Fig. 1. Explicit equations for their borderlines are given in Appendix C.1. We now give the expressions of the damping lengths in these regions, and the associated constraints, whose values also are displayed in Fig. 1. Analytical expressions of the damping lengths may be found in Appendix D.1.

\subsection{Self-damping and free-streaming lengths}

\subsubsection{Region I: $a_{\mathrm{dec}(\mathrm{dm})}<a_{\mathrm{nr}}<a_{\mathrm{eq}}(\gamma+v)$}

Since $a_{\mathrm{dec}(\mathrm{dm})}$ corresponds to the total decoupling of Dark Matter, annihilation processes by definition are frozen afterwards. The Dark Matter comoving number density then remains constant: $n_{\mathrm{dm}}\left(T_{\mathrm{nr}}\right) a_{\mathrm{nr}}^{3}=n_{\mathrm{dm}}\left(T_{0}\right)$. The former can then be translated in terms of energy density $\rho_{\mathrm{dm}}=m_{\mathrm{dm}} n_{\mathrm{dm}}$, which, at the non-relativistic transition $\left(a=a_{\mathrm{nr}}\right)$, is required

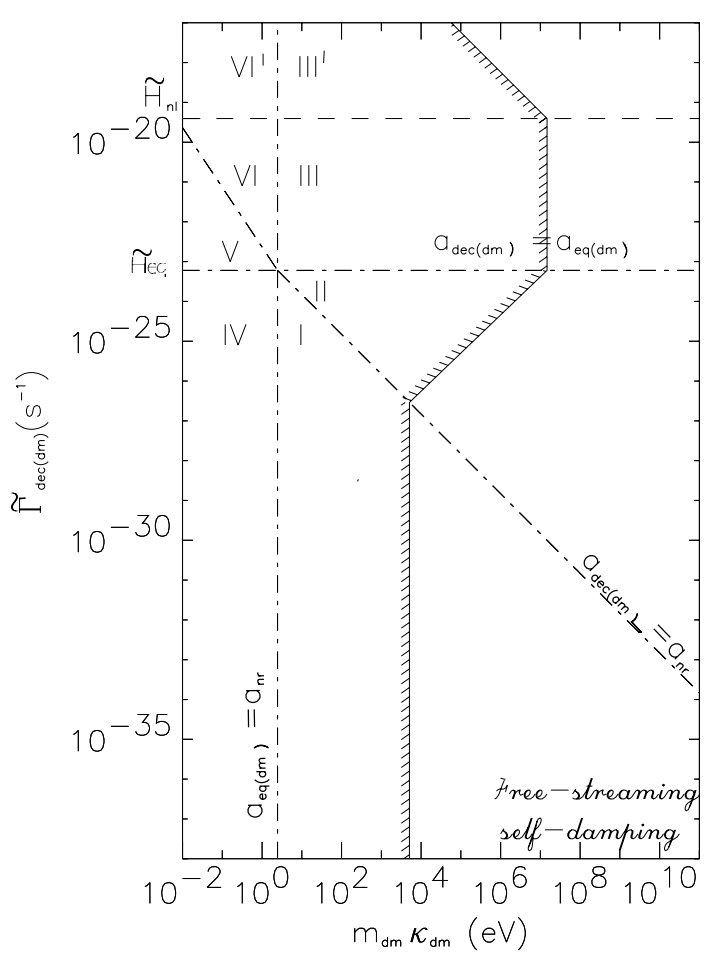

Fig. 1. Bounds in the (Dark Matter particles' mass, Dark Matter interaction rate) parameter space obtained from self-damping and free-streaming. The interaction rate $\widetilde{\Gamma}_{\mathrm{dec}(\mathrm{dm})}$ is $\Gamma_{\mathrm{dec}(\mathrm{dm})} a^{3}$ taken at the Dark Matter decoupling. The labels I to VI correspond to different scenarios. The hatches indicate which part of the parameter space is forbidden. It corresponds to the regions where the free-streaming and self-damping scales are above $100 \mathrm{kpc}$ (i.e. $\sim 10^{8} M_{\odot}$ ) scale. This is seen to provide a constraint involving both the interaction rate and the mass. The indications in this figure are schematic. Some factors in general of order unity are omitted for simplicity. The reader interested by these constraints should use the expressions in the text, where all factors are given explicitly.

to be equal to the energy-density ${ }^{8}$ in the relativistic regime: $\rho_{\mathrm{dm}}=\frac{1}{2} \epsilon g_{\mathrm{dm}} a_{B} T_{\mathrm{dm}}^{4} \equiv \frac{1}{2} g_{\star \mathrm{dm}} a_{B} T_{\gamma}^{4}$. This turns out to be a very stringent requirement. To be satisfied, it requires a quite small effective $g_{* \mathrm{dm}}$ to ensure that the number density of Dark Matter particles at the present epoch matches the observed relic abundance. Specifically

$g_{* \mathrm{dm}}=\frac{2 m_{\mathrm{dm}} n_{\mathrm{dm}}\left(T_{\mathrm{nr}}\right)}{\rho_{\gamma}\left(T_{\mathrm{nr}}\right)}=3.36 \frac{a_{\mathrm{nr}}}{a_{\mathrm{eq}(\gamma+v)}}$.

This condition may be rewritten in terms of $\kappa_{\mathrm{dm}}$, the factor which determines the ratio of the photon to Dark Matter temperature, as $\kappa_{\mathrm{dm}}^{3}\left(T_{\mathrm{nr}}\right)=\epsilon \frac{g_{\mathrm{dm}}}{3.36} \frac{a_{\mathrm{eq}(\gamma+\nu)}}{a_{\mathrm{nr}} \kappa_{\mathrm{dm}}\left(T_{\mathrm{nr}}\right)}$, that is

$\kappa_{\mathrm{dm}}\left(T_{\mathrm{nr}}\right) \sim 62\left(\epsilon \frac{g_{\mathrm{dm}}}{2}\right)^{\frac{1}{3}}\left(\frac{\Omega_{\mathrm{m}} h_{70}{ }^{2}}{0.3}\right)^{-\frac{1}{3}}\left(\frac{m_{\mathrm{dm}}}{1 \mathrm{MeV}}\right)^{\frac{1}{3}}$.

In this scenario, the Dark Matter number density is to be set at a quite remote epoch, and all changes in the particle number are frozen out, much before the non-relativistic transition. We

${ }^{8}$ The factors entering the energy-densities are defined precisely in Appendix A. 
Table 1.

$$
\begin{aligned}
& \overline{l_{\mathrm{sd}}^{(I)}=0.35 \mathrm{kpc} r_{\mathrm{dm}}\left(T_{\mathrm{dec}(\mathrm{dm})}\right) g^{\prime-1}\left(T_{\mathrm{dec}(\mathrm{dm})}\right) \frac{\widetilde{\Gamma}_{\mathrm{dec}(\mathrm{dm})}}{10^{-29} \mathrm{~s}^{-1}}}
\end{aligned}
$$

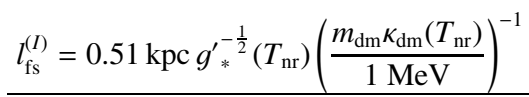

will thus call this way of matching the present relic density the Ultra-Relativistic Freeze-Out (URFO) scenario.

The requirement (51) may be achieved through various ways. One one hand, $\kappa_{\mathrm{dm}}$ may be large (quite larger than unity) if the decoupling between the Dark Matter (which may remain interacting) and the thermal bath occurs at a remote enough time, generically much before its total decoupling. On the other hand, one can have a small $\epsilon$ if the chemical reactions among Dark Matter particles which do not conserve the Dark Matter number density freeze out at early enough an epoch, the contact with the thermal bath being maintained. The particle number conservation from this epoch till nowadays then ensures that the Dark Matter density has the appropriate value at the non-relativistic transition and at the present epoch.

The expressions of the self-damping and free-streaming scales in this region read respectively:

$l_{\mathrm{sd}}^{(I)}=\left.\pi r_{\mathrm{dm}} \frac{c t}{a}\right|_{\mathrm{dec}(\mathrm{dm})}$

and

$l_{\mathrm{fs}}^{(I)}=\left.\pi \frac{c t}{a}\right|_{\mathrm{nr}}$.

Numerically, one finds the results given in Table 1 where $\widetilde{\Gamma}_{\mathrm{dec}(\mathrm{dm})}$ is the reduced Dark Matter interaction rate at $t_{\mathrm{dec}(\mathrm{dm})}$. The free-streaming scale corresponds to the result of Bond et al. (1980); Davis et al. (1981).

The comparison of Eq. (52) with Eq. (53) shows that the self-damping contribution is down by a factor

$\frac{\left.\frac{c t}{a}\right|_{\mathrm{dec}(\mathrm{dm})}}{\left.\frac{c t}{a}\right|_{\mathrm{nr}}}=\frac{a_{\mathrm{dec}(\mathrm{dm})}}{a_{\mathrm{nr}}}<1$.

The self-damping is therefore generically smaller than the freestreaming in Region I and will not be the most constraining effect. The coefficient $r_{\mathrm{dm}} \sim\left[\rho_{\mathrm{dm}}\left(T_{\mathrm{dec}(\mathrm{dm})}\right) / \phi\left(T_{\mathrm{dec}(\mathrm{dm})}\right)\right]^{1 / 2}$, is also below unity as long as Dark Matter is coupled to radiation. Indeed (as may be inferred more readily using the ratio of the energy-densities given in Sect. B.2) $\rho_{\mathrm{dm}} / \phi \sim \rho_{\mathrm{dm}} / \rho_{\mathrm{r}} \leq a_{\mathrm{nr}} / a_{\mathrm{eq}}$ implies $r_{\mathrm{dm}} \leq 1$. On the other hand, if the decoupling from radiation occurs before the Dark Matter total decoupling, the Dark Matter fluid does not contain any radiation component and one gets: $\phi \sim \rho_{\mathrm{m}}$ and $r_{\mathrm{dm}} \sim 1$.

From Eq. (53), we get the usual condition

$m_{\mathrm{dm}} \kappa_{\mathrm{dm}}\left(T_{\mathrm{nr}}\right)>5.1 \mathrm{keVg}_{*}^{\prime-\frac{1}{2}}\left(T_{\mathrm{nr}}\right)\left(\frac{l_{\mathrm{struct}}}{100 \mathrm{kpc}}\right)^{-1}$.

Implementing the estimated value (51) of $\kappa_{\mathrm{dm}}$, yields the condition

$m_{\mathrm{dm}}>0.9 \mathrm{keV}\left(\epsilon \frac{g_{\mathrm{dm}}}{2}\right)^{-\frac{1}{4}} g_{*}^{\prime-\frac{3}{8}}\left(\frac{\Omega_{\mathrm{m}} h_{70}{ }^{2}}{0.3}\right)^{\frac{1}{4}}\left(\frac{l_{\text {struct }}}{100 \mathrm{kpc}}\right)^{-\frac{3}{4}}$, at the origin of the so-called " $1 \mathrm{keV}$ " mass limit (Davis et al. 1981).

\subsubsection{Region II: $a_{\mathrm{nr}}<a_{\mathrm{dec}(\mathrm{dm})}<a_{\mathrm{eq}}(\gamma+v)$}

The Dark Matter (total) decoupling in Region II occurs after the non-relativistic transition. There are in this case two ways of satisfying the relic density requirement:

- The first one, as in Region I, is to consider the URFO scenario where the constraint (51) has to be imposed.

- The second one is to consider particles which remain in chemical equilibrium up to their non-relativistic transition (actually slightly after, as we shall see below). The allowance for this second possibility opens a new window. Indeed, one may have a large energy density before the nonrelativistic transition. Then the Dark Matter particles, still in chemical equilibrium, annihilate and the number density exponentially decreases. Once it reaches the appropriate value, one assumes that the reactions freeze-out. We will call this way of matching the present relic density the NonRelativistic Freeze-Out (NRFO) scenario.

In the NRFO scenario, to match the observed relic density, a specific value of the freeze-out temperature, and in turn of the annihilation cross-section (see e.g. Lee \& Weinberg 1977; Griest 1988) is required. We only shortly discuss its derivation, which is well-known. The conservation of the Dark Matter number density in the NRFO scenario is relevant only after the chemical decoupling. By equating the comoving Dark Matter number density during its exponential decrease (just before freeze-out) to the comoving density just after freeze-out, with

$T_{\mathrm{dm}}^{\mathrm{fo}}=\frac{m_{\mathrm{dm}}}{x_{\mathrm{fo}}}$,

one sees that $x_{\mathrm{fo}}$ obeys the equation

$x_{\mathrm{fo}}^{\frac{3}{2}} \mathrm{e}^{-x_{\mathrm{fo}}}=(2 \pi)^{\frac{3}{2}}\left(\frac{\hbar c}{T_{0}}\right)^{3} \frac{\widetilde{n}_{\mathrm{dm}}}{g_{* \mathrm{dm}} / \kappa_{\mathrm{dm}}^{3}\left(T_{\mathrm{fo}}\right)}$.

Yielding the very simple rule

$x_{\mathrm{fo}} \sim 14+\ln \frac{m_{\mathrm{dm}} \kappa_{\mathrm{dm}}\left(T_{\mathrm{fo}}\right)}{1 \mathrm{MeV}}$,

where we have neglected unimportant logarithmic factors. For $\sim 100 \mathrm{GeV}$ particles, $x_{\mathrm{fo}}$ is of the order of $\sim 20$ : the annihilation reactions freeze out somewhat after the non-relativistic transition, at $T_{\mathrm{dm}}^{\mathrm{fo}}=\frac{m_{\mathrm{dm}}}{20}$. This implies a well-defined value for the cross-sections to maintain the chemical equilibrium up to $x=x_{\mathrm{fo}}$ and not after. It yields the standard constraint on the Dark Matter parameters, which in this case must be satisfied instead of Eq. (51). Larger values of $\langle\sigma v\rangle_{\mathrm{ann}}$ are prohibited ${ }^{9}$, as well as lower values, unless they are small enough so as to comply with the URFO scenario and therefore satisfy condition (51). In the special case where the chemical equilibrium is

\footnotetext{
9 The values we mention here slightly differ in case of coannihilation.
} 
reached via direct annihilation, for instance, the requirement is found to be

$$
\begin{aligned}
\langle\sigma v\rangle_{\mathrm{ann}} & =\frac{H_{\mathrm{r}} x_{\mathrm{fo}} T_{0}}{m_{\mathrm{dm}} \kappa_{\mathrm{dm}}\left(T_{\mathrm{fo}}\right) \tilde{n}_{\mathrm{dm}}} \\
& \sim 7.6 \times 10^{-26} \mathrm{~cm}^{3} \mathrm{~s}^{-1} \frac{g^{\prime \frac{1}{2}}\left(T_{\mathrm{fo}}\right)}{\frac{\Omega_{\mathrm{dm}} h_{70}^{2}}{0.25} \kappa_{\mathrm{dm}}\left(T_{\mathrm{fo}}\right)} \frac{x_{\mathrm{fo}}}{20} .
\end{aligned}
$$

This value is nearly a universal constant owing our good knowledge of the density parameter $\Omega_{\mathrm{dm}}$ and the Hubble constant $H_{0}$. In particular it is almost independent of the Dark Matter particles' mass. Any Dark Matter particle that is able to annihilate must satisfy this condition in order not to overclose the Universe. This is true, in particular, for any particle having a mass above the MeV range.

The condition (60) however may be alleviated, to allow larger (but not smaller) values of $\langle\sigma v\rangle_{\mathrm{ann}}$. This is the case provided the particles and anti particles (with respective densities $n_{\mathrm{dm}+}$ and $\left.n_{\mathrm{dm}-}\right)$ exhibits an asymmetry before the nonrelativistic transition:

$\beta=\frac{n_{\mathrm{dm}+}-n_{\mathrm{dm}-}}{n_{\mathrm{dm}+}+n_{\mathrm{dm}-}}$

Indeed, the density at the relativistic side then is $\left.n_{\mathrm{dm}}\right|_{\mathrm{r}}=n_{\mathrm{dm}+}+$ $n_{\mathrm{dm}-}$ and particle number conservation then shows the number density at the non-relativistic side to be $\left.n_{\mathrm{dm}}\right|_{\mathrm{nr}}=n_{\mathrm{dm}+}-n_{\mathrm{dm}--}$. To produce the observed relic number density, it may be shown (see Appendix B.3) that a jump $\frac{\left.n_{\mathrm{dm}}\right|_{\mathrm{nr}}}{n_{\mathrm{dm} \mathrm{r}}}=\frac{a_{\mathrm{nr}}}{a_{\mathrm{eq}(\mathrm{dm})}}$ is required at the non-relativistic transition. The relic density condition (60) then is replaced by the condition

$\beta=\frac{a_{\mathrm{nr}}}{a_{\mathrm{eq}(\mathrm{dm})}}$.

We now estimate the damping scale associated to Dark Matter candidates belonging to region II. At total decoupling, Dark Matter particles have a velocity

$v_{\mathrm{dm}}\left(T_{\mathrm{dec}(\mathrm{dm})}\right)=f\left(\frac{a_{\mathrm{nr}}}{a_{\mathrm{dec}(\mathrm{dm})}}\right)^{\frac{1}{2}}$

where

$f=\left(\frac{\kappa_{\mathrm{dm}}\left(T_{\mathrm{nr}}\right)}{\kappa_{\mathrm{dm}}\left(T_{\mathrm{dec}(\mathrm{dm})}\right)}\right)^{\frac{1}{2}}$.

We then get

$l_{\mathrm{fs}}^{(\mathrm{II})}=l_{\mathrm{sd}}^{(\mathrm{II})} / r_{\mathrm{dm}}=\left.\pi \frac{v t}{a}\right|_{\mathrm{dec}(\mathrm{dm})}$.

The ratio $\rho_{\mathrm{dm}}\left(T_{\mathrm{dec}(\mathrm{dm})}\right) / \rho_{\mathrm{r}}\left(T_{\mathrm{dec}(\mathrm{dm})}\right) \sim a_{\mathrm{dec}(\mathrm{dm})} / a_{\mathrm{eq}}$ is still smaller than unity. But $r_{\mathrm{dm}}-$ which depends on the ratio $\rho_{\mathrm{dm}}\left(T_{\mathrm{dec}(\mathrm{dm})}\right) / \phi\left(T_{\mathrm{dec}(\mathrm{dm})}\right)$ of the species that are actually coupled- may be unity if the Dark Matter is not coupled to radiation, as already noted for Region I. The self-damping and free-streaming damping lengths in Region II finally take the values given in Table 2 .

The condition $l_{\mathrm{fs}}^{(I I)}<l_{\text {struct }}$ implies

$$
\begin{aligned}
m_{\mathrm{dm}} \kappa_{\mathrm{dm}}\left(T_{\mathrm{nr}}\right)> & 11 \mathrm{MeV} f^{2} g_{*}^{\prime-\frac{3}{2}}\left(T_{\mathrm{dec}(\mathrm{dm})}\right) \\
& \times \frac{\widetilde{\Gamma}_{\mathrm{dec}(\mathrm{dm})}}{6 \times 10^{-24} \mathrm{~s}^{-1}}\left(\frac{l_{\text {struct }}}{100 \mathrm{kpc}}\right)^{-2} .
\end{aligned}
$$

Table 2.

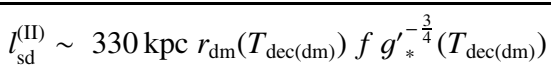

$$
\begin{aligned}
& \left(\frac{m_{\mathrm{dm}} \kappa_{\mathrm{dm}}\left(T_{\mathrm{nr}}\right)}{1 \mathrm{MeV}}\right)^{-\frac{1}{2}}\left(\frac{\widetilde{\Gamma}_{\mathrm{dec}(\mathrm{dm})}}{6 \times 10^{-24} \mathrm{~s}^{-1}}\right)^{\frac{1}{2}} \\
& l_{\mathrm{fs}}^{(\mathrm{III})} \sim 330 \mathrm{kpc} f{g^{\prime}}^{-\frac{3}{4}}\left(T_{\mathrm{dec}(\mathrm{dm})}\right) \\
& \left(\frac{m_{\mathrm{dm}} \kappa_{\mathrm{dm}}\left(T_{\mathrm{nr}}\right)}{1 \mathrm{MeV}}\right)^{-\frac{1}{2}}\left(\frac{\widetilde{\Gamma}_{\mathrm{dec}(\mathrm{dm})}}{6 \times 10^{-24} \mathrm{~s}^{-1}}\right)^{\frac{1}{2}}
\end{aligned}
$$

Table 3.

$$
\begin{aligned}
& l_{\mathrm{sd}}^{(I I I)} \sim 435 \mathrm{kpc}\left(\frac{\Omega_{\mathrm{m}} h_{70}{ }^{2}}{0.3}\right)^{-\frac{1}{2}} r_{\mathrm{dm}}\left(T_{\mathrm{dec}(\mathrm{dm})}\right) f\left(\frac{m_{\mathrm{dm}} \kappa_{\mathrm{dm}}\left(T_{\mathrm{nr}}\right)}{1 \mathrm{MeV}}\right)^{-\frac{1}{2}} \\
& \underline{l_{\mathrm{fs}}^{(I I I)} \sim 435 \mathrm{kpc}\left(\frac{\Omega_{\mathrm{m}} h_{70}{ }^{2}}{0.3}\right)^{-\frac{1}{2}} f\left(\frac{m_{\mathrm{dm}} \kappa_{\mathrm{dm}}\left(T_{\mathrm{nr}}\right)}{1 \mathrm{MeV}}\right)^{-\frac{1}{2}}}
\end{aligned}
$$

\subsubsection{Region III: $a_{\mathrm{nr}}<a_{\mathrm{eq}}<a_{\mathrm{dec}(\mathrm{dm})}$}

As in the previous case, we have to consider the URFO and NRFO scenarios. To get the observed Dark Matter energy density one has to impose the same relations (51), and (60) or (62), respectively, as for Region II.

The velocity of the Dark Matter particles at decoupling has the same expression as for region II. A straightforward calculation (which takes into account that the decoupling occurs while the universe is matter-dominated) provides the damping scales. The interesting point here is that, unlikely to region II, we expect the ratio $r_{\mathrm{dm}} \sim\left(\rho_{\mathrm{dm}} / \phi\right)^{\frac{1}{2}}$ to be of the order of unity (if not exactly equal to 1 ) since the density $\phi$ refers only to the species coupled to the Dark Matter and the decoupling occurs in the matter dominated era. Therefore self-damping and freestreaming are comparable in this region:

$l_{\mathrm{fs}}^{(\mathrm{III})}=l_{\mathrm{sd}}^{(\mathrm{III})} / r_{\mathrm{dm}}=\left.\pi \frac{v t}{a}\right|_{\operatorname{dec}(\mathrm{dm})}$,

which numerically yield the results given in Table 3 . Despite $l_{\mathrm{fs}}$ and $l_{\mathrm{sd}}$ are given by the same expression than in Region II, their evaluation yields a different result since after equality, in Region III, the time of decoupling grows less rapidly with the interaction rate. As a result, the damping lengths turn out to be independent of this rate.

This provides a range for the Dark Matter mass:

$m_{\mathrm{dm}} \kappa_{\mathrm{dm}}\left(T_{\mathrm{nr}}\right)>19 \mathrm{MeV}\left(\frac{\Omega_{\mathrm{m}} h_{70}{ }^{2}}{0.3}\right)^{-1} f^{2}\left(\frac{l_{\text {struct }}}{100 \mathrm{kpc}}\right)^{-2}$

A specific case: coupling up to the onset of structure formation. The previous expressions are only relevant provided decoupling occurs before structure formation, that is before the non-linear collapse of the primordial structures, assumed to take place at a scale-factor $a_{\mathrm{nl}}$. We leave the latter arbitrary, but it is expected to be roughly of the order of $\sim 1 / 10$ for objects with a size $\leq 10^{8} M_{\odot}$. 
For $a_{\mathrm{dec}(\mathrm{dm})}>a_{\mathrm{nl}}$, which may be rewritten as $\left.\widetilde{\Gamma}_{\mathrm{dm}}\right|_{a=a_{\mathrm{nl}}}>\widetilde{H}_{\mathrm{nl}}$ (the interaction rate $\widetilde{H}_{\mathrm{nl}}$ is given in Table A.2), there is no freestreaming at all before structure formation. Only collisional damping is at work up to the non-linear collapse, with

$l_{\mathrm{sd}} / r_{\mathrm{dm}}=\left.\pi\left(\widetilde{H}_{\mathrm{nl}} / \widetilde{\Gamma}_{\mathrm{dm}}\right)^{\frac{1}{2}} \frac{v t}{a}\right|_{\mathrm{nl}}$

that is

$$
\begin{aligned}
l_{\mathrm{sd}}^{(\mathrm{III})} \sim & 435 \mathrm{kpc}\left(\frac{\Omega_{\mathrm{m}} h_{70}{ }^{2}}{0.3}\right)^{-\frac{1}{2}} r_{\mathrm{dm}}\left(T_{\mathrm{dec}(\mathrm{dm})}\right) f \\
& \times\left(\frac{m_{\mathrm{dm}} \kappa_{\mathrm{dm}}\left(T_{\mathrm{nr}}\right)}{1 \mathrm{MeV}}\right)^{-\frac{1}{2}}\left(\frac{\widetilde{\Gamma}_{\mathrm{dm}}}{\widetilde{H}_{\mathrm{nl}}}\right)^{-\frac{1}{2}} .
\end{aligned}
$$

It is useful to note that $l_{\mathrm{sd}}^{\mathrm{III}}$ depends on the collision rate $\widetilde{\Gamma}_{\mathrm{dm}}\left(T_{\mathrm{nl}}\right)$ taken at the time of the onset of the non-linear gravitational collapse and no longer at decoupling. Also, $l_{\mathrm{sd}}^{\mathrm{III}}=$ $\left(\frac{\widetilde{H}_{\mathrm{nl}}}{\widetilde{\Gamma}_{\mathrm{dm}}}\right)^{\frac{1}{2}} l_{\mathrm{sd}}^{\mathrm{III}}$, Eq. (70), is smaller than $l_{\mathrm{sd}}^{\mathrm{III}}$, Eq. (3) although the corresponding Dark Matter interaction rate, taken at a given epoch, is larger.

For the self-damping to be acceptable, we must have

$$
\begin{aligned}
m_{\mathrm{dm}} \kappa_{\mathrm{dm}}\left(T_{\mathrm{nr}}\right)> & 19 \mathrm{MeV}\left(\frac{\Omega_{\mathrm{m}} h_{70}{ }^{2}}{0.3}\right)^{-1} r_{\mathrm{dm}}^{2}\left(T_{\mathrm{dec}(\mathrm{dm})}\right) f^{2} \\
& \times\left(\frac{\widetilde{\Gamma}_{\mathrm{dm}}}{\widetilde{H}_{\mathrm{nl}}}\right)^{-1}\left(\frac{l_{\text {struct }}}{100 \mathrm{kpc}}\right)^{-2} \cdot
\end{aligned}
$$

Pending a more complete discussion of this issue in Paper II, we may recall that the present requirements are necessary conditions, as are all the bonds we establish in this paper, irrespectively of other astrophysical conditions which may be needed to achieve an acceptable scenario with the assumed Dark Matter parameters.

\subsubsection{Region IV: $a_{\operatorname{dec}(\mathrm{dm})}<a_{\mathrm{eq}}<a_{\mathrm{nr}}$}

The Dark Matter particles are relativistic when they decouple. The analytic expression of the damping scales is the same as in Region I. The free-streaming length $l_{\mathrm{fs}}^{\mathrm{IV})}$ is still given by (53). The numerical value of the collisional damping scale however is different. Since we have here $\rho_{\mathrm{dm}} \sim \not$, the collisional damping length is still smaller than the free-streaming length, but by a factor $\left.\frac{c t}{a}\right|_{\mathrm{dec}(\mathrm{dm})} /\left.\frac{c t}{a}\right|_{\mathrm{nr}}=a_{\mathrm{dec}(\mathrm{dm})} / a_{\mathrm{nr}}$ only.

Requiring the free-streaming damping scale to be smaller than $l_{\text {struct }} \sim 100 \mathrm{kpc}$ still calls for Dark Matter particle masses in the keV range or above. This cannot be achieved in Region IV. Region IV is therefore excluded by the damping requirements.

\subsubsection{Region V: $a_{\text {eq }}<a_{\operatorname{dec}(\mathrm{dm})}<a_{\mathrm{nr}}$}

The calculation is strictly identical to the one done in the previous section for Region IV since $a_{\mathrm{dec}(\mathrm{dm})}=a_{\text {eq }}$, does no longer correspond to a change in the expansion regime. The transition from a radiation to a matter dominated universe at Dark Matter decoupling occurs indeed for $a_{\mathrm{dec}(\mathrm{dm})}=a_{\mathrm{nr}}$.

Region $\mathrm{V}$ is therefore also excluded by the damping requirements.

\subsubsection{Region VI: $a_{\mathrm{eq}}<a_{\mathrm{nr}}<a_{\mathrm{dec}}(\mathrm{dm})$}

Dark Matter decouples in the matter dominated era. The collisional, as well as the free-streaming scales are dominated by the contribution near the decoupling time. Since we have still $\rho_{\mathrm{dm}} \sim \phi$, their contribution is nearly the same as long as decoupling occurs before the epoch of non-linear gravitational collapse where the structures actually start to build up. The same calculation as in Region III is relevant in the present case. The conditions for the dm density to match the observed one still imposes (51): anticipating the astrophysical discussion of Paper II, we readily see that the URFO scenario is the only sensible case to consider. Indeed, in Region VI, the presentday number density of these light Dark Matter particles is much higher than the photon density. Such an unusually large number density can only be reached under very special circumstances. It does not make much sense to assume that it results from an even much larger one, which is followed by a period of annihilation. However, strictly speaking, this is not forbidden.

The damping scales are given by their expressions in Region III, with comparable effects from collisions and freestreaming. Masses above the $\mathrm{MeV}$ scale are therefore required to avoid prohibitive damping. But such masses are not allowed in Region VI (Fig. 1).

A specific case: coupling up to the onset of non-linear collapse. For $\widetilde{\Gamma}\left(T_{\mathrm{nl}}\right)>\widetilde{H}_{\mathrm{nl}}$, which we call Region VI', there is, on the other hand, never decoupling until the onset of the non-linear gravitational collapse. Only collisional damping is at work so the free-streaming constraint drops out and expression (70) of the self-damping length, as well as the constraint (71) hold in this case.

This leaves an allowed window in the parameter space of Region VI', for extremely large an interaction rate.

\section{Constraining the Dark Matter candidates from the neutrino induced-damping}

The expression of the induced-damping scale (36) shows that relativistic particles yield a large damping effect. They are expected to provide the most stringent constraints on the Dark Matter interaction rates. Since the obvious components of the radiation are photons and neutrinos, we specifically focus on these two species. These particles have the advantage that their dominant interaction (apart from the interaction with Dark Matter that we aim to discuss in the present paper) is with electrons. It is known and provides the scales of reference for the discussion of the damping effect induced by these particles.

In this section, we compute the collisional damping of Dark Matter fluctuations due to a possible coupling of Dark Matter with neutrinos and evaluate the corresponding constraints. The photon induced-damping of the Dark Matter fluctuations will be discussed in Sect. 6 .

\subsection{Dark Matter parameter space for neutrino induced damping}

The neutrino induced-damping scale depends on the total neutrino interaction rate with the medium $\Gamma_{v}=\sum_{j} \Gamma_{v-j}=\Gamma_{v-\mathrm{e}}+$ $\Gamma_{v-\mathrm{dm}}+\ldots$. In the standard scheme, this rate is dominated by the 
collisions with electrons, $\Gamma_{v} \sim \Gamma_{v-\mathrm{e}}$. The only possibility for $\Gamma_{v}$ not to be dominated by $\Gamma_{v-\mathrm{e}}$ is when $\Gamma_{v-\mathrm{dm}}>\Gamma_{v-\mathrm{e}}$.

Thanks to our approximations (36), or (44), the only other parameter which enters the calculation of the damping scale is the upper limit of the integral (42), namely the time $t_{\mathrm{dec}(\mathrm{dm}-v)}$ where Dark Matter decouples from neutrinos. This defines the reference time at which all our constraints on the interaction rate or on the cross-sections are obtained.

For the actual applications, it turns out to be convenient to note that both the interaction rate $\Gamma_{v-\mathrm{dm}}$ and time $t_{\mathrm{dec}(\mathrm{dm}-v)}$ may be expressed as a function of the "reduced" (see Sect. A.2.6) interaction rate $\widetilde{\Gamma}_{\mathrm{dm}-\gamma} \equiv \Gamma_{\mathrm{dm}-\nu} a^{3}$, or equivalently as a function of $\langle\overline{\sigma v}\rangle_{v-\mathrm{dm}} \equiv \widetilde{\Gamma}_{v-\mathrm{dm}} / \widetilde{n}_{\mathrm{dm}}$. The other parameter entering the calculation is the Dark Matter particles' mass $m_{\mathrm{dm}}$. This defines a two-parameter space which allows one to classify all kinds of Dark Matter particles which interact with neutrinos, say $\left[m_{\mathrm{dm}}, \widetilde{\Gamma}_{\mathrm{dm}-v}\right]$ or $\left[m_{\mathrm{dm}},\langle\overline{\sigma v}\rangle_{v-\mathrm{dm}}\right]$.

In the following, we shall distinguish "Regions" in this parameter-space which correspond to different analytical expressions of the neutrino induced-damping scale. All together, they cover the whole parameter space.

\subsubsection{Dark Matter decouples from neutrinos while the latter are collisional}

This is the case where

$\widetilde{\Gamma}_{\mathrm{dm}-v} \leq\left.\widetilde{\Gamma}_{v}\right|_{\mathrm{dec}(\mathrm{dm}-v)}$.

The Dark Matter then experiences a collisional damping induced by its coupling to neutrinos. Since the neutrino interaction rate can be dominated by the collision rate with either the electrons or with Dark Matter, we are led to distinguish two sub-cases A and B.

Region A. In this region, the neutrino interaction rate is dominated by interactions with electrons. This corresponds to:

$\widetilde{\Gamma}_{v-\mathrm{dm}} \leq\left.\widetilde{\Gamma}_{v-\mathrm{e}}\right|_{\operatorname{dec}(\mathrm{dm}-v)}$, that is $\widetilde{\Gamma}_{v} \sim \widetilde{\Gamma}_{v-\mathrm{e}}$.

In this case, the neutrino collisional damping is due to their interaction with electrons.

Region B. Here the neutrino interaction rate is dominated by interactions with Dark Matter. This corresponds to:

$\widetilde{\Gamma}_{v-\mathrm{dm}}>\left.\widetilde{\Gamma}_{v-\mathrm{e}}\right|_{\operatorname{dec}(\mathrm{dm}-v)}$, that is $\widetilde{\Gamma}_{v} \sim \widetilde{\Gamma}_{v-\mathrm{dm}}$.

The collisional damping is then due to the neutrino interactions with Dark Matter.

\subsubsection{Dark Matter decouples from neutrinos while the latter are freely-propagating}

In this situation, there is only one region, namely:

$$
\begin{aligned}
& \text { Region C } \\
& \widetilde{\Gamma}_{\mathrm{dm}-v}>\left.\widetilde{\Gamma}_{v}\right|_{\operatorname{dec}(v)} .
\end{aligned}
$$

Neutrinos are already free-streaming when Dark Matter decouples from the latter. This is the mixed-damping regime. The corresponding damping length has been estimated Eq. (44). Since the neutrino collision rate does not enter the mixeddamping expression there is no point in the present case to separate the case where $\widetilde{\Gamma}_{v-\mathrm{dm}}$ is smaller or larger than $\widetilde{\Gamma}_{v-\mathrm{e}}$.

\subsubsection{Borderlines}

The borderlines between regions $\mathrm{A}, \mathrm{B}$ and $\mathrm{C}$ are shown in Figs. 2-5, depending on whether one considers the URFO or the NRFO scenario, and whether one displays the parameterspace in the $\left[m_{\mathrm{dm}}, \widetilde{\Gamma}_{\mathrm{dm}-v}\right]$ or $\left[m_{\mathrm{dm}},\langle\overline{\sigma v}\rangle_{v-\mathrm{dm}}\right]$ coordinates. These borderlines are calculated explicitly in Appendix C.2. They are important since they define the conditions of validity of our expressions of the damping length.

\subsection{Neutrino induced-damping scales and limits on the interaction rates}

In the absence of significant Dark Matter interactions, as it is commonly assumed, neutrinos are expected to decouple from the thermal bath in the radiation dominated era. The last interactions are with electrons. If we adopt the standard neutrinoelectron interaction (see Sect. A.3.1), the neutrinos decouple at a temperature slightly above $1 \mathrm{MeV}$, when their interaction rate is of the order of $\widetilde{\Gamma}_{\operatorname{dec}(v-\mathrm{e})} \sim 2.8 \times 10^{-30} \mathrm{~s}^{-1}$. At neutrino decoupling, the damping scale is $l_{\operatorname{dec}(v-\mathrm{e})}=\pi c t_{\mathrm{r}} r_{v} \frac{\widetilde{\Gamma}_{\operatorname{dec}(v-\mathrm{e})}}{H_{\mathrm{r}}} \sim 97 \mathrm{pc}$. The original calculation of this damping is due to Misner (1967). It corresponds to a mass scale somewhat below $1 M_{\odot}$. In the present case, the neutrino induced-damping scale takes this values in case the Dark Matter turns out to decouple from neutrinos just at the epoch the latter decouple from electrons.

Let us now give the value of the damping lengths for all regions of the neutrino parameter-space. Analytical expressions of these lengths may be found in Appendix D.2. Pending the astrophysical discussion in Paper II, we may already notice that the case $\phi_{\mathrm{dm}} \geq \phi_{v}$ makes little sense in case the relevant times to consider are close to the epoch of primordial nucleosynthesis, and is to be excluded. This is nevertheless possible somewhat before (provided annihilation occurs in time) or somewhat after (if Dark Matter particles are created late enough).

\subsubsection{Expressions of the neutrino-induced damping scales in Region A and B}

Region A:

$l_{v \mathrm{~d}}=\left.r_{v}\left(\frac{\Gamma_{\mathrm{dm}-v}}{\Gamma_{\nu-\mathrm{e}}}\right)^{\frac{1}{2}} \pi \frac{c t}{a}\right|_{\mathrm{dec}(\mathrm{dm}-v)}$,

or more explicitly

$l_{v \mathrm{~d}}=97 \mathrm{pc} r_{v} \kappa g_{*}^{\prime-\frac{3}{2}}\left(\frac{\widetilde{\Gamma}_{\mathrm{dm}-v}}{2.8 \times 10^{-30} \mathrm{~s}^{-1}}\right)^{\frac{5}{2}}$.

The largest value that this damping length can take is when $t_{\mathrm{dec}(\mathrm{dm}-v)}=t_{\mathrm{dec}(v-\mathrm{e})}$. This case only provides relevant limits if we require primordial fluctuations to exist down to very small scales of less than $100 \mathrm{pc}\left(\sim 0.1 M_{\odot}\right)$. It can be obtained from 


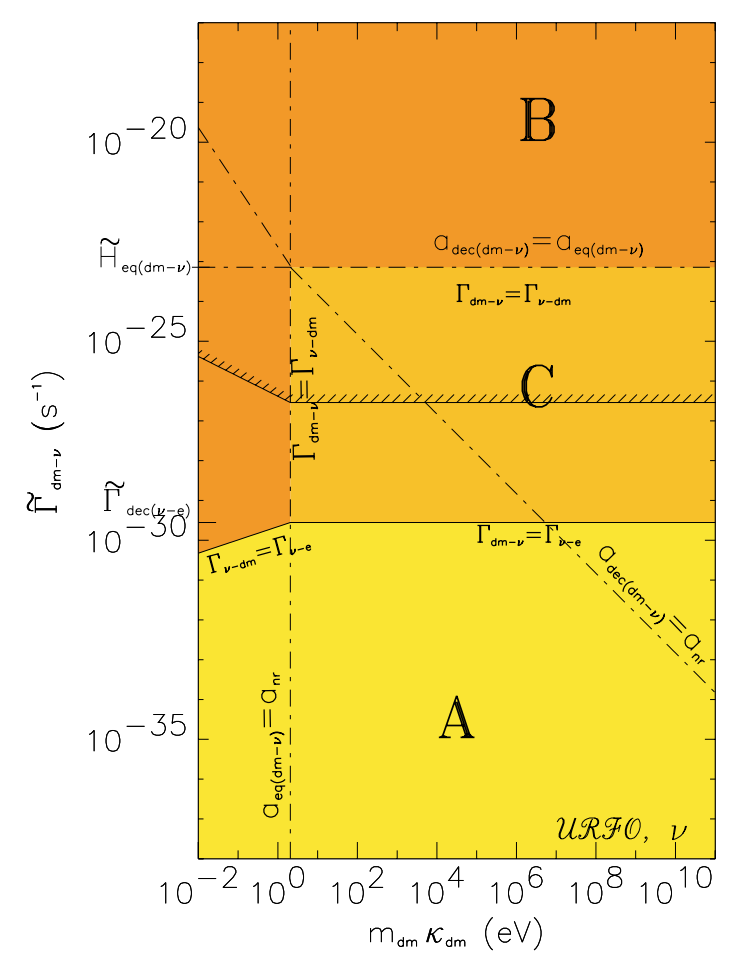

Fig. 2. Bounds in the (Dark Matter particles' mass, Dark Matter neutrino interaction rate) parameter space obtained from neutrino induced-damping in the URFO scenario. The Regions A, B and C are distinguished by different colors. They correspond to different expressions, given in the text, of the neutrino contribution to the Dark Matter damping length. The dot-dashed lines separate the domains according to the ordering of the epoch of the Dark Matter - neutrino decoupling, the non-relativistic transition and equality of the energy-densities. The hatches indicate in which part of the parameter space the neutrino induced-damping scale is greater than $100 \mathrm{kpc}\left(\sim 10^{8} M_{\odot}\right)$ scale. The indications in this figure are schematic. Some factors in general of order unity are omitted for simplicity. The reader interested by these constraints should use the expressions in the text, where all factors are given explicitly.

Eq. (75) by writing $l_{v \mathrm{~d}}<l_{\text {struct }}$ but will not be evaluated explicitly here as not of direct cosmological interest.

\section{Region B:}

$l_{v \mathrm{~d}}=\left.r_{v}\left(\frac{\Gamma_{\mathrm{dm}-v}}{\Gamma_{v-\mathrm{dm}}}\right)^{\frac{1}{2}} \pi \frac{c t}{a}\right|_{\mathrm{dec}(\mathrm{dm}-v)}$.

Only the case of a radiation-dominated universe is relevant here since the converse leads to prohibitive damping. Hence, as is readily seen in Figs. (2) to (5), we need to consider only the case where Dark Matter is relativistic $\left(a_{\operatorname{dec}(\mathrm{dm}-v)}<a_{\mathrm{nr}}\right)$. With the symmetry relations of the interaction rates (Sects. 2.4 and B.2), we get the explicit relations displayed in Tables 4 and 5. Note that we do not consider explicitly the NRFO scenario for $a_{\mathrm{eq}(\mathrm{dm}-v)}<a_{\mathrm{nr}}$, a case which is possible but quite unlikely for the reasons discussed above, see Sect. 4.2.6.

Also, for the neutrino interaction rates relevant to our calculation, decoupling of the Dark Matter with neutrinos is to occur well before the standard epoch of matter-radiation equality and the fate of the neutrinos afterwards is irrelevant to our

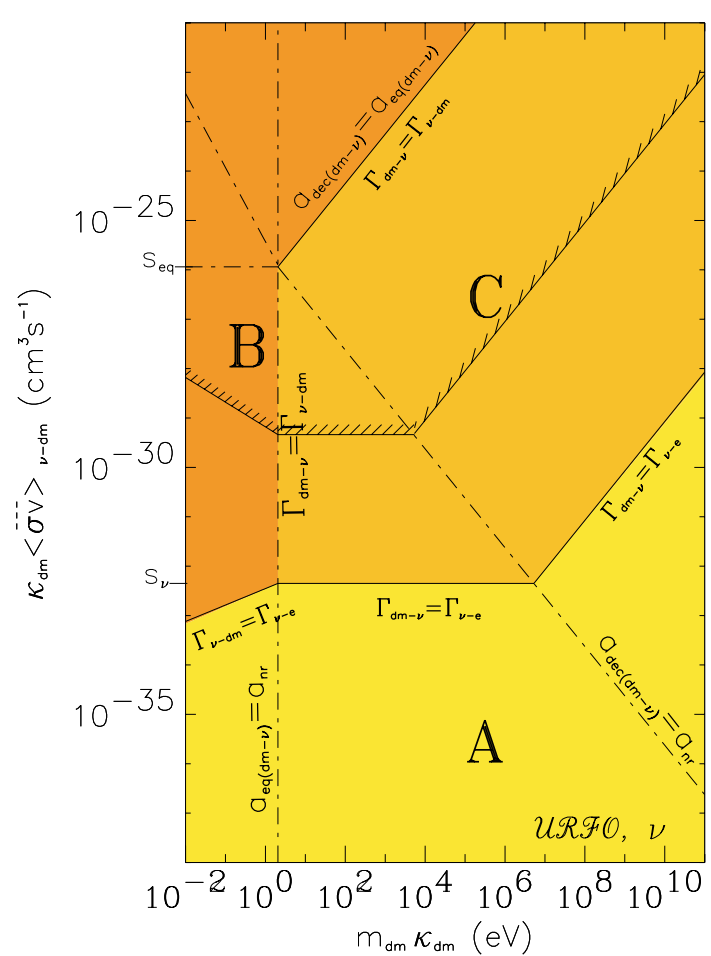

Fig. 3. Bounds in the (Dark Matter particles' mass, Dark Matter - neutrino cross-section) parameter space obtained from neutrino induceddamping in the URFO scenario. The Regions A, B and C are distinguished by different colorings. They correspond to different expressions, given in the text, of the neutrino contribution to the Dark Matter damping length. The dot-dashed lines separate the domains in the parameter space where the ordering of the Dark Matter - neutrino decoupling, the non-relativistic transition, the epoch of equality of the energy-densities changes. The hatches indicate the region in parameter space which is forbidden because the neutrino induced-damping yields damping above $100 \mathrm{kpc}\left(\sim 10^{8} M_{\odot}\right)$ scale. The indications in this figure are schematic. Some factors in general of order unity are omitted for simplicity. The reader interested by these constraints should use the expressions in the text, where all factors are given explicitly.

purpose: with the present limits on neutrino masses we remain well within the epoch where the neutrinos are fully relativistic.

\subsubsection{Expressions of the neutrino-induced damping scales in Region C}

The mixed-damping regime is at work in this region. We have (Eq. (44))

$\left.l_{v \mathrm{~d}} \sim \pi r_{v} \frac{c t}{a}\right|_{\operatorname{dec}(\mathrm{dm}-v)}$

Limits on $\widetilde{\Gamma}_{\mathrm{dm}-\gamma}$ are given in Tables 6 and 7, respectively, for the URFO and the NRFO scenario. These limits can again be transformed into limits for $\langle\overline{\sigma v}\rangle_{v-\mathrm{dm}}$ by means of the symmetry relations between the interaction rates (Sects. 2.4 and B.2).

This calculation does not require any knowledge of the number of electrons which are present, and holds whether the latter have annihilated or not. Also, all times of concern are well before the standard epoch of mater-radiation equality. It is worth to remember at this stage that our calculation are valid 


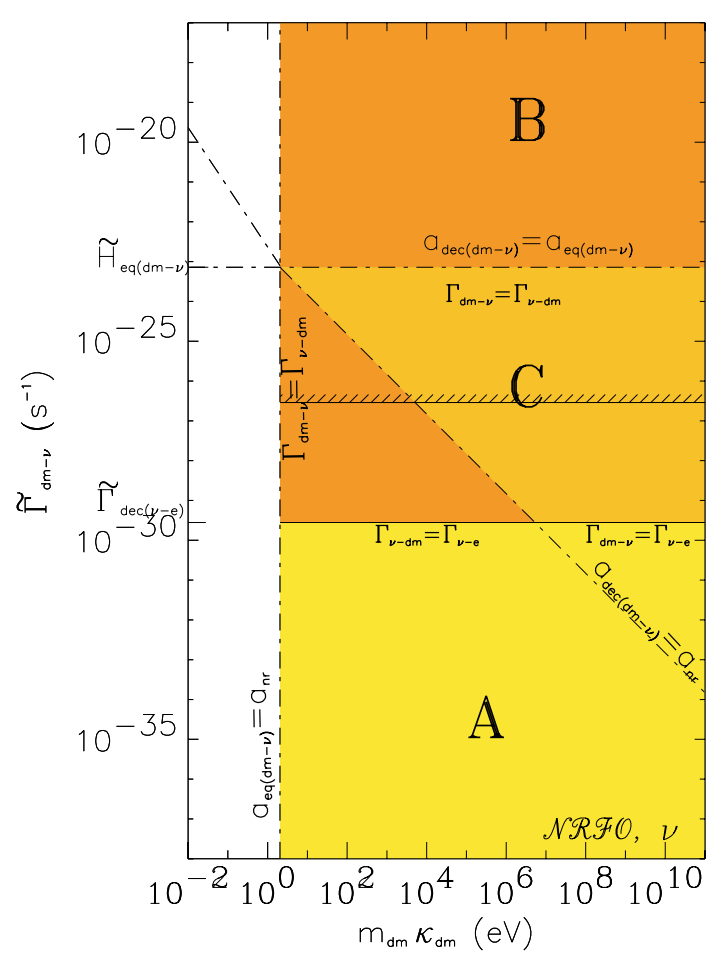

Fig. 4. Bounds in the (Dark Matter particles' mass, Dark Matter neutrino interaction rate) parameter space obtained from neutrino induced-damping in the NRFO scenario. The Regions A, B and C are distinguished by different colorings. They correspond to different expressions, given in the text, of the neutrino contribution to the Dark Matter damping length. The dot-dashed lines separate the domains in the parameter space where the ordering of the Dark Matter neutrino decoupling, the non-relativistic transition, the epoch of equality of the energy-densities changes. The hatches indicate the region in parameter space which is forbidden because the neutrino induceddamping yields damping above the $100 \mathrm{kpc}\left(\sim 10^{8} M_{\odot}\right)$ scale. The white area is not forbidden, but represents a case which is very unlikely to happen (see discussion in Sect. 4.2.6). The indications in this figure are schematic. Some factors in general of order unity are omitted for simplicity. The reader interested by these constraints should use the expressions in the text, where all factors are given explicitly.

for massive neutrinos, provided their mass is within the known physical and astrophysical limits.

\section{Constraining Dark Matter properties from the photon induced-damping scale}

As already mentioned, the possible coupling between Dark Matter and photons also induces a source of collisional damping. The associated damping scale $l_{\gamma \mathrm{d}}$ can be inferred from the formula (36). The calculation, however, is somewhat different from the one for $l_{v \mathrm{~d}}$ because the epoch at which the Universe becomes matter dominated and the epoch of the recombination now get into play. This introduces two new scales $\widetilde{H}_{\text {eq }(\mathrm{dm}-\gamma)}$ and $\widetilde{H}_{\text {rec }}$ which are the "reduced" $\left(\widetilde{H}=H a^{3}\right)$ Hubble rates at some specific time related to the epoch of equality and recombination, respectively (precise definition are given in Sect. A.3.3). Similarly to the neutrino case, from $\Gamma_{\gamma}=$ $\Gamma_{\gamma-\mathrm{e}}+\Gamma_{\gamma-\mathrm{dm}}+\ldots$ we see that the relevant interaction rates are

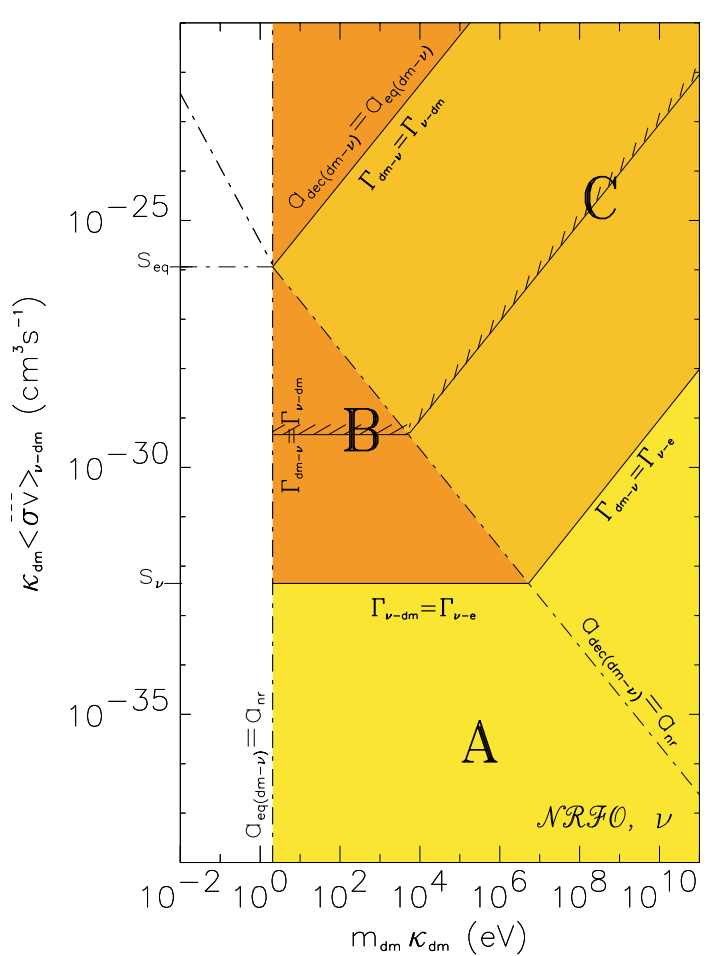

Fig. 5. Bounds in the (Dark Matter particles' mass, Dark Matter - neutrino cross-section) parameter space obtained from neutrino induceddamping in the NRFO scenario. The Regions A, B and C are distinguished by different colorings. They correspond to different expressions, given in the text, of the neutrino contribution to the Dark Matter damping length. The dot-dashed lines separate the domains in the parameter space where the ordering of the Dark Matter - neutrino decoupling, the non-relativistic transition, the epoch of equality of the energy-densities changes. The hatches indicate the region in parameter space which is forbidden because the neutrino induced-damping yields damping above the $100 \mathrm{kpc}\left(\sim 10^{8} M_{\odot}\right)$ scale. The white area is not forbidden, but represents a case which is very unlikely to happen (see discussion in Sect. 4.2.6). The indications in this figure are schematic. Some factors in general of order unity are omitted for simplicity. The reader interested by these constraints should use the expressions in the text, where all factors are given explicitly.

$\widetilde{\Gamma}_{\gamma}, \widetilde{\Gamma}_{\gamma-\mathrm{e}}$ and $\widetilde{\Gamma}_{\gamma-\mathrm{dm}}$ (see Sect. A.2.6). So, the parameter space for the photon induced-damping may be taken as $\left[m_{\mathrm{dm}}, \widetilde{\Gamma}_{\mathrm{dm}-\gamma}\right]$ or $\left[m_{\mathrm{dm}},\langle\overline{\sigma v}\rangle_{\gamma-\mathrm{dm}}\right]$.

\subsection{Dark Matter parameter space}

"Regions" are expected to appear, as in the neutrino case, depending on whether photons are collisional or collisionless. The new discussion here is about the role of recombination and the relevance of the epoch of matter dominance. The borderlines of these regions are given explicitely in Appendix C.3.

\subsubsection{Dark Matter decouples from photons while the latter are collisional}

This implies

$\widetilde{\Gamma}_{\mathrm{dm}-\gamma} \leq\left.\widetilde{\Gamma}_{\gamma}\right|_{\operatorname{dec}(\mathrm{dm}-\gamma)}$. 
Table 4. Region B (URFO scenario).

$$
\begin{array}{rl}
\hline \hline l_{v \mathrm{~d}} & 68 \mathrm{kpc} r_{v}\left(\frac{4 g^{\prime} * v}{3}\right)^{\frac{1}{2}} g_{*}^{\prime-1}\left(\frac{\Omega_{\mathrm{dm}} h_{70}{ }^{2}}{0.25}\right)^{-\frac{1}{2}} \\
& \left(\frac{m_{\mathrm{dm}} \kappa_{\mathrm{dm}}}{1 \mathrm{MeV}}\right)^{\frac{1}{2}} \frac{\widetilde{\Gamma}_{\mathrm{dm}-v}}{2.8 \times 10^{-30} \mathrm{~s}^{-1}} \\
\widetilde{\Gamma}_{\mathrm{dm}-v} & <4.1 \times 10^{-30} \mathrm{~s}^{-1} r_{v}^{-1}\left(\frac{4 g^{\prime}{ }_{* v}}{3}\right)^{-\frac{1}{2}} g^{\prime}{ }_{*} \\
& \left(\frac{\Omega_{\mathrm{dm}} h_{70}{ }^{2}}{0.25}\right)^{\frac{1}{2}}\left(\frac{m_{\mathrm{dm}} \kappa_{\mathrm{dm}}}{1 \mathrm{MeV}}\right)^{-\frac{1}{2}} \frac{l_{\mathrm{struct}}}{100 \mathrm{kpc}} \\
\kappa_{\mathrm{dm}}\langle\overline{\sigma v}\rangle_{v-\mathrm{dm}}<6.6 \times 10^{-33} \mathrm{~cm}^{3} \mathrm{~s}^{-1} r_{v}^{-1}\left(\frac{4 g^{\prime}{ }_{* v}}{3}\right)^{-\frac{3}{2}} g^{\prime}{ }_{*} \\
\\
\end{array}
$$

Table 5. Region B (NRFO scenario).

$$
\begin{array}{ll}
\hline \hline l_{v \mathrm{~d}} & =97 \mathrm{pc} r_{v}\left(\frac{g^{\prime}{ }_{* v}}{g^{\prime}{ }_{* \mathrm{dm}}}\right)^{\frac{1}{2}} g^{\prime-1} \frac{\widetilde{\Gamma}_{\mathrm{dm}-v}}{2.8 \times 10^{-30} \mathrm{~s}^{-1}} \\
\widetilde{\Gamma}_{\mathrm{dm}-v} & <2.9 \times 10^{-27} \mathrm{~s}^{-1} r_{v}^{-1}\left(\frac{g^{\prime}{ }_{* \mathrm{dm}}}{g^{\prime}{ }_{* v}}\right)^{\frac{1}{2}} g^{\prime}{ }_{*} \frac{l_{\text {struct }}}{100 \mathrm{kpc}} \\
\kappa_{\mathrm{dm}}\langle\overline{\sigma v}\rangle_{v-\mathrm{dm}}<4.6 \times 10^{-30} \mathrm{~cm}^{3} \mathrm{~s}^{-1} & <\left(\frac{4 g^{\prime}{ }_{* v}}{3}\right)^{-1}\left(\frac{g^{\prime}{ }_{* \mathrm{dm}}}{g^{\prime}{ }_{* v}}\right)^{\frac{1}{2}} g^{\prime} \frac{l_{\text {struct }}}{100 \mathrm{kpc}} \\
& r_{v}^{-1}
\end{array}
$$

Region A. Photons collisions dominated by photon-electron scattering.

$\Gamma_{\gamma-\mathrm{dm}} \leq\left.\Gamma_{\gamma-\mathrm{e}}\right|_{\mathrm{dec}(\mathrm{dm}-\gamma)}$

Region B. Photons collisions dominated by photonDark Matter scattering.

$\Gamma_{\gamma-\mathrm{dm}}>\left.\Gamma_{\gamma-\mathrm{e}}\right|_{\mathrm{dec}(\mathrm{dm}-\gamma)}$.

The separation between the two regions A and B may, among others, occur for $\mathrm{dm}-\gamma$ decoupling before recombination, that is for a sizeable $\Gamma_{\gamma-\mathrm{e}}$ rate. This requires simultaneously sufficiently small $\Gamma_{\mathrm{dm}-\gamma}$ rate for the decoupling be early enough, and a sufficiently large $\Gamma_{\gamma-\mathrm{dm}}$ rate to be larger than the $\gamma-\mathrm{e}$ interaction. These two, somewhat antagonistic, requirements can actually be satisfyed (see the evaluation in Appendix C.3), but for unrealistic values of the Dark Matter particles' mass $m_{\mathrm{dm}}$

\begin{tabular}{|c|c|}
\hline$l_{v \mathrm{~d}}$ & $\sim 97 \mathrm{pc} r_{v}{g^{\prime}}_{*}^{-1} \frac{\widetilde{\Gamma}_{\mathrm{dm}-v}}{2.8 \times 10^{-30} \mathrm{~s}^{-1}}$ \\
\hline$\widetilde{\Gamma}_{\mathrm{dm}-\nu}$ & $<2.9 \times 10^{-27} \mathrm{~s}^{-1} r_{v}^{-1} g^{\prime} * \frac{l_{\text {struct }}}{100 \mathrm{kpc}}$ \\
\hline$a_{\mathrm{dec}(\mathrm{dm}-v)}>a_{\mathrm{r}}$ & \\
\hline$\kappa_{\mathrm{dm}}\langle\overline{\sigma v}\rangle_{v-\mathrm{dm}}$ & $\begin{array}{l}<9.0 \times 10^{-28} \mathrm{~cm}^{3} \mathrm{~s}^{-1} \\
r_{v}^{-2}\left(\frac{4 g^{\prime}{ }_{* v}}{3}\right)^{-1} g_{*}^{\prime \frac{3}{2}} \frac{m_{\mathrm{dm}} \kappa_{\mathrm{dm}}}{1 \mathrm{MeV}}\left(\frac{l_{\text {struct }}}{100 \mathrm{kpc}}\right)^{2}\end{array}$ \\
\hline$a_{\mathrm{dec}(\mathrm{dm}-v)}<a_{\mathrm{r}}$ & \\
\hline$\kappa_{\mathrm{dm}}\langle\overline{\sigma v}\rangle_{v-\mathrm{dm}}$ & $\begin{array}{l}<4.6 \times 10^{-30} \mathrm{~cm}^{3} \mathrm{~s}^{-1} \\
r_{v}^{-1}\left(\frac{4 g^{\prime}{ }_{* v}}{3}\right)^{-1} g^{\prime}{ }_{*} \frac{l_{\text {struct }}}{100 \mathrm{kpc}}\end{array}$ \\
\hline
\end{tabular}
(of the order of $10^{-2} \mathrm{eV}$ ). This separation is barely visible in Figs. 6 and 7.

More realistically, the separation between the regions A and B simply occurs at recombination: before the latter the $\gamma-\mathrm{e}$ interactions dominate, and after, only $\gamma-\mathrm{dm}$ interactions are left. So, Regions A and B in practical cases correspond just to the separation between $\mathrm{dm}-\gamma$ decoupling before and after recombination (Table 8 ).
Table 6. Region C (URFO scenario).

Table 7. Region C (NRFO scenario).

\begin{tabular}{ll}
\hline \hline$l_{v \mathrm{~d}}$ & $\sim 97 \mathrm{pc} r_{v} g_{*}^{\prime-1} \frac{\widetilde{\Gamma}_{\mathrm{dm}-v}}{2.8 \times 10^{-30} \mathrm{~s}^{-1}}$ \\
$\widetilde{\Gamma}_{\mathrm{dm}-v}$ & $<2.9 \times 10^{-27} \mathrm{~s}^{-1} r_{v}^{-1} g^{\prime} * \frac{l_{\text {struct }}}{100 \mathrm{kpc}}$ \\
$a_{\mathrm{dec}(\mathrm{dm}-v)}>a_{\mathrm{nr}}$ & $<9.0 \times 10^{-28} \mathrm{~cm}^{3} \mathrm{~s}^{-1}$ \\
$\kappa_{\mathrm{dm}}\langle\overline{\sigma v}\rangle_{v-\mathrm{dm}}$ & $r_{v}^{-2}\left(\frac{4 g^{\prime} * v}{3}\right)^{-1} g^{\frac{3}{2}} \frac{m_{\mathrm{dm}} \kappa_{\mathrm{dm}}}{1 \mathrm{MeV}}\left(\frac{l_{\text {struct }}}{100 \mathrm{kpc}}\right)^{2}$ \\
$a_{\mathrm{dec}(\mathrm{dm}-v)}<a_{\mathrm{nr}}$ & \\
does not exist in this case
\end{tabular}

\subsubsection{Dark Matter decouples from photons while the latter are freely-propagating}

This is the analogue of region $\mathrm{C}$ in the neutrino case. It requires $\Gamma_{\gamma}<H$ at the Dark Matter - photon decoupling $\left(\Gamma_{\mathrm{dm}-\gamma}=H\right)$. The earliest this may happen, whatever the strength of the Dark Matter interactions, is at recombination: so, Dark Matter - photon decoupling necessarily occurs at an epoch where the universe is matter-dominated. One then has necessarily $\Gamma_{\mathrm{dm}-\gamma} \leq \Gamma_{\gamma-\mathrm{dm}}$ according to the relation (23). But the first relation implies $\Gamma_{\gamma-\mathrm{dm}}<H$ and in turn the second $\Gamma_{\mathrm{dm}-\gamma}<H$. Therefore Dark Matter cannot remain coupled to photons while the latter are freely propagating. So there is no Region C, i.e. no mixed-damping regime. 


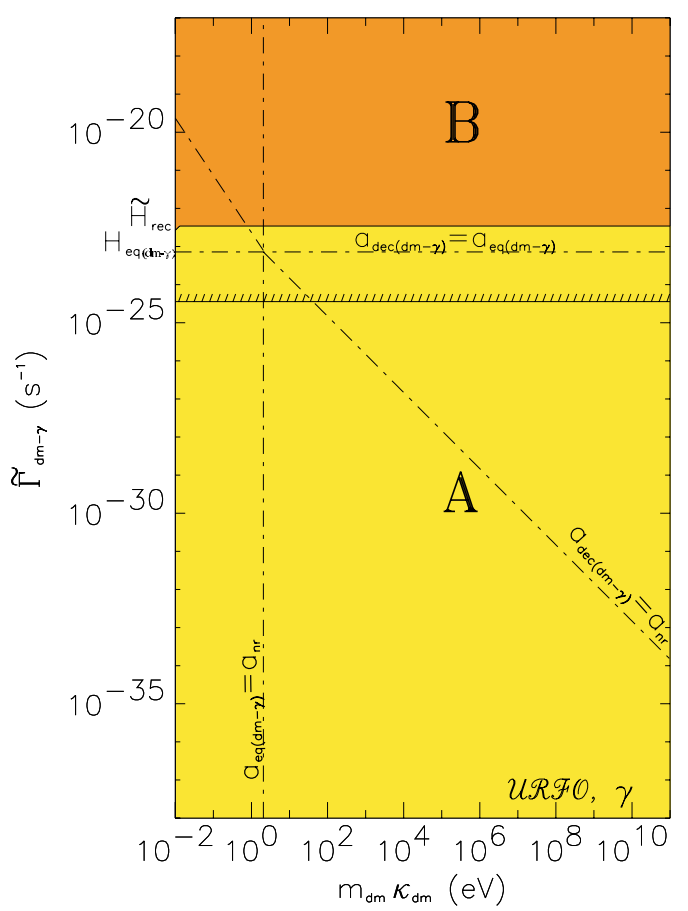

Fig. 6. Bounds in the (Dark Matter particles' mass, Dark Matter photon interaction rate) parameter space obtained from photon induced-damping in the URFO scenario. The Regions A and B are distinguished by different colorings. They correspond to different expressions, given in the text, of the photon contribution to the Dark Matter damping length. The dot-dashed lines separate the domains in the parameter space where the ordering of the Dark Matter - photon decoupling, the non-relativistic transition, the epoch of equality of the energy-densities changes. The hatches indicate the region in parameter space which is forbidden because the photon induced-damping yields damping above the $100 \mathrm{kpc}\left(\sim 10^{8} M_{\odot}\right)$ scale. The indications in this figure are schematic. Some factors in general of order unity are omitted for simplicity. The reader interested by these constraints should use the expressions in the text, where all factors are given explicitly.

\subsection{Photon induced-damping scales and limits on the interaction rates}

We now compute the damping scales associated with the regions $\mathrm{A}$ and $\mathrm{B}$ for the two scenarios NRFO and URFO. Region A is slightly more complicated than region B because it can potentially exist in both a radiation and matter dominated Universe while region B necessarily takes place in a matter dominated Universe. We give here the most relevant numerical results. A more systematic presentation of our analytical results is given in Appendix D.3.

\subsubsection{Expression of the photon-induced damping scale for Region A}

The condition $\widetilde{\Gamma}_{\gamma-\mathrm{e}}>\widetilde{\Gamma}_{\gamma-\mathrm{dm}}$ is in some sense the standard situation since, in this case, the photon decoupling is set by the recombination epoch. The damping of the Dark Matter fluctuations is then induced by the interaction of photons with the electrons. Below a temperature around $100 \mathrm{keV}$ (a case which is relevant for $\widetilde{\Gamma}_{\mathrm{dm}-\gamma}>10^{-28} \mathrm{~s}^{-1}$ and is the only regime of

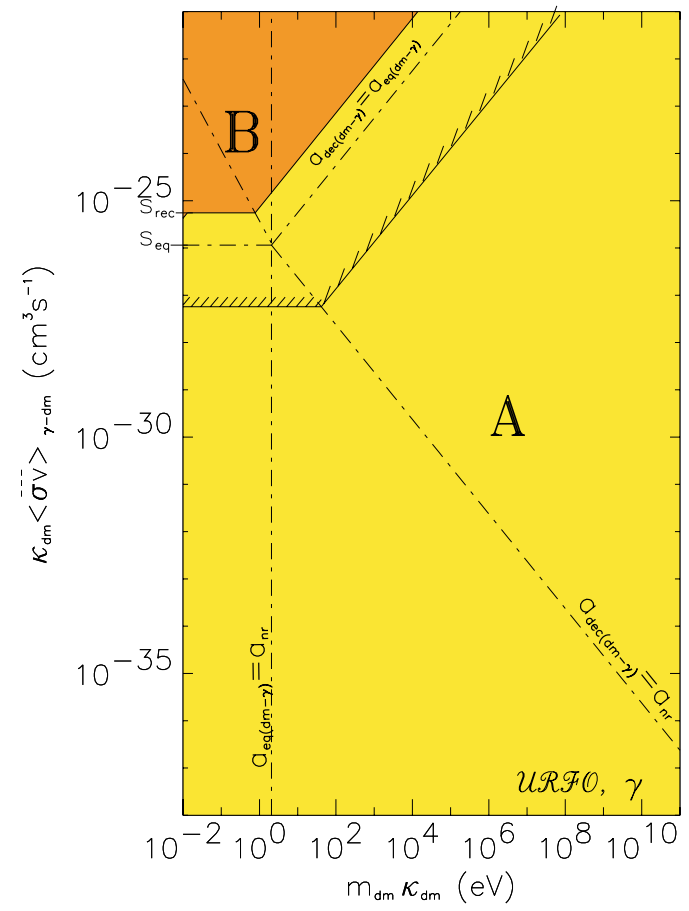

Fig. 7. Bounds in the (Dark particles' Matter mass, Dark Matter photon cross-section) parameter space obtained from photon induceddamping in the URFO scenario. The Regions A and B are distinguished by different colorings. They correspond to different expressions, given in the text, of the photon contribution to the Dark Matter damping length. The dot-dashed lines separate the domains in the parameter space where the ordering of the Dark Matter - photon decoupling, the non-relativistic transition, the epoch of equality of the energy-densities changes. The hatches indicate the region in parameter space which is forbidden because the photon induced-damping yields damping above the $100 \mathrm{kpc}\left(\sim 10^{8} M_{\odot}\right)$ scale. The indications in this figure are schematic. Some factors in general of order unity are omitted for simplicity. The reader interested by these constraints should use the expressions in the text, where all factors are given explicitly.

practical interest), the photon-electron elastic scattering crosssection (i.e. the Thomson cross-section) yields the reduced interaction rate $\widetilde{\Gamma}_{\mathrm{Th}}=\sigma_{\mathrm{Th}} c \widetilde{n}_{\mathrm{e}} \sim 5 \times 10^{-21} \mathrm{~s}^{-1}$, while the recombination corresponds to a (reduced) Hubble rate $\widetilde{H}_{\text {rec }} \sim$ $3 \times 10^{-23} \mathrm{~s}^{-1}$.

We get in this case

$l_{\gamma \mathrm{d}}=\left.\pi r_{\gamma}\left(\frac{\widetilde{\Gamma}_{\mathrm{dm}-\gamma}}{\widetilde{\Gamma}_{\mathrm{Th}}}\right)^{\frac{1}{2}} \frac{c t}{a}\right|_{\operatorname{dec}(\mathrm{dm}-\gamma)}$.

We are led to consider the two following cases:

1. Dark Matter decouples from photons in a radiation dominated Universe, that is $\widetilde{\Gamma}_{\mathrm{dm}-\gamma}<\widetilde{H}_{\mathrm{eq}(\mathrm{dm}-\gamma)}$. In this case the shear viscosity dominates. This yields the damping length as well as the constraints given in Table 9.

2. Dark Matter decouples from photons in a matter dominated Universe, that is $\widetilde{\Gamma}_{\mathrm{dm}-\gamma}>\widetilde{H}_{\mathrm{eq}(\mathrm{dm}-\gamma)}$. This is the case where 


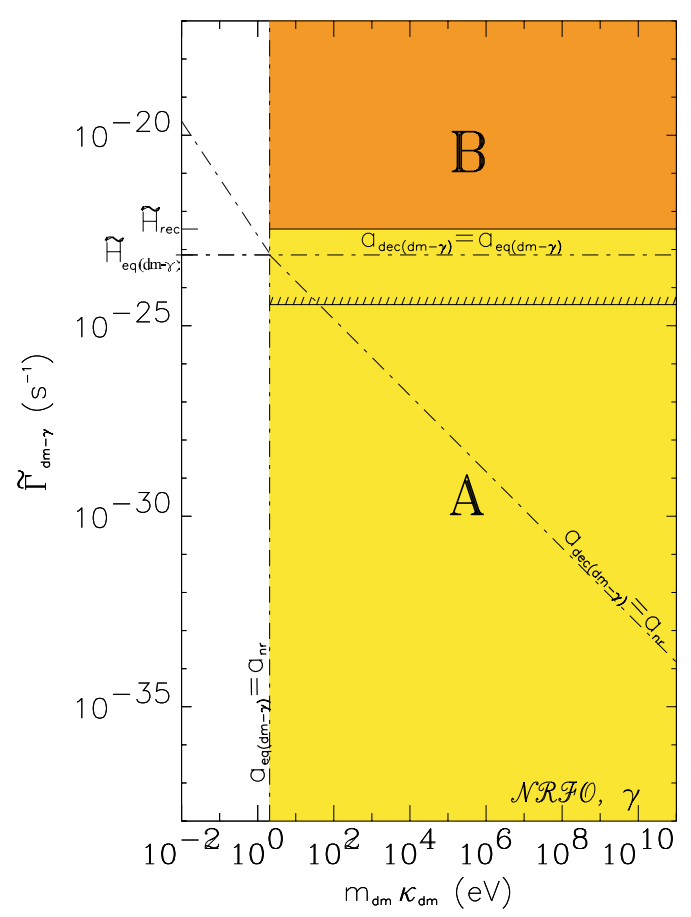

Fig. 8. Bounds in the (Dark Matter paticle's mass, Dark Matter photon interaction rate) parameter space obtained from photon induced-damping in the NRFO scenario. The Regions A and B are distinguished by different colorings. They correspond to different expressions, given in the text, of the photon contribution to the Dark Matter damping length. The dot-dashed lines separate the domains in the parameter space where the ordering of the Dark Matter - photon decoupling, the non-relativistic transition, the epoch of equality of the energy-densities changes. The hatches indicate the region in parameter space which is forbidden because the photon induced-damping yields damping above the $100 \mathrm{kpc}\left(\sim 10^{8} M_{\odot}\right)$ scale. The white area is not forbidden, but represents a case which is very unlikely to happen (see discussion in Sect. 4.2.6). The indications in this figure are schematic. Some factors in general of order unity are omitted for simplicity. The reader interested by these constraints should use the expressions in the text, where all factors are given explicitly.

Table 8. Definition of the two Dark Matter Regions useful for the photon induced-damping calculation.

\begin{tabular}{lll}
\hline \hline Region A & $\widetilde{\Gamma}_{\mathrm{dm}-\gamma} \leq\left.\widetilde{\Gamma}_{\gamma-\mathrm{e}}\right|_{\operatorname{dec}(\mathrm{dm}-\gamma)}$ & $a_{\mathrm{dec}(\mathrm{dm}-\gamma)} \leq a_{\mathrm{rec}}$ \\
Region B & $\widetilde{\Gamma}_{\mathrm{dm}-\gamma} \leq\left.\widetilde{\Gamma}_{\gamma-\mathrm{dm}}\right|_{\operatorname{dec}(\mathrm{dm}-\gamma)}$ & $a_{\mathrm{dec}(\mathrm{dm}-\gamma)}>a_{\mathrm{rec}}$ \\
\hline
\end{tabular}

the heat conduction dominates, so that

$$
\begin{aligned}
l_{\gamma \mathrm{d}}= & 8.2 \mathrm{Mpc} r_{\gamma}\left(\frac{\Omega_{\mathrm{m}} h_{70}{ }^{2}}{0.3}\right)^{\frac{1}{3}}\left(\frac{\Omega_{\mathrm{dm}} h_{70}{ }^{2}}{0.25}\right)^{-1} \\
& \times\left(\frac{\Omega_{\mathrm{b}} h_{70}{ }^{2}}{0.05}\right)^{-\frac{1}{2}}\left(\frac{\widetilde{\Gamma}_{\mathrm{dm}-\gamma}}{6 \times 10^{-24} \mathrm{~s}^{-1}}\right)^{\frac{5}{6}} .
\end{aligned}
$$

This scale is typically above $1 \mathrm{Mpc}$. So, when the Dark Matter - photon decoupling is in the matter dominated era, the photon induced-damping is always prohibitive according to our criteria.

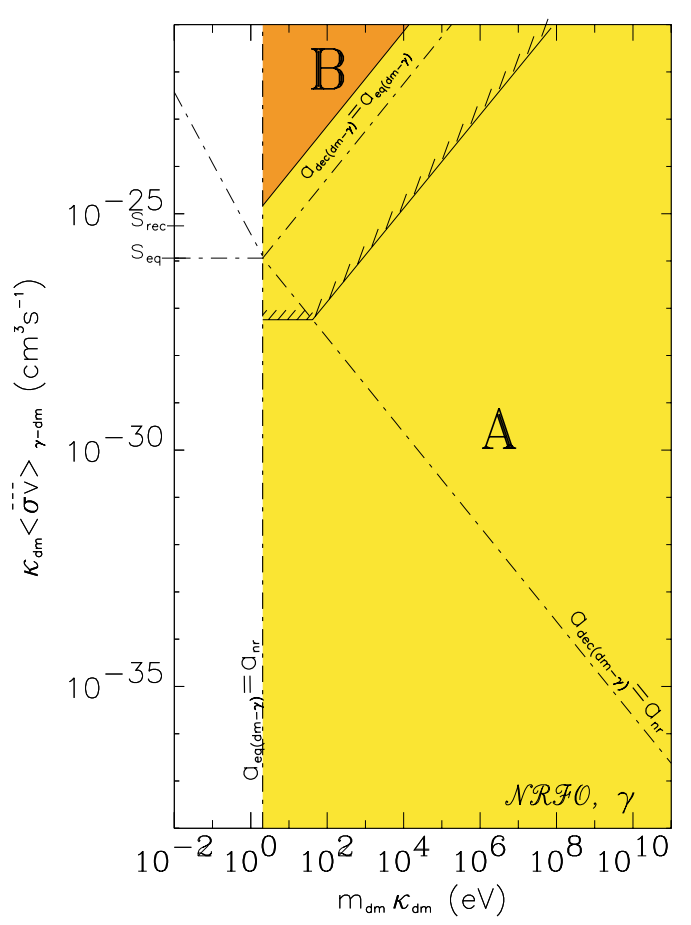

Fig. 9. Bounds in the (Dark Matter particles' mass, Dark Matter photon cross-section) parameter space obtained from photon induced-damping in the NRFO scenario. The Regions A and B are distinguished by different colorings. They correspond to different expressions, given in the text, of the photon contribution to the Dark Matter damping length. The dot-dashed lines separate the domains in the parameter space where the ordering of the Dark Matter - photon decoupling, the non-relativistic transition, the epoch of equality of the energy-densities changes. The hatches indicate the region in parameter space which is forbidden because the photon induced-damping yields damping above the $100 \mathrm{kpc}\left(\sim 10^{8} M_{\odot}\right)$ scale. The white area is not forbidden, but represents a case which is very unlikely to happen (see discussion in Sect. 4.2.6). The indications in this figure are schematic. Some factors in general of order unity are omitted for simplicity. The reader interested by these constraints should use the expressions in the text, where all factors are given explicitly.

It may be of interest to note that for the largest interaction rate allowed in Region $\mathrm{A}$, that is for $\widetilde{\Gamma}_{\mathrm{dm}-\gamma}=$ $\widetilde{H}_{\text {rec }}$, i.e. the Dark Matter decouples from the photons at the recombination epoch. The scale $l_{\gamma \mathrm{d}}$ thus reaches $l_{\mathrm{S}}=\pi c t_{\mathrm{m}} r_{\gamma}\left(\widetilde{H}_{\mathrm{rec}} a_{\mathrm{rec}} / \widetilde{\Gamma}_{\mathrm{Th}}\right)^{\frac{1}{2}}$, the Silk damping length which may be estimated as

$l_{\mathrm{S}}=4.9 \operatorname{Mpc}\left(\Omega_{\mathrm{m}} h_{70}^{2}\right)^{-\frac{1}{4}}\left(\Omega_{b} h_{70}^{2}\right)^{-\frac{1}{2}}$.

This length corresponds to a total mass (including the Dark Matter)

$M_{\mathrm{S}}=6.6 \times 10^{13} M_{\odot}\left(\Omega_{\mathrm{m}} h_{70}^{2}\right)^{\frac{1}{4}}\left(\Omega_{\mathrm{b}} h_{70}^{2}\right)^{-\frac{3}{2}}$.

It may be noted that, with the now well established values of $\Omega_{\mathrm{m}}, H_{0}$ and especially $\Omega_{\mathrm{b}}$, these are very large scales, of the order of $l_{\mathrm{S}}=30 \mathrm{Mpc}$ and $M_{\mathrm{S}}=10^{15} M_{\odot}$. 
Table 9. Region A. Decoupling in a radiation dominated Universe $\left(a_{\mathrm{dec}(\mathrm{dm}-\gamma)}<a_{\mathrm{eq}}\right)$.

$$
\begin{aligned}
& \overline{l_{\gamma \mathrm{d}}=8.2 \mathrm{Mpc} r_{\gamma} g_{*}^{\prime-1}\left(\frac{\Omega_{\mathrm{b}} h_{70}{ }^{2}}{0.05}\right)^{-\frac{1}{2}}\left(\frac{\widetilde{\Gamma}_{\mathrm{dm}-\gamma}}{6 \times 10^{-24} \mathrm{~s}^{-1}}\right)^{\frac{3}{2}}} \\
& \widetilde{\Gamma}_{\mathrm{dm}-\gamma}<3.2 \times 10^{-25} \mathrm{~s}^{-1} r_{\gamma}^{-\frac{2}{3}} g^{g_{*}^{2}}\left(\frac{\Omega_{\mathrm{b}} h_{70}{ }^{2}}{0.05}\right)^{\frac{1}{3}}\left(\frac{l_{\text {struct }}}{100 \mathrm{kpc}}\right)^{\frac{2}{3}} \\
& a_{\mathrm{dec}(\mathrm{dm}-\gamma)}>a_{\mathrm{nr}} \\
& \kappa_{\mathrm{dm}}\langle\overline{\sigma v}\rangle_{\gamma-\mathrm{dm}}<1.1 \times 10^{-23} \mathrm{~cm}^{3} \mathrm{~s}^{-1} r_{\gamma}^{-\frac{4}{3}}{g^{\prime}}_{*}^{\frac{5}{6}}(T) \\
& \left(\frac{\Omega_{\mathrm{b}} h_{70}{ }^{2}}{0.05}\right)^{\frac{2}{3}}\left(\frac{m_{\mathrm{dm}} \kappa_{\mathrm{dm}}}{1 \mathrm{MeV}}\right)\left(\frac{l_{\mathrm{struct}}}{100 \mathrm{kpc}}\right)^{\frac{4}{3}} \\
& a_{\mathrm{dec}(\mathrm{dm}-\gamma)}<a_{\mathrm{nr}} \\
& \kappa_{\mathrm{dm}}\langle\overline{\sigma v}\rangle_{\gamma-\mathrm{dm}}<5.1 \times 10^{-28} \mathrm{~cm}^{3} \mathrm{~s}^{-1} r_{\gamma}^{-\frac{2}{3}} g^{\prime}{ }_{*}^{\frac{2}{3}}\left(\frac{4 g^{\prime}{ }_{* \gamma}}{3}\right)^{-1} \\
& \left(\frac{\Omega_{\mathrm{b}} h_{70}{ }^{2}}{0.05}\right)^{\frac{1}{3}}\left(\frac{l_{\text {struct }}}{100 \mathrm{kpc}}\right)^{\frac{2}{3}}
\end{aligned}
$$

\subsubsection{Expression of the photon-induced damping scale for Region B}

The case where Dark Matter decouples from the photons after the recombination obviously leads to a damping which is larger than the Silk damping. We shortly treat this case for completeness. For cross-sections just above this threshold, that is for $\widetilde{\Gamma}_{\mathrm{dm}-\gamma} \sim \widetilde{H}_{\mathrm{rec}}$, the reduced photon - Dark Matter interaction rate is required to be $\widetilde{\Gamma}_{\gamma-\mathrm{dm}}=\widetilde{H}_{\mathrm{eq}(\mathrm{dm}-\gamma)}\left(\widetilde{H}_{\mathrm{rec}} / \widetilde{H}_{\mathrm{eq}(\mathrm{dm}-\gamma)}\right)^{5 / 3}$ and is smaller than $\widetilde{\Gamma}_{\mathrm{Th}}$. Since, after recombination, the photonelectron interactions become negligible, only $\widetilde{\Gamma}_{\gamma \text {-dm }}$ contributes to $\widetilde{\Gamma}_{\gamma}$. Such a photon - Dark Matter interaction therefore induces a sudden increase of the transparency (and of the viscosity coefficients), inducing in turn an increase of the damping length:

$l_{\gamma \mathrm{d}}=\left.\pi r_{\gamma}\left(\frac{\widetilde{\Gamma}_{\mathrm{dm}-\gamma}}{\widetilde{\Gamma}_{\gamma-\mathrm{dm}}}\right)^{1 / 2} \frac{c t}{a}\right|_{\operatorname{dec}(\mathrm{dm}-\gamma)}$.

More specifically, we obtain (for non-relativistic Dark Matter, as is the case in nearly all of Region B):

$l_{\gamma \mathrm{d}} \sim 350 \mathrm{Mpc} r_{\gamma}\left(\frac{\Omega_{\mathrm{m}} h_{70}{ }^{2}}{0.3}\right)^{-\frac{1}{2}}\left(\frac{\Omega_{\mathrm{dm}} h_{70}{ }^{2}}{0.25}\right)^{-\frac{1}{2}}$.

The damping length stays constant (and equal to the above value) whatever the interaction rate. For larger $d m-\gamma$ interaction rates, the viscosity coefficient gets smaller but the interaction occurs a longer time. The two effects happen to exactly compensate.

The damping in this case, obviously, is prohibitively large. This is also expected to be the case for the vanishingly small region in the parameter-space where the Dark Matter is relativistic.

\subsection{Limits on indirect Dark Matter - photon interactions}

Even neutral Dark Matter may experience photon induceddamping. Indeed, if the Dark Matter happens to be directly coupled to any of the species $j$ which are themselves coupled to the photon fluid (baryons, electrons etc.), the above damping is relevant. Photon induced-damping then is at work as long as the Dark Matter is coupled to the fluid $j$, and the previous estimates are relevant, with the replacement

$\widetilde{\Gamma}_{\mathrm{dm}-\gamma} \rightarrow \widetilde{\Gamma}_{\mathrm{dm}-j}=\langle\overline{\sigma v}\rangle_{\mathrm{dm}-j} \widetilde{n}_{j}$.

This can be used to get constraints on the relevant $\mathrm{dm}-j$ crosssections. We for instance get

$$
\begin{aligned}
\langle\overline{\sigma v}\rangle_{\mathrm{dm}-\mathrm{b}}< & 1.6 \times 10^{-18} \mathrm{~cm}^{3} \mathrm{~s}^{-1} r_{\gamma}^{-\frac{2}{3}} g^{\prime \frac{2}{3}}(T) \\
& \times\left(\frac{\Omega_{\mathrm{b}} h_{70}{ }^{2}}{0.05}\right)^{-\frac{2}{3}}\left(\frac{l_{\text {struct }}}{100 \mathrm{kpc}}\right)^{\frac{2}{3}}
\end{aligned}
$$

Exactly the same constraint holds for the dm - e cross-section.

\section{Conclusions}

In this general evaluation of damping properties, we have developed an approach which allows to discuss systematically all possible kinds of Dark Matter.

We have written the transport coefficients for a composite fluid of species in terms of the interaction rate of each of these species with the medium. This is rather straightforward for the shear viscosity and can be readily extended to heat conduction. We have taken some care to discuss bulk viscosity along the same lines, although the latter turns out for the sake of the present paper not to generate stronger constraints on the collision rates and cross-sections than shear viscosity. This provides a new insight into the physical processes at work, and has been used as an opportunity to correct some inaccuracies and wrong statements in earlier papers. We finally wrote all the transport coefficients of a composite fluid as a sum of contributions from the individual components, taking the coupling to the whole medium into account. This is the key step which allows us to discuss the damping of all possible kinds of Dark Matter.

We are led to separate, following Boehm et al. (2001), the contribution due to self-damping, that is the transport by the Dark Matter itself, and the one due to the induced-damping which is the transport by other species effective for damping the Dark Matter fluctuations. The classical examples of which being neutrinos and photons. This work aims to put our previous results on a general and firm basis.

Writing that each of these contributions alone must not produce prohibitive damping, we were able to get necessary conditions separately for the Dark Matter interaction rate with all coupled species, as well as for its specific interaction with each of the species it is coupled to. More generally, we identify the parameter space of relevant, namely interaction rate and 
Dark Matter particles' mass. Another parameter, which is seen to appear at the proper places, comes into play. The latter $\left(\kappa_{\mathrm{dm}}\right.$ in the text) measures the possible offset of the Dark Matter and photon temperatures. It is in practice of order unity or somewhat larger, but it must be stressed that in special circumstances (e.g. axion-like Dark Matter) this parameter may get overwhelmingly large and provide the dominant physics.

As expected, the bounds due to self-damping are closely related to the damping due to Dark Matter free-streaming. Both bear similar properties: the velocity of the Dark Matter particles, that is their mass, is the most important parameter. However, there are regions of parameter space where the Dark Matter interaction plays a role. As a result, in these regions, the expression of the free-streaming length (expressed in term of the Dark Matter mass), which is generally used for WDM candidates, does not hold anymore. Instead the freestreaming length depends on both the mass and interaction rate of the Dark Matter candidate. Said differently, the well-known requirement of having Dark Matter particles with a mass above $\sim 1 \mathrm{keV}$ is valid only in a restricted region of parameter space, which covers only a fraction of the Dark Matter models, where the Dark Matter interactions are really negligible. In regions where the Dark Matter interaction rate is not too large, the smallest acceptable value of the Dark Matter mass is above $1 \mathrm{MeV}$. In other parts, where the interaction rate turns out to be very large, extremely small masses (notably in the eV range) are allowed.

The bound due to the damping induced by a given species merely depends on the interaction rate of the Dark Matter with this species, as well as on the species' particle velocity. Relativistic species are the more efficient, damping the Dark Matter fluctuations nearly over the horizon size.

The neutrino induced-damping yields quite a rich parameter-space diagram (Dark-Matter-neutrino interaction rate/Dark Matter particles' mass). The bounds due to the possible coupling of the Dark Matter to the neutrinos turn out to be quite novel. Indeed, one in particular refers to Dark Matter that is coupled to neutrinos which are already free-streaming! We have evaluated analytically this effect in what we have called the mixed-damping. The difference with the collisional bound, which is only a lower bound to the damping length but takes only into account the effect of standard collisions, is large. Our mixed-damping estimate is still rather rough, although quite natural a result. It definitely would deserve a check by numerical simulations. The difference with the collisional bound is so large that there should be a substantial effect even in a realistic calculation, and the damping of a collisional fluid coupled to the free-streaming neutrinos should be the dominant contribution as we have stressed here. This is important, especially in the context where Warm Dark Matter scenarios. Indeed, we saw that, when our constraints are marginally satisfied, the damping induced by the neutrinos could yield a collisional Dark Matter candidate.

The bound due to a possible coupling of Dark Matter with photons (this may be the case through higher order reactions involving charged particles even if the Dark Matter is neutral), in the (Dark-Matter-photon interaction rate/ Dark Matter particles' mass) parameter space, are somewhat less constraining.
They are nevertheless relevant for a large fraction of the parameter space. Again, the possibility for Warm Dark Matter scenarios, where the damping is not due to free-streaming, but to interactions, exists.

The major (and nearly the only) hypothesis is that the Dark Matter went through a phase of statistical equilibrium, at some stage of its evolution. There is also the (milder) assumption that a sudden decrease by many orders of magnitude in the Dark Matter particle density is not possible elsewhere than at its non-relativistic transition (for instance a phenomenon like the recombination for the electrons - where the latter are substracted from the medium at a temperature bearing no relation with the electron mass - is assumed not to occur for Dark Matter).

Our bounds on the Dark Matter interactions with the most relevant species have been calculated without any assumption on the form of the primordial spectrum. The calculations are valid for any Dark Matter interaction rate and any Dark Matter particle mass, the values of practical interest covering 20 orders of magnitude for the interaction rates or cross-sections and much over 13 orders of magnitude for the Dark Matter particles' mass. Our estimates are valid within factors of order unity, a procedure which provides important simplifications in the classification. A thorough discussion of all possibilities allows to place any scenario within the relevant parameter space, with a precise statement on the important parameters and the actual physical process at work. The reader interested in a specific scenario can easily work out the relevant bound with more accuracy. He/she will find here a useful estimate of the result and a guide on how to proceed. The present results provide, we believe, a new starting basis for a systematic discussion on the possible nature of Dark Matter. The astrophysical relevance of these findings, including other constraints than just the damping condition, or more restrictive assumptions on the initial conditions, in particular those of (Boehm et al. 2002) which specifically restrict themselves to CDM initial conditions in order to yield more stringent limits, will be thoroughly discussed in Paper II.

Acknowledgements. The authors would like to thank P. Fayet for stimulating discussions.

\section{References}

Balian, R. 1992, From Microphysics to Macrophysics: Methods and Applications of Statistical Physics (Heidelberg, Springer)

Barkana, R., Haiman, Z., \& Ostriker, J. P. 2001

[arXiv: astro-ph/0102304]

Bautz, M. W., Arabadjis, J. S., \& Garmire, G. P. 2002

[arXiv:astro-ph/0202338]

Bernstein, J. 1988, Kinetic theory in the expanding universe (Cambridge and New York: Cambridge University Press)

Binney, J. J., \& Evans, N. W. 2001, MNRAS, 327, L27

Blumenthal, G. R., Pagels, H., \& Primack, J. R. 1982, Nature, 299, 37

Boehm, C., Fayet, P., \& Schaeffer, R. 2001, Phys. Lett. B, 518, 8

Boehm, C., Riazuelo, A., Hansen, S. H., \& Schaeffer, R. 2002, Phys. Rev. D, 66, 083505

Bond, J. R., Efstathiou, G., \& Silk, J. 1980, Phys. Rev. Lett., 45, 1980

Bond, J. R., \& Szalay, A. S. 1983, ApJ, 274, 443 
Chapman, S., \& Cowling, T. G. 1970, The mathematical theory of non-uniform gases: an account of the kinetic theory of viscosity, thermal conduction and diffusion in gases (Cambridge University Press), 3rd ed.

Chen, X.-1., Hannestad, S., \& Scherrer, R. J. 2002, Phys. Rev. D, 65, 123515

Chiu, W. A., Gnedin, N. Y., \& Ostriker, J. P. 2001

[arXiv:astro-ph/0103359]

Davis, M., Lecar, M., Pryor, C., \& Witten, E. 1981, ApJ, 250, 423

Dekel, A., \& Devor, J. 2003, MNRAS, 341, 326

Efstathiou, G., \& Silk, J. 1983, Fund. Cosmic Phys., 9, 1

Griest, K. 1988, Phys. Rev. D, 38, 2357

Gunn, J. E., Lee, B. W., Lerche, I., Schramm, D. N., \& Steigman, G. 1978, ApJ, 223, 1015

Haensel, P., \& Schaeffer, R. 1992, Phys. Rev. D, 45, 4708

Hu, W. T. 1995, Ph.D. Thesis [arXiv: astro-ph/9508126]

Hu, W. T., \& White, M. J. 1997, Phys. Rev., D56, 596

Israel, W., \& Stewart, J. M. 1979, Ann. Phys., 118, 341

Jou, D., Casas-Vazquez, J., \& Lebon, G. 2001, Extended irreversible thermodynamics (Springer, Heidelberg), 3rd ed.

Knebe, A., Devriendt, J., Mahmood, A., \& Silk, J. 2002, MNRAS, 329,813

Kolb, E. W., \& Turner, M. S. 1990, The early universe (Reading, MA, Addison-Wesley)

Lee, B. W., \& Weinberg, S. 1977, Phys. Rev. Lett., 39, 165

Ma, C.-P., \& Bertschinger, E. 1995, ApJ, 455, 7

Maartens, R. 1996, Causal thermodynamics in relativity, Lectures given at the Hanno Rund Worksop, South Africa [arXiv:astro-ph/9609119]

Misner, C. W. 1968, ApJ, 151, 431
Moore, B. 1994, Nature, 370, 629

Moore, B., Governato, F., Quinn, T., Stadel, J., \& Lake, G. 1998, ApJ, 499, L5

Moore, B., Ghigna, S., Governato, F., et al. 1999, ApJ, 524, L19

Peebles, P. J. E. 1980, The large-scale structure of the universe (Princeton, NJ: Princeton University Press)

Peebles, P. J. E. 1982, ApJ, 263, L1

Peebles, P. J. E., \& Yu, J. T. 1970, ApJ, 162, 815

Prada, F., Klypin, A., Flix, J., Martinez, M., \& Simonneau, E. 2004 [arXiv: astro-ph/0401512]

Schaeffer, R., \& Silk, J. 1985, ApJ, 292, 319

Schaeffer, R., \& Silk, J. 1988, ApJ, 332, 1

Silk, J. 1968a, ApJ, 151, L19

Silk, J. I. 1968b, Ph.D. Thesis

Sommer-Larsen, J., \& Dolgov, A. 1999 [arXiv: astro-ph/9912166]

Spergel, D. N., \& Steinhardt, P. J. 2000, Phys. Rev. Lett., 84, 3760 Spergel, D. N., Verde, L., Peiris, H. V., et al. 2003, ApJS, 148, 175

Stoehr, F., White, S. D. M., Tormen, G., \& Springel, V. 2002, MNRAS, 335, L84

van den Bosch, F. C., Robertson, B. E., Dalcanton, J. J., \& de Blok, W. J. G. 1999 [arXiv: astro-ph/9911372]

van den Bosch, F. C., \& Swaters, R. A. 2001, MNRAS, 325, 1017

Weinberg, S. 1971, ApJ, 168, 175

Yoshida, N., Sokasian, A., Hernquist, L., \& Springel, V. 2003, ApJ, 598,73

Yoshida, N., Springel, V., White, S. D. M., \& Tormen, G. 2000, ApJ, 544, L87

Zimdahl, W. 1996, MNRAS, 280, 1239 


\section{Online Material}




\section{Appendix A: Definitions and notations}

We give here the expressions actually used in the text. They may be obtained from elementary textbooks (e.g. Kolb \& Turner 1990).

\section{A.1. Present-day Universe}

We write the present-day Hubble constant as

$H_{0}=70 h_{70} \mathrm{~km} \mathrm{~s}^{-1} \mathrm{Mpc}^{-1}$.

\section{A.1.1. Temperature}

We use for the present CMB temperature the value

$T_{0}=2.73 \mathrm{~K}=2.35 \times 10^{-10} \mathrm{MeV}$,

that is

$\frac{\hbar c}{T_{0}}=8.39 \times 10^{-2} \mathrm{~cm}$

\section{A.1.2. Energy densities}

The present critical, photon and neutrino (the latter for our "reference" cosmology) energy densities are

$$
\begin{aligned}
\rho_{0 c} & =9.21 \times 10^{-30} h_{70}{ }^{2} \mathrm{~g} \mathrm{~cm}^{-3} \\
& =5.16 \times 10^{-3} h_{70}{ }^{2} \mathrm{MeV} / \mathrm{c}^{2} \mathrm{~cm}^{-3} \\
& =1.36 \times 10^{11} h_{70}{ }^{2} M_{\odot} \mathrm{Mpc}^{-3}
\end{aligned}
$$

$\rho_{0 \gamma}=4.67 \times 10^{-34} \mathrm{~g} \mathrm{~cm}^{-3}$

$\rho_{0 v \text { ref }}=3.19 \times 10^{-34} \mathrm{~g} \mathrm{~cm}^{-3}$

$\rho_{0(\gamma+v \text { ref })}=7.8610^{-34} \mathrm{~g} \mathrm{~cm}^{-3}$.

The ratio of the total matter, Dark Matter and baryon energy densities to the critical density are denoted respectively $\Omega_{\mathrm{m}}$, $\Omega_{\mathrm{dm}}$ and $\Omega_{\mathrm{b}}$ :

$$
\begin{aligned}
& \rho_{0 \mathrm{~m}}=\Omega_{\mathrm{m}} \rho_{0 \mathrm{c}} \\
& \rho_{0 \mathrm{dm}}=\Omega_{\mathrm{dm}} \rho_{0 \mathrm{c}} \\
& \rho_{0 \mathrm{~b}}=\Omega_{\mathrm{b}} \rho_{0 \mathrm{c}} .
\end{aligned}
$$

\section{A.1.3. Number densities}

The Dark Matter, photon, neutrino (the latter in our "reference" cosmology), and baryon number densities are respectively equal to:

$n_{0 \gamma}=413 \mathrm{~cm}^{-3}$

$n_{0 v \text { ref }}=338 \mathrm{~cm}^{-3}$

$n_{0 \mathrm{dm}}=1.29 \times 10^{-3} \mathrm{~cm}^{-3} \frac{\Omega_{\mathrm{dm}} h_{70}^{2}}{0.25}\left(\frac{m_{\mathrm{dm}}}{1 \mathrm{MeV}}\right)^{-1}$

$n_{0 \mathrm{~b}}=2.75 \times 10^{-7} \mathrm{~cm}^{-3} \frac{\Omega_{\mathrm{b}} h_{70}{ }^{2}}{0.05}$.

The latter value has been obtained assuming an average mass per baryon of $938 \mathrm{MeV}$, that is the proton mass. It will also be used as the comoving number of baryons since we neglect in this paper the slight average mass offset, upwards before nucleosynthesis, and downwards after.

\section{A.2. Evolution}

\section{A.2.1. Times}

We write the scale factor as

$a=\left(\frac{t}{t_{\alpha}}\right)^{\alpha}$,

This expression holds even if $\Omega$ is not unity, and/or $\Lambda$ nonzero provided the scale factor $a$ is not too close to unity, say for $a<\frac{1}{5}$, if $\Omega$ is not much smaller than 0.2 , and $\Lambda$ not much larger than 1 . The parameter $\alpha$ is equal to $1 / 2$ in the radiation dominated era and equal to $2 / 3$ in the matter dominated era. The normalization $t_{\alpha}$ may be obtained by matching the above expression to the Friedman equations. In the radiation dominated era, $t_{\alpha}$ is equal to

$$
\begin{aligned}
& t_{r} \equiv t_{1 / 2}=\left[\frac{16 \pi G}{3} \frac{g_{*}(T)}{\kappa^{4}(T)} \rho_{\gamma}\left(T_{0}\right)\right]^{-\frac{1}{2}} \\
& =2.39 \times 10^{19} \mathrm{~s}^{\prime-\frac{1}{2}}(T) \\
& =2.32 \times 10^{5} \mathrm{Mpc} / \mathrm{c} \mathrm{g}^{\prime-\frac{1}{2}}(T)
\end{aligned}
$$

where (see Sect. A.2.4 for more detail) $g^{\prime}{ }_{*}=g_{*} / 3.36$. In the matter dominated era, $t_{\alpha}$ is equal to

$$
\begin{aligned}
t_{\mathrm{m}} \equiv t_{2 / 3} & =\left[6 \pi G \rho_{\mathrm{m}}\left(T_{0}\right)\right]^{-\frac{1}{2}} \\
& =5.37 \times 10^{17} \mathrm{~s}\left(\frac{\Omega_{\mathrm{m}} h_{70}{ }^{2}}{0.3}\right)^{-\frac{1}{2}} \\
& =5.21 \times 10^{3} \mathrm{Mpc} / c\left(\frac{\Omega_{\mathrm{m}} h_{70}{ }^{2}}{0.3}\right)^{-\frac{1}{2}} .
\end{aligned}
$$

At the epoch of equality of matter and radiation, the expansion factor is not a power-law and the above approximations break down. They have been established so as to be exact far from this point. For this reason, it is slightly inaccurate to define the time of equality by equating the two expressions of the expansion parameter one obtains for $\alpha=1 / 2$ and $\alpha=2 / 3$. A better way of defining the epoch of equality in terms of the expansion factor $a_{\text {eq }}$ will be given in the following section, equating the associated Hubble rates which are more directly connected with the energy densities of the various species. With the definition of $a_{\text {eq }}$ given in Sect. A.2.2, we have

$t_{\mathrm{m}} \propto t_{\mathrm{r}} a_{\mathrm{eq}}^{\frac{1}{2}}$.

\section{A.2.2. Hubble rates}

We introduce the constant

$H_{\alpha}=\frac{\alpha}{t_{\alpha}}$.

The latter is in the radiation dominated era equal to

$H_{\mathrm{r}} \equiv H_{1 / 2}=2.10 \times 10^{-20} \mathrm{~s}^{-1}{g^{\prime}}^{\frac{1}{2}}(T)$

and in the matter dominated era equal to

$H_{\mathrm{m}} \equiv H_{2 / 3}=1.24 \times 10^{-18} \mathrm{~s}^{-1}\left(\frac{\Omega_{\mathrm{m}} h_{70}{ }^{2}}{0.3}\right)^{\frac{1}{2}}$. 


\section{Boehm and R. Schaeffer: Dark Matter damping lengths, Online Material p 3}

This allows us to write the relation between the expansion factor and the Hubble parameter at a given epoch as

$a=\left(\frac{H}{H_{\alpha}}\right)^{-\alpha}$.

It will also be convenient, for further use, to define a "reduced" Hubble rate

$\widetilde{H}=H a^{3}$.

Hence we have also

$a=\left(\frac{\widetilde{H}}{H_{\alpha}}\right)^{\frac{\alpha}{3 \alpha-1}}$.

The epoch of matter-radiation equality will be defined by equating the two expressions of $a$ obtained for $\alpha=1 / 2$ and $\alpha=2 / 3$, so that the Hubble rate, which is proportional via the Friedman equations to the energy-densities, is continuous at the transition, reflecting the continuity of the energy densities. We then get at the epoch of equality

$H=H_{\mathrm{eq}} \equiv \frac{H_{\mathrm{m}}^{4}}{H_{\mathrm{r}}^{3}}=2.59 \times 10^{-13} \mathrm{~s}^{-1}{g^{\prime}}_{*}^{-\frac{3}{2}}\left(T_{\mathrm{eq}}\right)\left(\frac{\Omega_{\mathrm{m}} h_{70}{ }^{2}}{0.3}\right)^{2}$

and

$\widetilde{H}=\widetilde{H}_{\mathrm{eq}} \equiv \frac{H_{\mathrm{r}}^{3}}{H_{\mathrm{m}}^{2}}=5.96 \times 10^{-24} \mathrm{~s}^{-1}{g^{\prime}}_{*}^{\frac{3}{2}}\left(T_{\mathrm{eq}}\right)\left(\frac{\Omega_{\mathrm{m}} h_{70}{ }^{2}}{0.3}\right)^{-1}$.

This implies

$a_{\text {eq }}=\left(\frac{H_{\mathrm{r}}}{H_{\mathrm{m}}}\right)^{2}$

or, equivalently

$H_{\mathrm{r}}=H_{\mathrm{m}} a_{\mathrm{eq}}^{\frac{1}{2}}$.

\section{A.2.3. Temperatures}

The temperature of a given species $i$ with a thermal distribution ${ }^{10}$ will be denoted $T_{i}$. We omit the index $i$ when dealing with photons. For each species $i$, we may define a parameter $\kappa_{i}(T)$ which relates the temperature $T_{i}$ of the species $i$ to the scale-factor $a$ :

$T_{i}=\frac{T_{0}}{\kappa_{i}(T) a}$.

This parameter $\kappa_{i}$ is constant in most cases. Its value at a given time depends on the history of the system. It can be determined by using the comoving entropy conservation when the latter is indeed conserved. As is well-known, the term $\kappa_{i}$ changes if, for instance, the fluid to which $i$ belongs contains another species which is under way of annihilating. We use $\kappa_{\gamma}(T) \equiv \kappa(T)$. The latter is equal to unity nowadays: $\kappa\left(T_{0}\right)=1$, while $\kappa_{\nu}\left(T_{0}\right)$, which is associated with the present neutrino temperature, is, in case our "reference" cosmology is adopted, equal to $(11 / 4)^{\frac{1}{3}}$.

\footnotetext{
${ }^{10}$ Even if the distribution is non-thermal, one can define by averaging over the actual distribution an effective temperature $T_{i}=<\frac{p^{2}}{3 e}>$, and accordingly a parameter $\kappa_{i}(T)$, which for most if not all applications in the present paper, play the same role.
}

\section{A.2.4. Energy densities}

The energy density of a relativistic species is given by:

$\rho_{i}=\epsilon \frac{g_{i}}{2} a_{\mathrm{SB}} T_{i}^{4}$,

with $a_{\mathrm{SB}}$ is the Stephan-Boltzmann constant, $g_{i}$ the spindegeneracy factor and $\epsilon$ a factor equal to $7 / 8$ or 1 for, respectively, Fermi or Bose-Einstein statistics of relativistic particles with negligible chemical potential ${ }^{11}$.

It turns out to be convenient to write the previous relation as

$\rho_{i}=\frac{g_{* i}}{2} a_{\mathrm{SB}} T^{4}$

where

$g_{* i}=\epsilon g_{i} \frac{\kappa^{4}}{\kappa_{i}^{4}}$.

We also define the quantity

$g_{* i}^{\prime}(T)=\frac{g_{* i}(T)}{3.36 \kappa^{4}(T)}$,

which is more useful than $g_{* i}$ for the calculations of the damping scales. This yields

$\rho_{i}=g^{\prime}{ }_{* i}(T) \frac{\rho_{0(\gamma+v \text { ref })}}{a^{4}}$

The parameter $g^{\prime}{ }_{* i}$ is, under normal circumstances, of the order of unity. We have, at the present epoch for our "reference" cosmology, $g_{* v \text { ref }}^{\prime}=0.41$ and $g_{* \gamma \text { ref }}^{\prime}=0.59$.

We also have, summing over all relativistic species in the universe

$g_{*}^{\prime}(T)=\sum_{i} g_{* i}^{\prime}(T)=\frac{g_{*}(T)}{3.36 \kappa^{4}(T)}$,

so as to get

$\rho_{\mathrm{r}}=g^{\prime}{ }_{*}(T) \frac{\rho_{0(\gamma+v \mathrm{ref})}}{a^{4}}$.

In our "reference" cosmology, this factor is unity after the electron annihilation. At early times, $g^{\prime}{ }_{*}(T)$ behaves as $1.9 g_{*}^{-1 / 3}(T)$.

Finally, we define

$\phi_{i}=\rho_{i}+p_{i}$

where $p_{i}$ is the pressure of species $i$. For relativistic matter, $\phi_{i} \sim \frac{4}{3} \rho_{i}$ while for non-relativistic matter $\phi_{i} \sim \rho_{i}$.

11 The factor $\epsilon$ may differ from $7 / 8$ or unity if we consider, for instance, relativistic particles with non-zero chemical potential (as may be the case if they are chemically decoupled but still in thermal equilibrium). 


\section{Boehm and R. Schaeffer: Dark Matter damping lengths, Online Material p 4}

\section{A.2.5. Number density}

We use throughout this paper comoving number densities, namely:

$\widetilde{n}=n a^{3}$

The latter appear more convenient because they are constant as a function of time when the particle number is conserved, so we keep only the dependence due to the microphysics, the cosmological dependence being removed. In particular, the comoving number density of protons or electrons before or after their non relativistic transition, are respectively

$\widetilde{n}_{\mathrm{p}}\left(T_{\mathrm{b}}>m_{\mathrm{p}} / 3\right)=\widetilde{n}_{\mathrm{e}}\left(T_{\mathrm{e}}>m_{\mathrm{e}} / 3\right)$,

$\widetilde{n}_{\mathrm{b}}\left(T_{\mathrm{b}}<m_{\mathrm{p}} / 3\right) \sim \widetilde{n}_{\mathrm{p}}\left(T_{\mathrm{b}}<m_{\mathrm{p}} / 3\right)=\widetilde{n}_{\mathrm{e}}\left(T_{\mathrm{e}}<m_{\mathrm{e}} / 3\right)$.

For the electron density, we neglect the slight offset due to the presence of Helium (that is neutrons) after the epoch of nucleosynthesis. Clearly also, the electron density is relative to free electrons only before recombination.

For a relativistic species $i$, including possibly the Dark Matter itself, it will turn out to be more convenient to use (e.g. in Appendix B.2, to relate various interaction rates) the quantity

$\widetilde{h_{i}}(T)=\frac{\phi_{i} a^{4}}{3 T_{0}}=625 \mathrm{~cm}^{-3} \frac{4 g^{\prime}{ }_{* i}(T)}{3}$,

rather than the comoving number density $\widetilde{n}_{i}$ of species $i$, to which however $\widetilde{h_{i}}$ is close.

\section{A.2.6. Reduced interaction rates}

As for the comoving number density, to remove the most obvious cosmological dependence of the interaction rates $\Gamma$ and leave only the temperature dependence related to the microphysics, we introduce "reduced" interaction rates:

$\widetilde{\Gamma}=\Gamma a^{3}$.

We define the time $t_{\mathrm{dec}(\mathrm{dm}-i)}$ and the corresponding scale-factor $a_{\mathrm{dec}(\mathrm{dm}-i)}$, related to the epoch where Dark Matter particles decouple from the species $i$. It is, by convention, taken to be the epoch where the interaction rate of the Dark Matter with the species $i$, namely $\Gamma_{\mathrm{dm}-i}$ is equal to the Hubble rate:

$\Gamma_{\mathrm{dm}-i}=H$.

The value of the reduced interaction rate $\widetilde{\Gamma}_{\mathrm{dm}-i}$ at the Dark Matter decoupling from the species $i$ will be denoted it $\widetilde{\Gamma}_{\mathrm{dec}(\mathrm{dm}-i)}$. It corresponds to

$\widetilde{\Gamma}_{\mathrm{dec}(\mathrm{dm}-i)}=\left.\Gamma_{\mathrm{dm}-i} a^{3}\right|_{a=a_{\mathrm{dec}(\mathrm{dm}-i)}}$.

We also need the total interaction rate of a species $i$, which includes the interactions with all species $j$ (including $i$ )

$\widetilde{\Gamma}_{i}=\sum_{j} \widetilde{\Gamma}_{i-j}$
The quantity $\widetilde{\Gamma}_{\operatorname{dec}(i)}$ will then denote the value of $\widetilde{\Gamma}_{i}$ at the time $t_{\operatorname{dec}(i)}$ and scale-factor $a_{\operatorname{dec}(i)}$ where species $i$ totally decouples (even with itself).

For generality, we denote by $\widetilde{\Gamma}_{\operatorname{dec}(x)}$ and $\Gamma_{\operatorname{dec}(x)}$ the reduced and normal interaction rate associated with $x$, where $x$ stands either for a particular coupling $i-j$ between the species $i$ and $j$ or simply for the full coupling of a species $i$ to the medium. The relation between $\widetilde{\Gamma}_{\operatorname{dec}(x)}$ and $\Gamma_{\operatorname{dec}(x)}$ may be written:

$\Gamma_{\operatorname{dec}(x)}=H_{\alpha}\left(\frac{\widetilde{\Gamma}_{\operatorname{dec}(x)}}{H_{\alpha}}\right)^{-\frac{1}{3 \alpha-1}}$.

This relation takes two different forms:

Radiation dominated era:

$\Gamma_{\operatorname{dec}(x)}=H_{\text {eq }}\left(\frac{\widetilde{\Gamma}_{\text {dec }(x)}}{\widetilde{H}_{\text {eq }}}\right)^{-2}$,

Matter dominated era:

$\Gamma_{\operatorname{dec}(x)}=H_{\mathrm{eq}}\left(\frac{\widetilde{\Gamma}_{\mathrm{dec}(x)}}{\widetilde{H}_{\mathrm{eq}}}\right)^{-1}$.

\section{A.3. Typical scales}

\section{A.3.1. Interaction rates}

The neutrino-electron and the photon-electron interaction rate play a central role in the determination of the damping induced by respectively the neutrinos and the photons.

Taking for the neutrino-electron cross-section (averaged over particles and antiparticles as well as over the three flavors)

$\sigma_{v-\mathrm{e}} c=4.02 \times 10^{-34} \mathrm{~cm}^{3} \mathrm{~s}^{-1} \frac{E_{v} E_{\mathrm{e}}}{(1 \mathrm{MeV})^{2}}$

we get, after statistical average

$\widetilde{\Gamma}_{v-\mathrm{e}}=\left\langle\sigma_{v-\mathrm{e}} v\right\rangle \widetilde{n}_{\mathrm{e}}$,

where $\widetilde{n}_{\mathrm{e}}$ is the total (relativistic) electron density including electrons and antielectrons. This rate varies as $\propto T^{2}$ because of the energy-dependence of the cross-section. At $v$-e decoupling (given by $\Gamma_{v-\mathrm{e}}=H$ ), this rate takes the value $\widetilde{\Gamma}_{\operatorname{dec}(v-\mathrm{e}) \text {. }}$

For non-relativistic electrons, up to recombination, the $\gamma-\mathrm{e}$ interaction rate is a constant as a function of time:

$\widetilde{\Gamma}_{\gamma-\mathrm{e}}=\widetilde{\Gamma}_{\mathrm{Th}}=\sigma_{\mathrm{Th}} c \widetilde{n}_{\mathrm{e}}$,

where $\sigma_{\mathrm{Th}}=\frac{8 \pi \alpha_{\mathrm{EM}}^{2}}{3 m_{\mathrm{e}}^{2}}$ is the Thomson cross-section.

Numerical values of these rates are given in Table A.2.

\section{A.3.2. Scale-factors (Table A.1)}

Several typical scale-factors may be defined. They are associated with the time-scales used for the calculation of the damping effects.

There first is $a_{\mathrm{nr}}$, the epoch at which the Dark Matter becomes non-relativistic, taken by convention to correspond to $T_{\mathrm{dm}}=m_{\mathrm{dm}} / 3$. 
Table A.1. Specific scale-factors.

\begin{tabular}{ll}
\hline \hline$a_{\mathrm{nr}}$ & $=\frac{3 T_{0}}{m_{\mathrm{dm}} \kappa_{\mathrm{dm}}\left(T_{\mathrm{nr}}\right)}$ \\
& $=7.06 \times 10^{-10}\left(\frac{m_{\mathrm{dm}} \kappa_{\mathrm{dm}}\left(T_{\mathrm{nr}}\right)}{1 \mathrm{MeV}}\right)^{-1}$ \\
\hline$a_{\mathrm{eq}(\gamma+v)}$ & $=\frac{\rho_{\gamma+v}\left(T_{0}\right)}{\rho_{\mathrm{m}}\left(T_{0}\right)}$ \\
& $=2.85 \times 10^{-4}\left(\frac{\Omega_{\mathrm{m}} h_{70}{ }^{2}}{0.3}\right)^{-1}$ \\
\hline$a_{\mathrm{eq}}$ & $=H_{\mathrm{r}}^{2} / H_{\mathrm{m}}^{2}$ \\
& $=g^{\prime}{ }_{*}\left(T_{\mathrm{eq}}\right) a_{\mathrm{eq}(\gamma+v)}$ \\
& $(\mathrm{species} i \mathrm{relativistic})$ \\
$a_{\mathrm{eq}(\mathrm{dm}-i)}(T)$ & $=\frac{p_{i} a^{4}}{\rho_{0 \mathrm{dm}}}$ \\
& $=\frac{4 g^{\prime}{ }_{* i}(T)}{3} \frac{\Omega_{\mathrm{m}}}{\Omega_{\mathrm{dm}}} a_{\mathrm{eq}(\gamma+v \text { ref })}$ \\
& $=3.41 \times 10^{-4} \frac{4 g^{\prime}{ }_{* i}(T)}{3}\left(\frac{\Omega_{\mathrm{dm}} h_{70}{ }^{2}}{0.25}\right)^{-1}$
\end{tabular}

NRFO scenario:

$\left(\phi_{\mathrm{dm}}\right.$ evaluated at an epoch where it is relativistic)

$$
\begin{aligned}
a_{\mathrm{eq}(\mathrm{dm})}(T) & =\frac{p_{\mathrm{dm}} a^{4}}{\rho_{0 \mathrm{dm}}} \\
& =\frac{4 g^{\prime}{ }_{* \mathrm{dm}}(T)}{3} \frac{\Omega_{\mathrm{m}}}{\Omega_{\mathrm{dm}}} a_{\mathrm{eq}(\gamma+v \text { ref })} \\
& =3.41 \times 10^{-4} \frac{4 g^{\prime}{ }_{* \mathrm{dm}}(T)}{3}\left(\frac{\Omega_{\mathrm{dm}} h_{70}{ }^{2}}{0.25}\right)^{-1} \\
a_{\mathrm{dec}(\mathrm{dm}-i)} & =\left(\frac{\widetilde{\Gamma}_{\mathrm{dec}(\mathrm{dm}-i)}}{H_{\alpha}}\right)^{\frac{\alpha}{3 \alpha-1}} \\
& =2.85 \times 10^{-4}\left(\frac{\widetilde{\Gamma}_{\mathrm{dec}(\mathrm{dm}-i)}}{5.96 \times 10^{-24} \mathrm{~s}^{-1}}\right){g^{\prime}}_{*}^{-\frac{1}{2}}\left(T_{\mathrm{dec}(\mathrm{dm})}\right)
\end{aligned}
$$

in the radiation era

$$
=2.85 \times 10^{-4}\left(\frac{\widetilde{\Gamma}_{\mathrm{dec}(\mathrm{dm}-i)}}{5.96 \times 10^{-24} \mathrm{~s}^{-1}}\right)^{2 / 3}\left(\frac{\Omega_{\mathrm{m}} h_{70}{ }^{2}}{0.3}\right)^{-\frac{1}{3}}
$$

in the matter era

\begin{tabular}{ll}
\hline$a_{\mathrm{dec}(v-\mathrm{e})}$ & $=\frac{\widetilde{\Gamma}_{\mathrm{dec}(v-\mathrm{e})}}{H_{\mathrm{r}}}$ \\
& $=1.33 \times 10^{-10}{g^{\prime}}^{-\frac{1}{6}} \kappa^{-\frac{2}{3}}$ \\
\hline$a_{\mathrm{rec}}$ & $=1 / 1090$ \\
\hline$a_{\mathrm{nl}}$ & $\sim 1 / 10 \quad$ (but left as a free parameter) \\
\hline
\end{tabular}

We are led to define the scale-factor $a_{\mathrm{eq}(\gamma+v \text { ref })}$ which characterizes the epoch of equality between matter and photon+neutrino energy-densities in our "reference" cosmology and $a_{\mathrm{eq}}$ the true epoch of matter-radiation equality in the general case, related to $H_{\mathrm{r}}$ and $H_{\mathrm{m}}$ already defined in Sect. A.2.2.

In order to get simpler expressions for the borderlines in the induced-damping case, we define also

$a_{\mathrm{eq}(\mathrm{dm}-i)}=\phi_{i} a^{4} / \rho_{0 \mathrm{dm}}$,
Table A.2. Specific reduced rates.

$$
\begin{aligned}
& \widetilde{H}_{\mathrm{nr}} \quad=1.48 \times 10^{-29} \mathrm{~s}^{-1}{g^{\prime}}_{*}^{\frac{1}{2}}\left(\frac{m_{\mathrm{dm}} \kappa_{\mathrm{dm}}}{1 \mathrm{MeV}}\right)^{-1} \\
& \text { in the radiation era } \\
& \widetilde{H}_{\mathrm{nr}} \quad=2.33 \times 10^{-32} \mathrm{~s}^{-1}\left(\frac{\Omega_{\mathrm{m}} h_{70}{ }^{2}}{0.3}\right)^{\frac{1}{2}}\left(\frac{m_{\mathrm{dm}} \kappa_{\mathrm{dm}}}{1 \mathrm{MeV}}\right)^{-\frac{3}{2}} \\
& \text { in the matter era } \\
& \widetilde{H}_{\mathrm{eq}} \quad=5.96 \times 10^{-24} \mathrm{~s}^{-1}{g^{\prime}}_{*}^{\frac{3}{2}}\left(T_{\mathrm{eq}}\right)\left(\frac{\Omega_{\mathrm{m}} h_{70}{ }^{2}}{0.3}\right)^{-1} . \\
& \widetilde{H}_{\mathrm{eq}(\mathrm{dm}-i)}=7.16 \times 10^{-24} \mathrm{~s}^{-1} \frac{4 g^{\prime}{ }_{* i}(T)}{3} g^{\prime_{*}^{2}}\left(\frac{\Omega_{\mathrm{dm}} h_{70}{ }^{2}}{0.25}\right)^{-1} \\
& \widetilde{\Gamma}_{\operatorname{dec}(v-\mathrm{e})}=2.80 \times 10^{-30} \mathrm{~s}^{-1} g_{*}^{\prime \frac{1}{3}} \kappa^{-\frac{2}{3}}, \\
& \widetilde{H}_{\mathrm{rec}} \quad=3.45 \times 10^{-23} \mathrm{~s}^{-1}\left(\frac{\Omega_{\mathrm{m}} h_{70}{ }^{2}}{0.3}\right)^{\frac{1}{2}}, \\
& \widetilde{\Gamma}_{\mathrm{Th}} \quad=\sigma_{\mathrm{Th}} c \widetilde{n}_{\mathrm{e}}=5.49 \times 10^{-21} \mathrm{~s}^{-1} \frac{\Omega_{\mathrm{b}} h_{70}{ }^{2}}{0.05}, \\
& \widetilde{H}_{\mathrm{nl}} \quad=3.93 \times 10^{-20} \mathrm{~s}^{-1}\left(\frac{a_{\mathrm{nl}}}{0.1}\right)^{\frac{3}{2}}\left(\frac{\Omega_{\mathrm{m}} h_{70}{ }^{2}}{0.3}\right)^{\frac{1}{2}}
\end{aligned}
$$

the ratio of the energy-density of a relativistic species $i$ extrapolated to the present-day to the present-day energy-density of the Dark Matter . If the Dark Matter is non-relativistic at $a_{\mathrm{eq}(\mathrm{dm}-i)}$, we have $\phi_{i} / \phi_{\mathrm{dm}}=a_{\mathrm{eq}(\mathrm{dm}-i)} / a$. So, $a_{\mathrm{eq}(\mathrm{dm}-i)}$ can be loosely interpreted -the true definition being in all cases the relation aboveas the epoch of equality between the energy-densities of the Dark Matter and species $i$. Its true interpretation is that it provides a constant value which measures the ratio of the energy density of a relativistic species to the present-day nonrelativistic Dark Matter energy-density. At times where the Dark Matter is relativistic, we can define the ratio

$a_{\mathrm{eq}(\mathrm{dm})}=\phi_{\mathrm{dm}} a^{4} / \rho_{0 \mathrm{dm}}$,

which we denote just by analogy with $a_{\mathrm{eq}(\mathrm{dm}-i)}$ as $a_{\mathrm{eq}(\mathrm{dm})}$. It is the ratio of the relativistic Dark Matter energy-density extrapolated to the present epoch to the actual (non-relativistic) energy-density. While, again, the true definition is the relation above, when evaluated at $a=a_{\text {nr }}$, we see this quantity to be closely related to the offset of the energy-density extrapolated from the relativistic side and the energy-density extrapolation fron the non-relativistic side, that is to the annihilation factor at this epoch. It is introduced to provide a measure of this annihilation factor, and is of interest only in the NRFO scenario.

The epoch of decoupling of Dark Matter with species $i$ is denoted $a_{\mathrm{dec}(\mathrm{dm}-\mathrm{i})}$. We have especially $a_{\mathrm{dec}(\mathrm{dm}-\nu)}$ and $a_{\mathrm{dec}(\mathrm{dm}-\gamma)}$, the epoch at which Dark Matter decouples from neutrinos and photons respectively. The epoch at which Dark Matter decouples from all species including itself corresponds to $a_{\mathrm{dec}(\mathrm{dm})}$. We 
Table A.3. Specific cross-sections.

$$
\begin{aligned}
& \hline s_{v}=4.48 \times 10^{-33} \mathrm{~cm}^{3} \mathrm{~s}^{-1} g^{\frac{1}{3}}\left(\frac{4 g^{\prime}{ }_{* v}(T)}{3}\right)^{-1} \kappa^{-\frac{2}{3}}, \\
& s_{\mathrm{rec}}=5.53 \times 10^{-26} \mathrm{~cm}^{3} \mathrm{~s}^{-1}\left(\frac{4 g^{\prime}{ }_{* \gamma}(T)}{3}\right)^{-1}\left(\frac{\Omega_{\mathrm{m}} h_{70}{ }^{2}}{0.3}\right)^{\frac{1}{2}}, \\
& s_{\mathrm{Th}}=1.99 \times 10^{-14} \mathrm{~cm}^{3} \mathrm{~s}^{-1} \\
& s_{\mathrm{eq}}=1.15 \times 10^{-26} \mathrm{~cm}^{3} \mathrm{~s}^{-1}\left(\frac{\Omega_{\mathrm{dm}} h_{70}{ }^{2}}{0.25}\right)^{-1}
\end{aligned}
$$

introduce in addition the scale-factor which defines the epoch of decoupling of neutrinos with electrons, namely $a_{\mathrm{dec}(v-\mathrm{e}) \text {. }}$.

For completeness, we recall that $a_{\text {rec }}$ is the scale-factor corresponding to recombination. Needless to say, its value is taken from the recent WMAP results. The epoch of the non-linear collapse, corresponding to $a_{\mathrm{nl}}$, will also be needed for strongly interacting Dark Matter particles.

The analytical and numerical expressions of all of these scale-factors are summarized in Table A.1. The Dark Matter properties typically enter these expression by means of the Dark Matter mass $m_{\mathrm{dm}}$ and interaction rate $\widetilde{\Gamma}_{\mathrm{dec}(\mathrm{dm}-i)}$ or $\widetilde{\Gamma}_{\mathrm{dec}(\mathrm{dm})}$.

\section{A.3.3. Typical Hubble rates (Table A.2)}

One can translate the above scale-factors into reduced Hubble rates $\widetilde{H}$ in order to define typical rates. The relation between $H$ and the scale-factor $a$ may be found in Sect. A.2.2. By convention, we define $\widetilde{H}_{\mathrm{eq}(\mathrm{dm}-i)}$ as $\widetilde{H}_{\mathrm{eq}(\mathrm{dm}-i)}=a_{\mathrm{eq}(\mathrm{dm}-i)} H_{\mathrm{r}}$. Together with the rates given in Sect. A.3.1, this sets the typical scales, displayed in Table A.2, to which the Hubble rate $\widetilde{H}$ and the reduced interaction rates $\widetilde{\Gamma}$ of Sect. A.2.6 are to be compared.

\section{A.3.4. Cross-section scales (Table A.3)}

Our results may also be displayed in terms of the Dark Matter $\langle\overline{\sigma v}\rangle$ cross-sections, more directly connected to particle physics. The latter are defined in terms of the interaction rate by:

$\widetilde{\Gamma}_{i j}=\langle\overline{\sigma v}\rangle_{i j} \widetilde{n}_{j}$

The values which set the scales of the $\langle\overline{\sigma v}\rangle$ cross-sections relevant to the limits we seek in this work (see e.g. the relations given in Table B.1 which are used in Appendix B.2) turn out to be for neutrinos

$s_{v}=\frac{\widetilde{\Gamma}_{\operatorname{dec}(v-\mathrm{e})}}{\widetilde{h}_{v}}$

while for photons we are led to use the values of the $\langle\overline{\sigma v}\rangle$ crosssections related to the epoch of recombination

$s_{\mathrm{rec}}=\frac{\widetilde{H}_{\mathrm{rec}}}{\widetilde{h}_{\gamma}}$,

or values of $\langle\overline{\sigma v}\rangle$ related to the Thomson cross-section

$s_{\mathrm{Th}}=\sigma_{\mathrm{Th}} c$.
Table B.1. Relation between $i-\mathrm{dm}$ and $\mathrm{dm}-i$ interaction rates and cross-sections for a relativistic species $i$.

$$
\begin{array}{ll}
\hline \hline a>a_{\mathrm{nr}} & \mathrm{URFO} \text { and NRFO } \\
& \frac{p_{\mathrm{dm}}}{p_{i}}=\frac{a}{a_{\mathrm{eq}(\mathrm{dm}-i)}} \\
& \widetilde{\Gamma}_{i-\mathrm{dm}}=\frac{a}{a_{\mathrm{eq}(\mathrm{dm}-i)}} \widetilde{\Gamma}_{\mathrm{dm}-i} . \\
& \kappa_{\mathrm{dm}}\langle\overline{\sigma v}\rangle_{i-\mathrm{dm}}=\frac{a}{a_{\mathrm{nr}}} \frac{\widetilde{\Gamma}_{\mathrm{dm}-i}}{\widetilde{h}_{i}} \\
\hline a<a_{\mathrm{nr}} & \mathrm{URFO} \\
& \frac{p_{\mathrm{dm}}}{\phi_{i}}=\frac{a_{\mathrm{nr}}}{a_{\mathrm{eq}(\mathrm{dm}-i)}} \\
& \widetilde{\Gamma}_{i-\mathrm{dm}}=\frac{a_{\mathrm{nr}}}{a_{\mathrm{eq}(\mathrm{dm}-i)}} \widetilde{\Gamma}_{\mathrm{dm}-i .} \\
& \kappa_{\mathrm{dm}}\langle\overline{\sigma v}\rangle_{i-\mathrm{dm}}=\frac{\widetilde{\Gamma}_{\mathrm{dm}-i}}{\widetilde{h}_{i}} \\
\hline a<a_{\mathrm{nr}} & \mathrm{NRFO} \\
& \frac{p_{\mathrm{dm}}}{\phi_{i}}=\frac{g_{* \mathrm{dm}}^{\prime}}{g^{\prime}{ }_{* i}} \\
& \widetilde{\Gamma}_{i-\mathrm{dm}}=\frac{g_{* \mathrm{dm}}^{\prime}}{g_{* i}^{\prime}} \widetilde{\Gamma}_{\mathrm{dm}-i} . \\
\kappa_{\mathrm{dm}}\langle\overline{\sigma v}\rangle_{i-\mathrm{dm}}=\frac{\widetilde{\Gamma}_{i-\mathrm{dm}}}{\widetilde{h}_{\mathrm{dm}}}=\frac{\widetilde{\Gamma}_{\mathrm{dm}-i}}{\widetilde{h}_{i}} \\
\hline
\end{array}
$$

Finally, we will also be led to use, for any relativistic species $i$,

$s_{\mathrm{eq}}=\frac{\widetilde{H}_{\mathrm{eq}(\mathrm{dm}-i)}}{\widetilde{\not h}_{i}}$.

While the definition of $s_{\text {eq }}$ is always the above relation, it may be (somewhat loosely) interpreted as the $\langle\overline{\sigma v}\rangle$ cross-section needed for the Dark Matter to decouple from species $i$ just at the epoch of equality of the energy-densities $\phi_{i}$ and $\rho_{0 \mathrm{dm}} / a^{3}$. Note that $s_{\text {eq }}$, which may be readily evaluated as $s_{\text {eq }}=\frac{H_{\mathrm{r}}}{\rho_{0 \mathrm{dm}} / 3 T_{0}}$ by means of the expressions of $\widetilde{H}_{\mathrm{eq}(\mathrm{dm}-i)}$ (Table A.2) and $\widetilde{h}_{i}$ (Sect. A.2.5), turns out to be independent of the relativistic species $i$ considered.

These typical cross-sections are given in Table A.3.

\section{Appendix B: Density evolution and relations among interaction rates}

\section{B.1. Density evolution}

In case the Dark Matter is non relativistic at the epoch of reference, and if the number of particles is conserved till nowadays, one has

$\widetilde{n}_{\mathrm{dm}}=\frac{\Omega_{\mathrm{dm}} \rho_{0 c}}{m_{\mathrm{dm}}}$.

In the NRFO scenario, strictly speaking, this relation is valid only fr $a>a_{\mathrm{fo}} \sim 7 a_{\mathrm{nr}}$. In the URFO scenario, it is valid even for relativistic Dark Matter. 


\section{Boehm and R. Schaeffer: Dark Matter damping lengths, Online Material p 7}

In the NRFO scenario, for relativistic Dark Matter, the number density may be written ${ }^{12}$

$\widetilde{n}_{\mathrm{dm}}=\widetilde{h}_{\mathrm{dm}} \kappa_{\mathrm{dm}}(T)$,

where $\widetilde{\not h}$ is the density introduced in Sect. A.2.5. As expected, $\widetilde{n}_{\mathrm{dm}}$ does not depend on the Dark Matter particles' mass. It is independent of time except for possible variations of the factor $\kappa_{\mathrm{dm}}(T)$ or those due to the coefficient $g_{* \mathrm{dm}}$ contained in $\widetilde{h}_{\mathrm{dm}}$. The densities on the relativistic and the non-relativistic side in the NRFO scenario are related by

$\widetilde{n}_{\mathrm{dm}}\left(T<T_{\mathrm{nr}}\right)=\widetilde{h}_{\mathrm{dm}}\left(T_{\mathrm{nr}}\right) \frac{\kappa_{\mathrm{dm}}\left(T_{\mathrm{nr}}\right) a_{\mathrm{nr}}}{a_{\mathrm{eq}(\mathrm{dm})}}$.

Although the Dark Matter density does not depend on which species $i$ is present in the system, a quite useful relation relating the non-relativistic Dark Matter density to the density $\widetilde{h}_{i}$ (see Sect. A.2.5) of a relativistic species $i$ is

$\widetilde{n}_{\mathrm{dm}}\left(T<T_{\mathrm{nr}}\right)=\widetilde{\breve{h}_{i}} \frac{\kappa_{\mathrm{dm}}\left(T_{\mathrm{nr}}\right) a_{\mathrm{nr}}}{a_{\mathrm{eq}(\mathrm{dm}-i)}}$.

\section{B.2. Expression of the $\Gamma_{i-\mathrm{dm}}$ rate and the $\langle\overline{\sigma v}\rangle_{i-\mathrm{dm}}$ cross-section in terms of $\Gamma_{\mathrm{dm}-i}$}

The relation (23) between the rates $\widetilde{\Gamma}_{i-\mathrm{dm}}$ and $\widetilde{\Gamma}_{\mathrm{dm}-i}$ is governed by the ratio of the energy densities $\phi_{\mathrm{dm}} / \phi_{i}$ relative to these two species:

$\widetilde{\Gamma}_{i-\mathrm{dm}}=\frac{\phi_{\mathrm{dm}}}{\phi_{i}} \widetilde{\Gamma}_{\mathrm{dm}-i}$.

The expressions of $\widetilde{\Gamma}_{i-\mathrm{dm}}$ may be transformed into expressions of $\langle\overline{\sigma v}\rangle_{i-\mathrm{dm}}$ by means of

$\langle\overline{\sigma v}\rangle_{i-\mathrm{dm}}=\frac{\widetilde{\Gamma}_{i-\mathrm{dm}}}{\widetilde{n}_{\mathrm{dm}}}$.

To study the correspondence between the interaction rates and cross-sections, we need to establish the evolution of the number density of Dark Matter particles, that may or may not be the same before and after the non-relativistic transition.

We seek a relation between $\widetilde{\Gamma}_{i-\mathrm{dm}}$ and $\widetilde{\Gamma}_{\mathrm{dm}-i}$. In the special case it is evaluated at an epoch $a$ such as the Dark Matter particle number is conserved afterwards, the latter can be written in a more explicit form than the general relation (23). To this purpose, we evaluate the ratio $\phi_{i} / \phi_{\mathrm{dm}}$, specifically under this assumption, for relativistic and non-relativistic Dark Matter particles.

\section{B.2.1. Relativistic species $i$}

This is the case we explicitly need to consider in the present paper.

\footnotetext{
12 The exact relation differs by a numerical coefficient very close to unity. We use the above approximation because it joins continuously to the non-relativistic expression at $a=a_{\mathrm{nr}}$. This turns out to be accurate enough for our purpose and yields substantially simpler practical calculations (it avoids in particular to distinguish fermionic and bosonic Dark Matter).
}

In case the Dark Matter particles are already nonrelativistic, that is for $a>a_{\mathrm{nr}}$, for both the URFO and the NRFO scenario, we have $\frac{\phi_{\mathrm{dm}}}{\phi_{i}}=\frac{a}{a_{\mathrm{eq}(\mathrm{dm}-\mathrm{i})}}$.

If the Dark Matter particles are still relativistic, on the other hand, for $a<a_{\mathrm{nr}}$, we get in the URFO scenario ${ }^{13} \phi_{\mathrm{dm}} \sim$ $\rho_{0 \mathrm{dm}} a_{\mathrm{nr}} / a^{4}$, and thus $\frac{\phi_{\mathrm{dm}}}{\phi_{i}}=\frac{a_{\mathrm{nr}}}{a_{\mathrm{eq}(\mathrm{dm}-i)}}$.

In the NRFO scenario, on the other hand, when $a$ correspond to an epoch where the Dark Matter still is relativistic, since the annihilation is still to come, the Dark Matter radiation density may be expected to be of the order of the density of any other relativistic species. More precisely, for $a<a_{\mathrm{nr}}$, one has $\frac{\phi_{\mathrm{dm}}}{\phi_{i}}=\frac{g_{* \mathrm{dm}}^{\prime}}{g_{* i}^{\prime}}$. The ratio $g_{* i}^{\prime} / g^{\prime}{ }_{* \mathrm{dm}}$, except in very exotic models, is expected (taking into account that we are in the NRFO scenario) to be of order unity.

These relation, which will be needed throughout the whole paper, and the inferred relation among interaction rates and cross-sections are recalled in Table B.1.

\section{B.2.2. Nonrelativistic species $i$}

Similar expression may be established in this case. They will turn out not to be needed in the practical applications. We give them here (Table B.2) for the sake of completeness: in the remainder of the paper, only the expressions for relativistic species $i$ will be given. To this purpose, it turns out to be convenient, rather than $a_{\mathrm{eq}(\mathrm{dm}-i)}$, which is a quantity adapted to relativistic species $i$, to use another ratio suited to nonrelativistic species $i$ :

$f_{\mathrm{dm} / i}=\frac{\rho_{0 \mathrm{dm}}}{\rho_{i} a^{3}}$.

In case the number of particles $i$ is conserved, one has obviously $f_{\mathrm{dm} / i}=\frac{\Omega_{\mathrm{odm}}}{\Omega_{0 i}}$.

\section{B.3. The non-relativistic transition in the NRFO scenario}

In the NRFO scenario, there is a sudden decrease of the Dark Matter particle number when the latter becomes nonrelativistic. We approximate the transition around $a=a_{\mathrm{nr}}$ (and more precisely between $a=a_{\mathrm{nr}}$ and $a=a_{\mathrm{fo}} \sim 7 a_{\mathrm{nr}}$ ) by an abrupt transition at $a=a_{\mathrm{nr}}$ where some physical quantities (such as for instance the density) switch abruptly from a "relativistic" value to a "non-relativistic" value. This results in a great simplification in displaying our results, and is of no harm, provided the associated discontinuities at $a=a_{\mathrm{nr}}$ are properly evaluated.

It is readily seen from Sect. B.1 that in the NRFO scenario, there is a drop of the density by a factor

$\frac{\left.\widetilde{n}_{\mathrm{dm}}\left(T_{\mathrm{nr}}\right)\right|_{\mathrm{nr}}}{\left.\widetilde{n}_{\mathrm{dm}}\left(T_{\mathrm{nr}}\right)\right|_{\mathrm{r}}}=\frac{a_{\mathrm{nr}}}{a_{\mathrm{eq}(\mathrm{dm})}}$.

13 The exact relation differs by a numerical coefficient. The density $\widetilde{n}_{\mathrm{dm}}$ in Sect. B.1 (see the footnote there) is related to this expression of the energy-density by taking $3 T_{\mathrm{dm}}$ as the average energy of a particle independently of the statistics. This insures consistency with our choice to set the non-relativistic transition at $m_{\mathrm{dm}}=3 T_{\mathrm{dm}}$. 
Table B.2. Relation between $i-\mathrm{dm}$ and $\mathrm{dm}-i$ interaction rates and cross-sections for a nonrelativistic species $i$.

\begin{tabular}{ll}
\hline \hline & $\langle\overline{\sigma v}\rangle_{\mathrm{dm}-i}=\frac{\widetilde{\Gamma}_{\mathrm{dm}-i}}{\widetilde{n}_{i}}$ \\
\hline$a>a_{\mathrm{nr}}$ & URFO \& NRFO \\
& $\frac{p_{\mathrm{dm}}}{p_{i}}=f_{\mathrm{dm} / i}$ \\
& $\widetilde{\Gamma}_{i-\mathrm{dm}}=f_{\mathrm{dm} / i} \widetilde{\Gamma}_{\mathrm{dm}-i}$ \\
& $\langle\overline{\sigma v}\rangle_{i-\mathrm{dm}}=\frac{m_{\mathrm{dm}}}{m_{i}} \frac{\widetilde{\Gamma}_{\mathrm{dm}-i}}{\widetilde{n}_{i}}=\frac{m_{\mathrm{dm}}}{m_{i}}\langle\overline{\sigma v}\rangle_{\mathrm{dm}-i}$ \\
\hline$a<a_{\mathrm{nr}}$ & $\mathrm{URFO}$ \\
& $\frac{p_{\mathrm{dm}}}{\phi_{i}}=f_{\mathrm{dm} / i} \frac{a_{\mathrm{nr}}}{a}$ \\
& $\widetilde{\Gamma}_{i-\mathrm{dm}}=f_{\mathrm{dm} / i} \frac{a_{\mathrm{nr}}}{a} \widetilde{\Gamma}_{\mathrm{dm}-i}$ \\
& $\langle\overline{\sigma v}\rangle_{i-\mathrm{dm}}=\frac{a_{\mathrm{nr}}}{a} \frac{m_{\mathrm{dm}}}{m_{i}} \frac{\widetilde{\Gamma}_{\mathrm{dm}-i}}{\widetilde{n}_{i}}=\frac{a_{\mathrm{nr}}}{a} \frac{m_{\mathrm{dm}}}{m_{i}}\langle\overline{\sigma v}\rangle_{\mathrm{dm}-i}$ \\
\hline$a<a_{\mathrm{nr}}$ & $\mathrm{NRFO}$ \\
& $\frac{p_{\mathrm{dm}}}{\phi_{i}}=f_{\mathrm{dm} / i} \frac{a_{\mathrm{eq}(\mathrm{dm})}}{a}$ \\
& $\widetilde{\Gamma}_{i-\mathrm{dm}}=f_{\mathrm{dm} / i} \frac{a_{\mathrm{eq}(\mathrm{dm})}}{a} \widetilde{\Gamma}_{\mathrm{dm}-i}$ \\
& $\langle\overline{\sigma v}\rangle_{i-\mathrm{dm}}=\frac{a_{\mathrm{nr}}}{a} \frac{m_{\mathrm{dm}}}{m_{i}} \frac{\widetilde{\Gamma}_{\mathrm{dm}-i}}{\widetilde{n}_{i}}=\frac{a_{\mathrm{nr}}}{a} \frac{m_{\mathrm{dm}}}{m_{i}}\langle\overline{\sigma v}\rangle_{\mathrm{dm}-i}$
\end{tabular}

This ratio represents the annihilation factor, due to the decay of the Dark Matter particles between $a_{\mathrm{nr}}$ and $a_{\mathrm{fo}}$, needed to insure cosmological consistency. To represent really annihilation it is obviously required to be smaller than unity. This makes sense only in Regions I, II, III $\left(a_{\mathrm{eq}(\gamma+v \text { ref })}>a_{\mathrm{nr}}\right)$ since we expect $a_{\text {eq(dm) }} \sim a_{\text {eq }(\gamma+v \text { ref })}$.

The ratio of the non-relativistic value $\left.\phi_{\mathrm{dm}}\left(a_{\mathrm{nr}}\right)\right|_{\mathrm{nr}}$ to the relativistic value $\left.\phi_{\mathrm{dm}}\left(a_{\mathrm{nr}}\right)\right|_{\mathrm{r}}$ at $a_{\mathrm{nr}}$ is also, from our previous estimates (Sect. B.2) of the ratio $\phi_{i} / \phi_{\mathrm{dm}}$

$\frac{\left.\phi_{\mathrm{dm}}\left(T_{\mathrm{nr}}\right)\right|_{\mathrm{nr}}}{\left.\phi_{\mathrm{dm}}\left(T_{\mathrm{nr}}\right)\right|_{\mathrm{r}}}=\frac{a_{\mathrm{nr}}}{a_{\mathrm{eq}(\mathrm{dm})}}$

The question which has been treated in Sect. B.2 is, given the interaction rate $\widetilde{\Gamma}_{\mathrm{dm}-i}$, taken at a fixed epoch, what is the expression of the rate $\widetilde{\Gamma}_{i-\mathrm{dm}}$ and the cross-section $\langle\overline{\sigma v}\rangle_{i-\mathrm{dm}}$, taken at the same epoch. We give explicitly here the discontinuities of the latter around $a=a_{\mathrm{nr}}$.

The rate $\widetilde{\Gamma}_{\mathrm{dm}-i}$ is obviously continuous around $a_{\mathrm{nr}}$. From the basic relation (23), we then readily see that

$\frac{\left.\widetilde{\Gamma}_{i-\mathrm{dm}}\left(T_{\mathrm{nr}}\right)\right|_{\mathrm{nr}}}{\left.\widetilde{\Gamma}_{i-\mathrm{dm}}\left(T_{\mathrm{nr}}\right)\right|_{\mathrm{r}}}=\frac{a_{\mathrm{nr}}}{a_{\mathrm{eq}(\mathrm{dm})}}$.

It bears the same discontinuity than the densities. From its definition, $\langle\overline{\sigma v}\rangle_{i-\mathrm{dm}}=\widetilde{\Gamma}_{i-\mathrm{dm}} \widetilde{n}_{\mathrm{dm}}$ is the ratio of two discontinuous quantities, but remains exactly continuous, as it should be, despite the approximations we have made. This is possible only thanks to the care we have taken in evaluating the approximate expressions of the energy-densities, and was by no means granted.

\section{Appendix C: Classification of the Dark Matter candidates}

As explained in Sect. 3, the calculation of the damping scales is separated into several contributions, the self-damping and free-streaming, neutrino and photon induced-damping. We treat each of these effects separately in order to display the different constraints in a more appropriate way. The damping lengths turn out to depend on the Dark Matter particles' mass and on an appropriate interaction rate. Their values thus may be displayed in a two-parameter space, respectively $\left[m_{\mathrm{dm}}, \widetilde{\Gamma}_{\mathrm{dec}(\mathrm{dm})}\right],\left[m_{\mathrm{dm}}, \widetilde{\Gamma}_{\mathrm{dec}(\mathrm{dm}-v)}\right],\left[m_{\mathrm{dm}}, \widetilde{\Gamma}_{\mathrm{dec}(\mathrm{dm}-\gamma)}\right]$ for the selfdamping and free-streaming, the neutrino induced-damping and photon induced-damping. The analytical expression of the damping takes different forms in the various "regions" of the relevant parameter space, as is seen in Sects. 4.1, 5.1 and 6.1, respectively. Here we give the borderlines of these regions.

\section{C.1. Dark Matter classification from self-damping and free-streaming}

The borderlines of the regions defined in Sect. 4.1 within which the damping lengths take different forms are given by the conditions

$a_{\mathrm{eq}(\gamma+v)}=a_{\mathrm{nr}}$,

$a_{\mathrm{dec}(\mathrm{dm})}=a_{\mathrm{eq}(\gamma+\gamma)}$,

$a_{\mathrm{dec}(\mathrm{dm})}=a_{\mathrm{nr}}$.

They cross at one single point, and define six regions, labelled from I to VI.

The discussion in terms of $a_{\mathrm{eq}(\gamma+\gamma)}$ is useful because this scale-factor remains defined (and relevant) even in regions IV, $\mathrm{V}$ and VI (although, in this case, it bears no relation with the epoch of equality which occurs at $a=a_{\mathrm{nr}}$ in these regions).

The numerical values of the above scale-factors are given in Appendix A.3.2. They imply the bordelines given explicitely in Table C.1.

\section{C.2. Borderlines of the Regions in the neutrino parameter space}

The damping length due to neutrino induced-damping is to be evaluated at Dark Matter-neutrino decoupling. All constraints on the interaction rates thus are obtained at this time. All relations given in this section hence are understood as being writ-

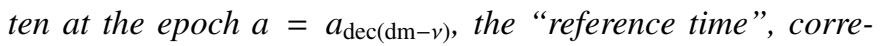
sponding to the decoupling of the Dark Matter with neutrinos.

Most important in this case are the interaction rates. They determine whether $v-\mathrm{e}$ or $v-\mathrm{dm}$ scattering or simply neutrino free-streaming is responsible for the damping, as discussed in Sect. 5.1. Depending on these rates, there are three regions in the $\left[m_{\mathrm{dm}}, \widetilde{\Gamma}_{\mathrm{dec}(\mathrm{dm}-v)}\right]$ plane, namely $\mathrm{A}, \mathrm{B}$ and $\mathrm{C}$ in which the induced-damping scale results from different physical processes:

- Region A: $\Gamma_{v-\mathrm{e}} \geq \Gamma_{v-\mathrm{dm}}, \Gamma_{\mathrm{dm}-v} \leq \Gamma_{v} \sim \Gamma_{v-\mathrm{e}}$, 
C. Boehm and R. Schaeffer: Dark Matter damping lengths, Online Material p 9

Table C.1. Explicit borderlines for self-damping and free-streaming.

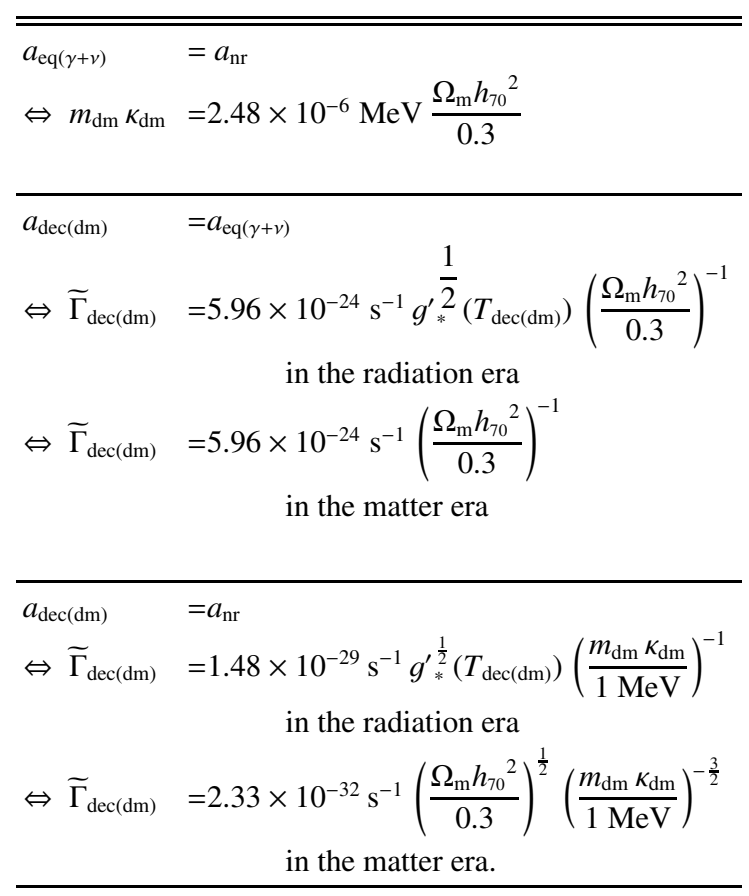

Table C.2. Borderlines for $\mathrm{dm}-v$ induced-damping.

\begin{tabular}{ccc}
\hline \hline A-B borderline & \\
$\widetilde{\Gamma}_{\mathrm{dm}-v} \leq \widetilde{\Gamma}_{v-\mathrm{dm}}$ & $\widetilde{\Gamma}_{v-\mathrm{dm}}=\widetilde{\Gamma}_{v-\mathrm{e}}$ \\
& A-C borderline & \\
$\widetilde{\Gamma}_{\mathrm{dm}-v} \leq \widetilde{\Gamma}_{v-\mathrm{dm}}$ & $\widetilde{\Gamma}_{\mathrm{dm}-v}=\widetilde{\Gamma}_{v-\mathrm{e}}$ \\
& & \\
& B-C borderline & \\
$\widetilde{\Gamma}_{v-\mathrm{e}}<\widetilde{\Gamma}_{v-\mathrm{dm}}$ & $\widetilde{\Gamma}_{\mathrm{dm}-v}=\widetilde{\Gamma}_{v-\mathrm{dm}}$ \\
and (NRFO): & $\widetilde{\Gamma}_{v-\mathrm{e}}<\widetilde{\Gamma}_{\mathrm{dm}-v}<\widetilde{H}_{\mathrm{eq}(\mathrm{dm}-v)}$ & $\widetilde{\Gamma}_{\mathrm{dm}-v}=\widetilde{H}_{\mathrm{nr}}$ \\
\hline
\end{tabular}

- Region B: $\Gamma_{v-\mathrm{e}}<\Gamma_{v-\mathrm{dm}}, \Gamma_{\mathrm{dm}-v} \leq \Gamma_{v} \sim \Gamma_{\nu-\mathrm{dm}}$,

- Region C: $\Gamma_{\mathrm{dm}-v}>\Gamma_{v}$.

The borderlines A-B, A-C, and B-C which separate the three regions (Table C.2) may be directly inferred from these definitions.

It is however of interest to express these relations in terms of $m_{\mathrm{dm}}$ and $\widetilde{\Gamma}_{\mathrm{dec}(\mathrm{dm}-v)}$. They may also be translated also in terms of $m_{\mathrm{dm}}$ and the cross-sections $\langle\overline{\sigma v}\rangle_{v-\mathrm{dm}}$. We will do this in the following two subsections (separating the URFO and the NRFO scenario) by using the relation between $\widetilde{\Gamma}_{v-\mathrm{dm}}$ and $\widetilde{\Gamma}_{\mathrm{dm}-v}$ established in Appendix B.2.
Table C.3. Explicit borderlines in the URFO dm- $v$ scenario.

$$
\begin{aligned}
& \begin{array}{ll}
\hline \hline \text { A-B } & \widetilde{\Gamma}_{\mathrm{dm}-v} \leq \widetilde{\Gamma}_{v-\mathrm{dm}} \quad \widetilde{\Gamma}_{v-\mathrm{dm}}=\widetilde{\Gamma}_{v-\mathrm{e}}
\end{array} \\
& a_{\mathrm{eq}(\mathrm{dm}-v)} \leq a_{\mathrm{nr}} \\
& \widetilde{\Gamma}_{\mathrm{dm}-v}=\left(\frac{a_{\mathrm{eq}(\mathrm{dm}-v)}}{a_{\mathrm{nr}}}\right)^{\frac{1}{3}} \widetilde{\Gamma}_{\mathrm{dec}(v-\mathrm{e})} \\
& \widetilde{\Gamma}_{v-d m}=\left(\frac{a_{\mathrm{eq}(\mathrm{dm}-v)}}{a_{\mathrm{nr}}}\right)^{-\frac{2}{3}} \widetilde{\Gamma}_{\mathrm{dec}(v-\mathrm{e})} \\
& \frac{\kappa_{\mathrm{dm}}\langle\overline{\sigma v}\rangle_{v-d m}=\left(\frac{a_{\mathrm{eq}(\mathrm{dm}-v)}}{a_{\mathrm{nr}}}\right)^{\frac{1}{3}} s_{v}}{\mathrm{~A}-\mathrm{C} \quad \widetilde{\Gamma}_{\mathrm{dm}-v}>\widetilde{\Gamma}_{v-\mathrm{dm}} \quad \widetilde{\Gamma}_{\mathrm{dm}-v}=\widetilde{\Gamma}_{v-\mathrm{e}}} \\
& a_{\mathrm{eq}(\mathrm{dm}-v)}>a_{\mathrm{nr}} \\
& \widetilde{\Gamma}_{\mathrm{dm}-v}=\widetilde{\Gamma}_{\mathrm{dec}(v-\mathrm{e})} \\
& a_{\mathrm{dec}(\mathrm{dm}-v)}>a_{\mathrm{nr}} \\
& \widetilde{\Gamma}_{v-\mathrm{dm}}=\frac{a_{\mathrm{dec}(v-\mathrm{e})}}{a_{\mathrm{eq}(\mathrm{dm}-v)}} \widetilde{\Gamma}_{\mathrm{dec}(v-\mathrm{e})} \\
& \kappa_{\mathrm{dm}}\langle\overline{\sigma v}\rangle_{v-\mathrm{dm}}=\frac{a_{\mathrm{dec}(v-\mathrm{e})}}{a_{\mathrm{nr}}} s_{v} \\
& \begin{aligned}
a_{\mathrm{dec}(\mathrm{dm}-v)} & <a_{\mathrm{nr}} \\
& \widetilde{\Gamma}_{v-\mathrm{dm}}=\frac{a_{\mathrm{nr}}}{a_{\mathrm{eq}(\mathrm{dm}-v)}} \widetilde{\Gamma}_{\mathrm{dec}(v-\mathrm{e})}
\end{aligned} \\
& \begin{array}{ll}
\kappa_{\mathrm{dm}}\langle\overline{\sigma v}\rangle_{v-\mathrm{dm}}=s_{v} \\
\hline \mathrm{B}-\mathrm{C} \quad \widetilde{\Gamma}_{v-\mathrm{e}}<\widetilde{\Gamma}_{v-\mathrm{dm}} \quad \widetilde{\Gamma}_{\mathrm{dm}-v}=\widetilde{\Gamma}_{v-\mathrm{dm}}
\end{array} \\
& a_{\mathrm{dec}(v-\mathrm{e})}<a_{\mathrm{dec}(\mathrm{dm}-v)} \\
& a_{\mathrm{dec}(\mathrm{dm}-v)}>a_{\mathrm{nr}} \\
& \widetilde{\Gamma}_{\mathrm{dm}-v}=\widetilde{H}_{\mathrm{eq}(\mathrm{dm}-v)} \\
& \widetilde{\Gamma}_{v-\mathrm{dm}}=\widetilde{H}_{\mathrm{eq}(\mathrm{dm}-v)} \\
& \kappa_{\mathrm{dm}}\langle\overline{\sigma v}\rangle_{v-\mathrm{dm}}=\frac{a_{\mathrm{eq}(\mathrm{dm}-v)}}{a_{\mathrm{nr}}} s_{\mathrm{eq}} \\
& a_{\mathrm{dec}(\mathrm{dm}-v)}<a_{\mathrm{nr}} \\
& a_{\mathrm{eq}(\mathrm{dm}-v)}=a_{\mathrm{nr}}
\end{aligned}
$$

\section{C.2.1. URFO $\mathrm{dm}-v$ scenario (Table C.3)}

\section{C.2.2. NRFO dm-v scenario (Table C.4)}

Here, we only consider the cases for which $a_{\mathrm{eq}(\mathrm{dm}-v)}>a_{\mathrm{nr}}$. The case corresponding to $a_{\mathrm{eq}(\mathrm{dm}-v)}<a_{\mathrm{nr}}$, is very unnatural as discussed in Sect. 4.2.6.

A-B borderline. For $a_{\mathrm{dec}(\mathrm{dm}-v)}>a_{\mathrm{nr}}$, as in the URFO scenario, we have $a_{\mathrm{eq}(\mathrm{dm}-v)}>a_{\mathrm{nr}}$, which is the case that we have decided to not consider explicitely.

A-C borderline. For $a_{\mathrm{dec}(\mathrm{dm}-v)}<a_{\mathrm{nr}}$, i.e. relativistic Dark Matter, one would have $\widetilde{\Gamma}_{\mathrm{dm}-v}=\widetilde{\Gamma}_{v-\mathrm{dm}}$, which is not possible in Region C. 
Table C.4. Explicit borderlines for the NRFO $\mathrm{dm}-v$ scenario (Excluding the case $a_{\mathrm{nr}}>a_{\mathrm{eq}(\mathrm{dm}-v)}$, as discussed in the text).

\begin{tabular}{|c|c|}
\hline A-B & $\widetilde{\Gamma}_{\mathrm{dm}-v} \leq \widetilde{\Gamma}_{v-\mathrm{dm}} \quad \widetilde{\Gamma}_{v-\mathrm{dm}}=\widetilde{\Gamma}_{v-\mathrm{e}}$ \\
\hline & $\begin{array}{l}a_{\mathrm{dec}(\mathrm{dm}-v)} \leq a_{\mathrm{nr}} \\
\widetilde{\Gamma}_{\mathrm{dm}-v}=\left(\frac{g^{\prime}{ }_{* \mathrm{dm}}}{g^{\prime}{ }_{* v}}\right)^{\frac{1}{3}} \widetilde{\Gamma}_{\mathrm{dec}(v-\mathrm{e})} \\
\widetilde{\Gamma}_{v-\mathrm{dm}}=\frac{g_{* \mathrm{dm}}^{\prime}}{g_{* v}^{\prime}} \widetilde{\Gamma}_{\mathrm{dm}-v} \\
\kappa_{\mathrm{dm}}\langle\overline{\sigma v}\rangle_{\nu-\mathrm{dm}}=\left(\frac{g_{* \mathrm{dm}}^{\prime}}{g^{\prime}{ }^{\frac{1}{3}} s_{v}}\right.\end{array}$ \\
\hline $\mathrm{A}-\mathrm{C}$ & $\begin{array}{l}\widetilde{\Gamma}_{\mathrm{dm}-v}>\widetilde{\Gamma}_{v-\mathrm{dm}} \quad \widetilde{\Gamma}_{\mathrm{dm}-v}=\widetilde{\Gamma}_{v-\mathrm{e}} \\
a_{\mathrm{dec}(\mathrm{dm}-v)}>a_{\mathrm{nr}} \\
\widetilde{\Gamma}_{\mathrm{dm}-v}=\widetilde{\Gamma}_{\mathrm{dec}(v-\mathrm{e})} \\
\widetilde{\Gamma}_{v-\mathrm{dm}}=\frac{a_{\mathrm{dec}(v-\mathrm{e})}}{a_{\mathrm{eq}(\mathrm{dm}-v)}} \widetilde{\Gamma}_{\mathrm{dec}(v-\mathrm{e})} \\
\kappa_{\mathrm{dm}}\langle\overline{\sigma v}\rangle_{v-\mathrm{dm}}=\frac{a_{\mathrm{dec}(v-\mathrm{e})}}{a_{\mathrm{nr}}} s_{v}\end{array}$ \\
\hline $\mathrm{B}-\mathrm{C}$ & $\begin{array}{l}\widetilde{\Gamma}_{v-\mathrm{e}}<\widetilde{\Gamma}_{v-\mathrm{dm}} \quad \widetilde{\Gamma}_{\mathrm{dm}-v}=\widetilde{\Gamma}_{v-\mathrm{dm}} \\
a_{\mathrm{dec}(v-\mathrm{e})}<a_{\mathrm{dec}(\mathrm{dm}-v)} \\
\widetilde{\Gamma}_{\mathrm{dm}-v}=\widetilde{H}_{\mathrm{eq}(\mathrm{dm}-v)} \\
\widetilde{\Gamma}_{v-\mathrm{dm}}=\widetilde{\Gamma}_{\mathrm{dm}-v} \\
\kappa_{\mathrm{dm}}\langle\overline{\sigma v}\rangle_{v-\mathrm{dm}}=\frac{a_{\mathrm{eq}(\mathrm{dm}-v)}}{a_{\mathrm{nr}}} s_{\mathrm{eq}}\end{array}$ \\
\hline $\mathrm{B}-\mathrm{C}$ & $\begin{array}{l}\widetilde{\Gamma}_{v-\mathrm{e}}<\widetilde{\Gamma}_{\mathrm{dm}-v}<\widetilde{H}_{\mathrm{eq}(\mathrm{dm}-v)} \quad \widetilde{\Gamma}_{\mathrm{dm}-v}=\widetilde{H}_{\mathrm{nr}} \\
a_{\mathrm{dec}(v-\mathrm{e})}<a_{\mathrm{dec}(\mathrm{dm}-v)}=a_{\mathrm{nr}}<a_{\mathrm{eq}(\mathrm{dm}-v)}\end{array}$ \\
\hline B side: & $\begin{array}{l}\left.\text { (where } \widetilde{\Gamma}_{v-\mathrm{e}}<\widetilde{\Gamma}_{v} \sim \widetilde{\Gamma}_{v-\mathrm{dm}} \sim \widetilde{\Gamma}_{\mathrm{dm}-v}\right) \\
\widetilde{\Gamma}_{v-\mathrm{dm}}=\frac{g_{* \mathrm{dm}}^{\prime}}{g_{* v}^{\prime}} \widetilde{H}_{\mathrm{nr}}\end{array}$ \\
\hline $\mathrm{C}$ side: & $\begin{array}{l}\left(\text { where } \widetilde{\Gamma}_{v-\mathrm{e}}<\widetilde{\Gamma}_{v} \sim \widetilde{\Gamma}_{v-\mathrm{dm}}<\widetilde{\Gamma}_{\mathrm{dm}-v}\right) \\
\widetilde{\Gamma}_{v-\mathrm{dm}}=\frac{a_{\mathrm{nr}}}{a_{\mathrm{eq}(\mathrm{dm}-v)}} \widetilde{H}_{\mathrm{nr}}\end{array}$ \\
\hline $\mathrm{B}$ and $\mathrm{C}$ & $\kappa_{\mathrm{dm}}\langle\overline{\sigma v}\rangle_{v-\mathrm{dm}}=\frac{a_{\mathrm{nr}}}{a_{\mathrm{eq}(\mathrm{dm}-v)}} s_{\mathrm{eq}}$ \\
\hline
\end{tabular}

B-C borderline. For $a_{\mathrm{dec}(\mathrm{dm}-v)}=a_{\mathrm{nr}}$, that is for $\widetilde{\Gamma}_{\mathrm{dm}-v}=\widetilde{H}_{\mathrm{nr}}$, there is a borderline where $\widetilde{\Gamma}_{v-\mathrm{dm}}$ is discontinuous. On the relativistic side, we are in Region B, with $\widetilde{\Gamma}_{\mathrm{dm}-v}=\widetilde{H}_{\mathrm{nr}} \sim \widetilde{\Gamma}_{v-\mathrm{dm}}$. On the non-relativistic side, we are in Region $\mathrm{C}$ with $\widetilde{\Gamma}_{\mathrm{dm}-v}=$ $\widetilde{H}_{\mathrm{nr}}>\widetilde{\Gamma}_{v-\mathrm{dm}}=\frac{a_{\mathrm{nr}}}{a_{\mathrm{eq}(\mathrm{dm}-v)}} \widetilde{H}_{\mathrm{nr}}$. This however can only be the case for $a_{\mathrm{dec}(v-\mathrm{e})}<a_{\mathrm{nr}}$.

\section{C.3. Borderlines of the Regions in the photon parameter space}

There is now one single borderline since there are only two regions $\mathrm{A}$ and $\mathrm{B}$ of interest to compute the photon induceddamping scale.
Table C.5. Borderlines for the $\mathrm{dm}-\gamma$ scenario.

$$
\begin{aligned}
& \begin{array}{lll}
\hline \hline \text { A-B } & \widetilde{\Gamma}_{\mathrm{dm}-\gamma}<\widetilde{H}_{\mathrm{rec}} & \widetilde{\Gamma}_{\gamma-\mathrm{dm}}=\widetilde{\Gamma}_{\gamma-\mathrm{e}}
\end{array} \\
& \begin{array}{ll}
\mathrm{A}-\mathrm{B} & \widetilde{\Gamma}_{\mathrm{dm}-\gamma}=\widetilde{H}_{\mathrm{rec}} \\
\hline
\end{array}
\end{aligned}
$$

Table C.6. Explicit borderlines for the URFO dm $-\gamma$ scenario.

$$
\begin{array}{ll}
\hline \hline \text { A-B } & \widetilde{\Gamma}_{\mathrm{dm}-\gamma}<\widetilde{H}_{\mathrm{rec}} \quad \widetilde{\Gamma}_{\gamma-\mathrm{dm}}=\widetilde{\Gamma}_{\gamma-\mathrm{e}} \\
& a_{\mathrm{dec}(\mathrm{dm}-\gamma)}<a_{\mathrm{rec}} \\
& \widetilde{\Gamma}_{\mathrm{dm}-\gamma}=\frac{a_{\mathrm{eq}(\mathrm{dm}-\gamma)} \widetilde{\Gamma}_{\mathrm{Th}}}{a_{\mathrm{nr}}} \\
& \widetilde{\Gamma}_{\gamma-\mathrm{dm}}=\widetilde{\Gamma}_{\mathrm{Th}} \\
& \kappa_{\mathrm{dm}}\langle\overline{\sigma v}\rangle_{\gamma-\mathrm{dm}}=\frac{a_{\mathrm{eq}(\mathrm{dm}-\gamma)}}{a_{\mathrm{nr}}} \frac{\widetilde{n}_{\mathrm{e}}}{\widetilde{h}_{\gamma}} s_{\mathrm{Th}} \\
\hline \text { A-B } & \widetilde{\Gamma}_{\mathrm{dm}-\gamma}=\widetilde{H}_{\mathrm{rec}} \\
& a_{\mathrm{dec}(\mathrm{dm}-\gamma)}=a_{\mathrm{rec}} \\
a_{\mathrm{dec}(\mathrm{dm}-\gamma)}>a_{\mathrm{nr}} & \widetilde{\Gamma}_{\gamma-\mathrm{dm}}=\frac{a_{\mathrm{dec}(\mathrm{dm}-\gamma)}}{a_{\mathrm{eq}(\mathrm{dm}-\gamma)} \widetilde{H}_{\mathrm{rec}}} \\
& \kappa_{\mathrm{dm}}\langle\overline{\sigma v}\rangle_{\gamma-\mathrm{dm}}=\frac{a_{\mathrm{dec}(\mathrm{dm}-\gamma)}}{a_{\mathrm{nr}}} s_{\mathrm{rec}} \\
& \widetilde{\Gamma}_{\gamma-\mathrm{dm}}=\frac{a_{\mathrm{nr}}}{a_{\mathrm{eq}(\mathrm{dm}-\gamma)}} \widetilde{H}_{\mathrm{rec}} \\
& \kappa_{\mathrm{dm}}\langle\overline{\sigma v}\rangle_{\gamma-\mathrm{dm}}=s_{\mathrm{rec}} \\
\hline
\end{array}
$$

All relations given in this section are understood as being

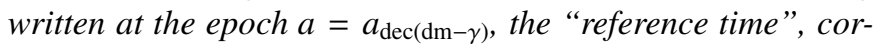
responding to the decoupling of the Dark Matter with photons.

Provided $\widetilde{\Gamma}_{\mathrm{dm}-\gamma}<\widetilde{H}_{\text {rec }}$, the first borderline (Table C.5) corresponds to

$\widetilde{\Gamma}_{\gamma-\mathrm{dm}}=\widetilde{\Gamma}_{\gamma-\mathrm{e}} \equiv \widetilde{\Gamma}_{\mathrm{Th}}$.

This may be rewritten in terms of $\widetilde{\Gamma}_{\mathrm{dm}-\gamma}$ by means of the relations of Sect. B.2. Relativistic Dark Matter, in the URFO scenario leads to a borderline, given in Table C.6, where we have $\widetilde{\Gamma}_{\mathrm{dm}-\gamma}=\frac{a_{\mathrm{eq}(\mathrm{dm}-\gamma)}}{a_{\mathrm{nr}}} \widetilde{\Gamma}_{\mathrm{Th}}$. For all sensible values of $a_{\mathrm{nr}}$ namely $a_{\mathrm{nr}}<$ $a_{\mathrm{eq}(\mathrm{dm}-\gamma} \widetilde{\Gamma}_{\mathrm{Th}} / H_{\mathrm{eq}(\mathrm{dm}-\gamma)}$, it corresponds also to Dark Matter which decouples in the matter dominated era. It is also readily seen that $\widetilde{\Gamma}_{\mathrm{dm}-\gamma}<\widetilde{H}_{\text {rec }}$ implies $a_{\mathrm{nr}}>a_{\mathrm{eq}(\mathrm{dm}-\gamma)} \widetilde{\Gamma}_{\mathrm{Th}} / H_{\text {rec }}$. Note that the limit is reached at a value $a_{\mathrm{nr}}=a_{\mathrm{eq}(\mathrm{dm}-\gamma)} \widetilde{\Gamma}_{\mathrm{Th}} / H_{\mathrm{rec}}>a_{\mathrm{eq}(\mathrm{dm}-\gamma)}$.

The second borderline (see Tables C.6 and C.7), in the URFO as well as the NRFO case, corresponds to

$\widetilde{\Gamma}_{\mathrm{dm}-\gamma}=\widetilde{H}_{\mathrm{rec}}$

and is relevant for the smaller values of $a_{\mathrm{nr}}$, namely $a_{\mathrm{nr}}<$ $a_{\mathrm{eq}(\mathrm{dm}-\gamma)} \widetilde{\Gamma}_{\mathrm{Th}} / H_{\mathrm{rec}}$, that is for the larger masses $m_{\mathrm{dm}}$.

\section{Appendix D: Damping limits}

We give in this section the analytical form of the constraint one obtains by writing that the various damping lengths have to be 
Table C.7. Explicit borderlines for the NRFO $\mathrm{dm}-\gamma$ scenario (Evaluated only for $a_{\mathrm{nr}}<a_{\mathrm{eq}(\mathrm{dm}-\gamma)}$ as discussed in the text).

\begin{tabular}{ll}
\hline \hline A-B & $\widetilde{\Gamma}_{\mathrm{dm}-\gamma}=\widetilde{H}_{\mathrm{rec}}$ \\
& $a_{\mathrm{dec}(\mathrm{dm}-\gamma)}=a_{\mathrm{rec}}$ \\
& $\widetilde{\Gamma}_{\gamma-\mathrm{dm}}=\frac{a_{\mathrm{dec}(\mathrm{dm}-\gamma)}}{a_{\mathrm{eq}(\mathrm{dm}-\gamma)} \widetilde{H}_{\mathrm{rec}}}$ \\
& $\kappa_{\mathrm{dm}}\langle\overline{\sigma v}\rangle_{\gamma-\mathrm{dm}}=\frac{a_{\mathrm{dec}(\mathrm{dm}-\gamma)}}{a_{\mathrm{nr}}} s_{\mathrm{rec}}$ \\
\hline
\end{tabular}

smaller than a given a priori chosen limit $l_{\text {struct }}$. Note that in each case, the parameter space is constrained by the relevant damping limit and the condition to be in a given "Region" in which the expression used for the damping scale is valid.

\section{D.1. Self-damping}

The damping scales in this case are given as a fraction of the horizon at decoupling $\pi c t_{\mathrm{r}} a_{\mathrm{dec}(\mathrm{dm})}$, or $\pi c t_{\mathrm{m}} a_{\mathrm{dec}(\mathrm{dm})}^{\frac{1}{2}}$. Noting that $t_{\mathrm{m}}$ differs from $t_{\mathrm{r}}$ basically by a factor $a_{\mathrm{eq}}^{\frac{1}{2}}$ (Sect. A.2.1), one sees all damping lengths can be expressed as a fraction of

$l_{\text {eq }}=\pi c t_{\mathrm{r}} a_{\mathrm{eq}}=207 \mathrm{Mpc} g_{*}^{\prime \frac{1}{2}}\left(\frac{\Omega_{\mathrm{m}} h_{70}{ }^{2}}{0.3}\right)^{-1}$.

The analytic expression of the damping scales and the associated limits on the interaction rates and the $\langle\overline{\sigma v}\rangle$ cross-sections are given in Table D.1 for the six Regions which cover the parameter space. The free-streaming damping scale has been calculated via the approximate expression (40), to be directly comparable to the expression of the self-damping length, estimated using a similar approximation (32) and to be related to the mass scale by Eq. (41). In Regions I, II and III where our limits are set, we give for completeness - within brackets - also the expression of the free-streaming scale when it is by convention taken to be given by Eq. (39). All relations given in this section are understood as being written at the epoch $a=a_{\mathrm{dec}(\mathrm{dm})}$, the "reference time", corresponding to the decoupling of Dark Matter with every species including itself.

\section{D.2. Neutrino induced-damping}

The typical scale in this case is the damping scale of the neutrinos at the time they decouple from the electrons

$l_{\operatorname{dec}(v-\mathrm{e})}=\pi c t_{\mathrm{r}} r_{v} \frac{\widetilde{\Gamma}_{\mathrm{dec}(v-\mathrm{e})}}{H_{\mathrm{r}}}=97.1 \mathrm{pc} r_{v} \kappa^{-\frac{2}{3}}{g^{\prime}}^{-\frac{2}{3}}$.

For Region A, the damping is too small to be relevant to structure formation. For Region B, only the case $a_{\mathrm{dec}(\mathrm{dm}-v)}<$ $a_{\mathrm{eq}(\mathrm{dm}-v)}$ has been considered explicitly: for $a_{\mathrm{dec}(\mathrm{dm}-v)}>$ $a_{\text {eq(dm- }-v)}$, the damping is too large. Needless to say, in the latter case, too, the damping may be readily evaluated by means of the same methods.

Analytical expression of the damping scales and limits on interaction rates and $\langle\overline{\sigma v}\rangle$ are given in Tables D.2-D.6.
Table D.1. Self-damping limits.

$$
\begin{aligned}
& \begin{array}{ll}
\hline \hline \text { Region I } & =l_{\mathrm{eq}} \frac{a_{\mathrm{nr}}}{a_{\mathrm{eq}}} \\
l_{\mathrm{fs}} & \left.=l_{\mathrm{eq}} \frac{a_{\mathrm{nr}}}{a_{\mathrm{eq}}} \frac{5+2 \ln \left(a_{\mathrm{eq}} / a_{\mathrm{nr}}\right)}{\pi}\right] \\
l_{\mathrm{fs}} & =l_{\mathrm{eq}} r_{\mathrm{dm}} \frac{a_{\mathrm{dec}(\mathrm{dm})}}{a_{\mathrm{eq}}} \\
l_{\mathrm{sd}} & <a_{\mathrm{eq}} \frac{l_{\mathrm{struct}}}{l_{\mathrm{eq}}} \\
a_{\mathrm{nr}} &
\end{array} \\
& l_{\mathrm{fs}}=\frac{l_{\mathrm{sd}}}{r_{\mathrm{rm}}} \quad=l_{\mathrm{eq}}\left(\frac{a_{\mathrm{nr}} a_{\mathrm{dec}(\mathrm{dm})}}{a_{\mathrm{eq}}^{2}}\right)^{\frac{1}{2}} \\
& \begin{array}{lll}
{\left[l_{\mathrm{fs}}\right.} & \left.=l_{\mathrm{eq}}\left(\frac{a_{\mathrm{nr}} a_{\mathrm{dec}(\mathrm{dm})}}{a_{\mathrm{eq}}^{2}}\right)^{\frac{1}{2}} \frac{3+2 \ln \left(a_{\mathrm{eq}} / a_{\mathrm{dec}}\right)}{\pi}\right] \\
a_{\mathrm{nr}} & <\frac{a_{\mathrm{eq}}^{2}}{a_{\mathrm{dec}(\mathrm{dm})}}\left(\frac{l_{\mathrm{struct}}}{l_{\mathrm{eq}}}\right)^{2}
\end{array} \\
& l_{\mathrm{fs}}=\frac{l_{\mathrm{sd}}}{r_{\mathrm{rm}}} \quad=l_{\mathrm{eq}}\left(\frac{a_{\mathrm{nr}}}{a_{\mathrm{eq}}}\right)^{\frac{1}{2}} \\
& {\left[l_{\mathrm{fs}} \quad=l_{\mathrm{eq}}\left(\frac{a_{\mathrm{nr}}}{a_{\mathrm{eq}}}\right)^{\frac{1}{2}} \frac{3}{\pi}\right]} \\
& a_{\mathrm{nr}} \quad<a_{\mathrm{eq}}\left(\frac{l_{\text {struct }}}{l_{\mathrm{eq}}}\right)^{2}
\end{aligned}
$$

Region III'

$\begin{array}{ll}\frac{l_{\mathrm{sd}}}{r_{\mathrm{rm}}} & =l_{\mathrm{eq}}\left(\frac{\widetilde{H}_{\mathrm{nl}}}{\widetilde{\Gamma}_{\mathrm{dm}}}\right)^{\frac{1}{2}}\left(\frac{a_{\mathrm{nr}}}{a_{\mathrm{eq}}}\right)^{1 / 2}, \\ a_{\mathrm{nr}} & <\frac{\widetilde{\Gamma}_{\mathrm{dm}}}{\widetilde{H}_{\mathrm{nl}}} a_{\mathrm{eq}}\left(\frac{l_{\mathrm{struct}}}{l_{\mathrm{eq}}}\right)^{2}\end{array}$

Region IV

$\begin{array}{ll}l_{\mathrm{fs}} & =l_{\mathrm{eq}} \frac{a_{\mathrm{nr}}}{a_{\mathrm{eq}}} \\ l_{\mathrm{sd}} & =l_{\mathrm{eq}} r_{\mathrm{dm}} \frac{a_{\mathrm{dec}(\mathrm{dm})}}{a_{\mathrm{eq}}} \\ a_{\mathrm{nr}} & <a_{\mathrm{eq}} \frac{l_{\mathrm{struct}}}{l_{\mathrm{eq}}}\end{array}$

Region V

$\begin{array}{ll}l_{\mathrm{fs}} & =l_{\mathrm{eq}} \frac{a_{\mathrm{nr}}}{a_{\mathrm{eq}}} \\ l_{\mathrm{sd}} & =l_{\mathrm{eq}} r_{\mathrm{dm}} \frac{a_{\mathrm{dec}(\mathrm{dm})}}{a_{\mathrm{eq}}} \\ a_{\mathrm{nr}} & <a_{\mathrm{eq}} \frac{l_{\mathrm{struct}}}{l_{\mathrm{eq}}}\end{array}$

Region VI

$$
\begin{array}{ll}
l_{\mathrm{fs}}=\frac{l_{\mathrm{sd}}}{r_{\mathrm{rm}}} & =l_{\mathrm{eq}}\left(\frac{a_{\mathrm{nr}}}{a_{\mathrm{eq}}}\right)^{\frac{1}{2}} \\
a_{\mathrm{nr}} & <a_{\mathrm{eq}}\left(\frac{l_{\mathrm{struct}}}{l_{\mathrm{eq}}}\right)^{2}
\end{array}
$$

Region $\mathrm{VI}^{\prime}$

$$
\begin{array}{ll}
\frac{l_{\mathrm{sd}}}{r_{\mathrm{rm}}} & =l_{\mathrm{eq}}\left(\frac{\widetilde{H}_{\mathrm{nl}}}{\widetilde{\Gamma}_{\mathrm{dm}}}\right)^{\frac{1}{2}}\left(\frac{a_{\mathrm{nr}}}{a_{\mathrm{eq}}}\right)^{1 / 2}, \\
a_{\mathrm{nr}} & <\frac{\widetilde{\Gamma}_{\mathrm{dm}}}{\widetilde{H}_{\mathrm{nl}}} a_{\mathrm{eq}}\left(\frac{l_{\mathrm{struct}}}{l_{\mathrm{eq}}}\right)^{2}
\end{array}
$$

All relations given in this section are understood as being writ- 
Table D.2. Region A (dm- $v$ scenario).

$$
\overline{l_{v \mathrm{~d}} \quad=l_{\operatorname{dec}(v-\mathrm{e})}\left(\widetilde{\Gamma}_{\mathrm{dm}-v} / \widetilde{\Gamma}_{\mathrm{dec}(v-\mathrm{e})}\right)^{\frac{3}{2}}}
$$

Table D.3. Region B (URFO dm - v scenario).

$$
\begin{aligned}
\hline & =l_{\mathrm{dec}(v-\mathrm{e})}\left(\frac{a_{\mathrm{eq}(\mathrm{dm}-v)}}{a_{\mathrm{nr}}}\right)^{\frac{1}{2}} \frac{\widetilde{\Gamma}_{\mathrm{dm}-v}}{\widetilde{\Gamma}_{\mathrm{dec}(v-\mathrm{e})}} \\
\widetilde{\Gamma}_{\mathrm{dm}-v} & <\widetilde{\Gamma}_{\mathrm{dec}(v-\mathrm{e})}\left(\frac{a_{\mathrm{nr}}}{a_{\mathrm{eq}(\mathrm{dm}-v)}}\right)^{\frac{1}{2}} \frac{l_{\mathrm{struct}}}{l_{\mathrm{dec}(v-\mathrm{e})}} \\
\kappa_{\mathrm{dm}}\langle\overline{\sigma v}\rangle_{v-\mathrm{dm}} & =\frac{\widetilde{\Gamma}_{\mathrm{dm}-v}}{h_{v}} \\
& <s_{v}\left(\frac{a_{\mathrm{nr}}}{a_{\mathrm{eq}(\mathrm{dm}-v)}}\right)^{\frac{1}{2}} \frac{l_{\mathrm{struct}}}{l_{\mathrm{dec}(v-\mathrm{e})}}
\end{aligned}
$$

Table D.4. Region B (NRFO dm- $v$ scenario).

$$
\begin{array}{ll}
\hline l_{v \mathrm{~d}} & =l_{\operatorname{dec}(v-\mathrm{e})}\left(\frac{g^{\prime}{ }_{* v}}{g^{\prime}{ }_{* \mathrm{dm}}}\right)^{\frac{1}{2}} \frac{\widetilde{\Gamma}_{\mathrm{dm}-v}}{\widetilde{\Gamma}_{\mathrm{dec}(v-\mathrm{e})}} \\
\widetilde{\Gamma}_{\mathrm{dm}-v} & <\widetilde{\Gamma}_{\operatorname{dec}(v-\mathrm{e})}\left(\frac{g^{\prime}{ }_{* \mathrm{dm}}}{g^{\prime}{ }_{* v}}\right)^{\frac{1}{2}} \frac{l_{\mathrm{struct}}}{l_{\operatorname{dec}(v-\mathrm{e})}} \\
\kappa_{\mathrm{dm}}\langle\overline{\sigma v}\rangle_{v-\mathrm{dm}} & =\frac{\widetilde{\Gamma}_{\mathrm{dm}-v}}{h_{\nu}}<s_{v}\left(\frac{g^{\prime}{ }_{* \mathrm{dm}}}{g^{\prime}{ }_{* v}}\right)^{\frac{1}{2}} \frac{l_{\text {struct }}}{l_{\mathrm{dec}(v-\mathrm{e})}}
\end{array}
$$

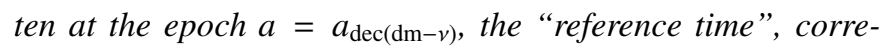
sponding to the decoupling of the Dark Matter with photons.

\section{D.3. Photon induced-damping}

A unit to characterize the damping scale in this case may be chosen as the horizon scale at equality, weighted by a slightly time-dependent factor $r_{\gamma}$, to be taken at the proper time:

$$
\begin{aligned}
l_{\mathrm{eq}(\mathrm{dm}-\gamma)} & =\pi c t_{\mathrm{r}} r_{\gamma} a_{\mathrm{eq}(\mathrm{dm}-\gamma)} \\
& =249 \mathrm{Mpc} r_{\gamma}{g^{\prime}}^{-\frac{1}{2}} \frac{4{g^{\prime}}_{* i}(T)}{3}\left(\frac{\Omega_{\mathrm{dm}} h_{70}{ }^{2}}{0.25}\right)^{-1} .
\end{aligned}
$$

In Region A, rather than the URFO and NRFO scenarios which turn out here to yield the same damping limits, it turns out to be useful to distinguish two cases, depending on whether Dark Matter decoupling occurs in the radiation dominated era $\left(a_{\mathrm{dec}(\mathrm{dm}-\gamma)}<a_{\mathrm{eq}}\right)$, or in the matter dominated era $\left(a_{\mathrm{dec}(\mathrm{dm}-\gamma)}>\right.$ $\left.a_{\text {eq }}\right)$. We do not give the damping lengths when Dark Matter decouples from the photons in the matter dominated era since they are always prohibitive as compared to our requirements.

Analytical expression of the damping scales and limits on interaction rates and $\langle\overline{\sigma v}\rangle$ are given in Table D.7 for decou-
Table D.5. Region C (URFO dm $-v$ scenario).

$$
\begin{array}{ll}
\hline \hline l_{v \mathrm{~d}} & =l_{\mathrm{dec}(v-\mathrm{e})} \frac{\widetilde{\Gamma}_{\mathrm{dm}-v}}{\widetilde{\Gamma}_{\mathrm{dec}(v-\mathrm{e})}} \\
\widetilde{\Gamma}_{\mathrm{dm}-v} & <\widetilde{\Gamma}_{\mathrm{dec}(v-\mathrm{e})} \frac{l_{\mathrm{struct}}}{l_{\mathrm{dec}(v-\mathrm{e})}} \\
a_{\mathrm{dec}(\mathrm{dm}-v)}>a_{\mathrm{nr}} & \\
\kappa_{\mathrm{dm}}\langle\overline{\sigma v}\rangle_{v-\mathrm{dm}} & =\frac{a_{\mathrm{dec}(\mathrm{dm}-v)}}{a_{\mathrm{nr}}} \frac{\widetilde{\Gamma}_{\mathrm{dm}-v}}{h_{v}} \\
& <s_{v} \frac{a_{\mathrm{dec}(v-\mathrm{e})}}{a_{\mathrm{nr}}}\left(\frac{l_{\mathrm{struct}}}{l_{\mathrm{dec}(v-\mathrm{e})}}\right)^{2} \\
a_{\mathrm{dec}(\mathrm{dm}-v)}<a_{\mathrm{nr}} & \\
\kappa_{\mathrm{dm}}\langle\overline{\sigma v}\rangle_{v-\mathrm{dm}} & =\frac{\widetilde{\Gamma}_{\mathrm{dm}-v}}{h_{v}} \\
& <s_{v} \frac{l_{\mathrm{struct}}}{l_{\operatorname{dec}(v-\mathrm{e})}}
\end{array}
$$

Table D.6. Region C (NRFO dm $-v$ scenario).

\begin{tabular}{ll}
\hline \hline$l_{v \mathrm{~d}}$ & $=l_{\mathrm{dec}(v-\mathrm{e})} \frac{\widetilde{\Gamma}_{\mathrm{dm}-v}}{\widetilde{\Gamma}_{\mathrm{dec}(v-\mathrm{e})}}$ \\
$\widetilde{\Gamma}_{\mathrm{dm}-v}$ & $<\widetilde{\Gamma}_{\mathrm{dec}(v-\mathrm{e})} \frac{l_{\mathrm{struct}}}{l_{\operatorname{dec}(v-\mathrm{e})}}$ \\
$a_{\mathrm{dec}(\mathrm{dm}-v)}>a_{\mathrm{nr}}$ & $=\frac{a_{\mathrm{dec}(\mathrm{dm}-v)} \frac{\widetilde{\Gamma}_{\mathrm{dm}-v}}{a_{\mathrm{nr}}}}{\kappa_{\mathrm{dm}}\langle\overline{\sigma v}\rangle_{v-\mathrm{dm}}}$ \\
& $<s_{v} \frac{a_{\mathrm{dec}(v-\mathrm{e})}}{a_{\mathrm{nr}}}\left(\frac{l_{\mathrm{struct}}}{l_{\mathrm{dec}(v-\mathrm{e})}}\right)^{2}$ \\
$a_{\mathrm{dec}(\mathrm{dm}-v)}<a_{\mathrm{nr}}$ & \\
does not exist in this case & \\
\hline
\end{tabular}

pling in the radiation dominated era. They are obtained from the expression of the damping length given in Sect. 6.2 by means of the relations given in Table B.1 and the definitions of Appendix A. All relations given in this section are understood as being written at the epoch $a=a_{\mathrm{dec}(\mathrm{dm}-\gamma)}$, the "reference time", corresponding to the decoupling of the Dark Matter with photons. 
Table D.7. Region A, radiation dominated (URFO and NRFO dm $-\gamma$ scenario).

$$
\begin{array}{ll}
\hline & =l_{\text {eq }(\mathrm{dm}-\gamma)}\left(\frac{\widetilde{H}_{\mathrm{eq}(\mathrm{dm}-\gamma)}}{\widetilde{\Gamma}_{\mathrm{Th}}}\right)^{\frac{1}{2}}\left(\frac{\widetilde{\Gamma}_{\mathrm{dm}-\gamma}}{\widetilde{H}_{\mathrm{eq}(\mathrm{dm}-\gamma)}}\right)^{\frac{3}{2}} \\
\widetilde{\Gamma}_{\mathrm{dm}-\gamma} & <\widetilde{H}_{\mathrm{eq}(\mathrm{dm}-\gamma)}\left(\frac{\widetilde{H}_{\mathrm{eq}(\mathrm{dm}-\gamma)}}{\widetilde{\Gamma}_{\mathrm{Th}}}\right)^{-\frac{1}{3}}\left(\frac{l_{\mathrm{struct}}}{l_{\mathrm{eq}(\mathrm{dm}-\gamma)}}\right)^{\frac{2}{3}}
\end{array}
$$

$$
\begin{aligned}
a_{\mathrm{dec}(\mathrm{dm}-\gamma)} & >a_{\mathrm{nr}} \\
\kappa_{\mathrm{dm}}\langle\overline{\sigma v}\rangle_{\gamma-\mathrm{dm}} & =\frac{a_{\mathrm{dec}(\mathrm{dm}-\gamma)}}{a_{\mathrm{nr}}} \frac{\widetilde{\Gamma}_{\mathrm{dm}-\gamma}}{\not h_{\gamma}} \\
& <s_{\mathrm{eq}}\left(\frac{\widetilde{H}_{\mathrm{eq}(\mathrm{dm}-\gamma)}}{\widetilde{\Gamma}_{\mathrm{Th}}}\right)^{-\frac{2}{3}} \frac{a_{\mathrm{eq}(\mathrm{dm}-\gamma)}}{a_{\mathrm{nr}}}\left(\frac{l_{\mathrm{struct}}}{l_{\mathrm{eq}(\mathrm{dm}-\gamma)}}\right)^{\frac{4}{3}}
\end{aligned}
$$

$$
\begin{aligned}
a_{\mathrm{dec}(\mathrm{dm}-\gamma)} & <a_{\mathrm{nr}} \\
\kappa_{\mathrm{dm}}\langle\overline{\sigma v}\rangle_{\gamma-\mathrm{dm}} & =\frac{\widetilde{\Gamma}_{\mathrm{dm}-\gamma}}{\not h \gamma} \\
& <s_{\mathrm{eq}}\left(\frac{\widetilde{H}_{\mathrm{eq}(\mathrm{dm}-\gamma)}}{\widetilde{\Gamma}_{\mathrm{Th}}}\right)^{-\frac{1}{3}}\left(\frac{l_{\mathrm{struct}}}{l_{\mathrm{eq}(\mathrm{dm}-\gamma)}}\right)^{\frac{2}{3}}
\end{aligned}
$$

